JOURNAL OF THE

AMERICAN MATHEMATICAL SOCIETY

Volume 23, Number 3, July 2010, Pages 853-908

S 0894-0347(10)00664-8

Article electronically published on February 8, 2010

\title{
UNIQUENESS OF ENHANCEMENT FOR TRIANGULATED CATEGORIES
}

\author{
VALERY A. LUNTS AND DMITRI O. ORLOV
}

\section{Contents}

Introduction 853

1. DG categories, quasi-functors, and quotients of DG categories 856

2. Enhancements of triangulated categories and formulation of main results 864

3. Preliminary lemmas and propositions $\quad 867$

4. Preliminary constructions 873

5. Proof of Theorem 2.7 875

6. Proof of Theorem 2.8

7. Applications to commutative and noncommutative geometry 883

8. Bounded derived categories of coherent sheaves 887

9. Strong uniqueness and fully faithful functors $\quad 894$

Appendix A. Small U-cocomplete categories $\quad 901$

Appendix B. Proof of Proposition 9.6 905

Acknowledgments $\quad 907$

References $\quad 907$

\section{INTRODUCTION}

Triangulated categories were invented about 50 years ago as a convenient tool to do homological algebra. Yet it has been known for some time now that the notion of a triangulated category is not satisfactory: morphisms between objects in such a category are usually given by cohomology groups of certain complexes, and you forget too much by passing to cohomology. The problem is that the cone of a morphism is not functorial in triangulated categories. Let us give a couple of "frustrating" examples.

Given a triangulated category $\mathcal{T}$ we can consider the category $\mathcal{T}^{\vee}$ of all cohomological functors from $\mathcal{T}$ to the category of abelian groups. We "know" that $\mathcal{T}^{\vee}$

Received by the editors September 5, 2009 and, in revised form, December 14, 2009.

2010 Mathematics Subject Classification. Primary 14F05, 18E30.

Key words and phrases. Triangulated categories, DG categories, derived categories of sheaves.

The first author was partially supported by the NSA grant H98230-05-1-0050.

The second author was partially supported by grant RFFI 08-01-00297 and grant NSh1987.2008.1.

(C)2010 American Mathematical Society Reverts to public domain 28 years from publication 
should also be a triangulated category, which one might call the dual of $\mathcal{T}$. However one cannot prove that $\mathcal{T}^{\vee}$ is indeed triangulated.

Another example is the operation of the tensor product of two triangulated categories (which should also be a triangulated category) that cannot be performed without an extra data BLL].

Thus we like to consider a triangulated category $\mathcal{T}$ together with an enhancement $\mathcal{B}$, which has the same objects as $\mathcal{T}$ and the set of morphisms between two objects in $\mathcal{B}$ is a complex. One recovers morphisms in $\mathcal{T}$ by taking the cohomology $H^{0}$ of the corresponding morphism complex in $\mathcal{B}$. Thus $\mathcal{B}$ is a DG category and $\mathcal{T}$ is its homotopy category $H^{0}(\mathcal{B})$. The notion of a triangulated category lifts to the DG world [BK]: one has pretriangulated DG categories, in which the cone of a morphism is functorial!

Let $\mathcal{T}$ be a triangulated category. An enhancement of $\mathcal{T}$ is a pair $(\mathcal{B}, \varepsilon)$, where $\mathcal{B}$ is a pretriangulated DG category and $\varepsilon: H^{0}(\mathcal{B}) \stackrel{\sim}{\longrightarrow} \mathcal{T}$ is an equivalence of triangulated categories. There are questions of existence and uniqueness of enhancement for a given triangulated category.

The category $\mathcal{T}$ has a unique enhancement if it has one and for any two enhancements $(\mathcal{B}, \varepsilon)$ and $\left(\mathcal{B}^{\prime}, \varepsilon^{\prime}\right)$ of $\mathcal{T}$ the DG categories $\mathcal{B}$ and $\mathcal{B}^{\prime}$ are quasi-equivalent; i.e., there exists a quasi-functor $\phi: \mathcal{B} \longrightarrow \mathcal{B}^{\prime}$ which induces an equivalence $H^{0}(\phi)$ : $H^{0}(\mathcal{B}) \stackrel{\sim}{\longrightarrow} H^{0}\left(\mathcal{B}^{\prime}\right)$. In this case the enhancements $(\mathcal{B}, \varepsilon)$ and $\left(\mathcal{B}^{\prime}, \varepsilon^{\prime}\right)$ are called equivalent.

Enhancements $(\mathcal{B}, \varepsilon)$ and $\left(\mathcal{B}^{\prime}, \varepsilon^{\prime}\right)$ of $\mathcal{T}$ are called strongly equivalent if there exists a quasi-functor $\phi: \mathcal{B} \rightarrow \mathcal{B}^{\prime}$ such that the functors $\varepsilon^{\prime} \cdot H^{0}(\phi)$ and $\varepsilon$ are isomorphic.

It is important to know that an enhancement exists and is unique for a given triangulated category $\mathcal{T}$, because then its choice is not considered as an extra data. For example, in string theory, categories of D-branes arise as DG categories (actually $A_{\infty}$-categories), homotopy categories of which are equivalent to derived categories of coherent sheaves on some projective varieties. It is very useful to know that these equivalences can be lifted to the "DG level" as well; i.e., DG categories of D-branes are quasi-equivalent to natural enhancements of the derived categories of coherent sheaves.

We fix a field $k$ and all our categories are $k$-linear. Our main results are the following.

Theorem 1 (= Theorem 2.7). Let $\mathcal{A}$ be a small category which we consider as a $D G$ category and let $L \subset D(\mathcal{A})$ be a localizing subcategory with the quotient functor $\pi: D(\mathcal{A}) \rightarrow D(\mathcal{A}) / L$ that has a right adjoint (Bousfield localization) $\mu$. Assume that the following conditions hold:

a) for every $Y \in \mathcal{A}$ the object $\pi\left(h^{Y}\right) \in D(\mathcal{A}) / L$ is compact;

b) for every $Y, Z \in \mathcal{A}$ we have $\operatorname{Hom}\left(\pi\left(h^{Y}\right), \pi\left(h^{Z}\right)[i]\right)=0$ when $i<0$.

Then the triangulated category $D(\mathcal{A}) / L$ has a unique enhancement.

Theorem 2 (= Theorem 2.8). Let $\mathcal{A}$ be a small category which we consider as a $D G$ category and $L \subset D(\mathcal{A})$ be a localizing subcategory that is generated by compact objects $L^{c}=L \cap D(\mathcal{A})^{c}$. Assume that for the quotient functor $\pi: D(\mathcal{A}) \rightarrow D(\mathcal{A}) / L$ the following condition holds:

for every $Y, Z \in \mathcal{A}$ we have $\operatorname{Hom}\left(\pi\left(h^{Y}\right), \pi\left(h^{Z}\right)[i]\right)=0$ when $i<0$.

Then the triangulated subcategory of compact objects $(D(\mathcal{A}) / L)^{c}$ has a unique enhancement. 
Our main tool in the proof of Theorems 1 and 2 is the Drinfeld construction of a DG quotient of a DG category with its universal property [Dr].

For convenience, in Section 2 we collect all our results together. Sections 3-6 are devoted to proofs of two main Theorems 11 and 2 , First, we give some preliminary lemmas and present the main technical tool for the next sections, which is Proposition 3.4. Secondly, in Section 4 we construct a quasi-functor $\widetilde{\rho}$ (formula (4.2) ) which is a central object for all our considerations. After that in Sections 5 and 6 we prove the main theorems; we show how to apply Drinfeld Theorem 1.3 to the quasi-functor $\widetilde{\rho}$ and argue that the induced quasi-functor $\rho$ is actually a quasi-equivalence between different enhancements. In the end of Sections 5 and [6] we give more advanced and precise versions of Theorems 1 and 2 (see Theorems 5.4 and 6.4).

In Section 7 as a consequence of Theorem 10 we deduce the uniqueness of an enhancement for an unbounded derived category of an abelian Grothendieck category $\mathcal{C}$ under mild additional conditions on it (see Theorem 7.5). More precisely, we prove that the derived category $D(\mathcal{C})$ has a unique enhancement if the Grothendieck category $\mathcal{C}$ has a set of small generators which are compact objects in the derived category $D(\mathcal{C})$.

This result can be applied to the category of quasi-coherent sheaves on a quasicompact and quasi-separated scheme. We say that a quasi-compact and quasiseparated scheme $X$ has enough locally free sheaves if for any finitely presented sheaf $\mathcal{F}$ there is an epimorphism $\mathcal{E} \rightarrow \mathcal{F}$ with a locally free sheaf $\mathcal{E}$ of finite type. Theorem 7.5 immediately implies that the derived category of quasi-coherent sheaves $D($ Qcoh $X)$ has a unique enhancement if the quasi-compact and separated scheme $X$ has enough locally free sheaves (Theorem 7.6). In particular, this statement can be applied for any quasi-projective scheme (Corollary 17.8 ).

In Section 7 we show how to apply Theorem 2 to the subcategories of perfect complexes $\operatorname{Perf}(X)$. We proved that for any quasi-projective scheme $X$ over $k$ the triangulated category of perfect complexes $\operatorname{Perf}(X)$ has a unique enhancement (Theorem 7.9).

In Section 8 we introduce a notion of compactly approximated objects in a triangulated category and we prove that the triangulated category of compactly approximated objects $(D(\mathcal{A}) / L)^{c a}$ has a unique enhancement (Theorem 8.8). This result allows us to deduce the uniqueness of an enhancement for the bounded derived category of coherent sheaves $D^{b}(\operatorname{coh} X)$ on a quasi-projective scheme $X$ (Theorem 8.13).

In the case of projective varieties using results of [01, 02, we can prove stronger results. If $X$ is a projective scheme over $k$, then the bounded derived category $D^{b}(\operatorname{coh} X)$ and the triangulated category of perfect complexes $\operatorname{Perf}(X)$ have strongly unique enhancements (Theorem 9.9). This result is a consequence of a general statement about the bounded derived category of an exact category possessing an ample sequence of objects (Theorem 9.8).

As corollaries of these results we obtain a representation of fully faithful functors from categories of perfect complexes and bounded derived categories of coherent sheaves. Any complex of quasi-coherent sheaves $\mathcal{E}$. on the product $X \times Y$ determines a functor

$$
\Phi_{\mathcal{E}} \cdot(-)=\mathbf{R} p_{2 *}\left(\mathcal{E} \cdot \stackrel{\mathrm{L}}{\otimes} p_{1}^{*}(-)\right): D(\mathrm{Q} \operatorname{coh} X) \rightarrow D(\mathrm{Q} \operatorname{coh} Y)
$$


We show that our theorems on uniqueness of enhancements imply that if there is a fully faithful functor $K: \operatorname{Perf}(X) \rightarrow D(Q \operatorname{coh}(Y))$ for a quasi-projective scheme $X$ and a quasi-compact and separated scheme $Y$, then we can find an object $\mathcal{E} \in$ $D(\mathrm{Q} \operatorname{coh}(X \times Y))$ such that the restriction of the functor $\Phi_{\mathcal{E}}: D(\mathrm{Q} \operatorname{coh} X) \rightarrow$ $D($ Qcoh $Y)$ on $\operatorname{Perf}(X)$ is fully faithful too and $\Phi_{\mathcal{E}} \cdot\left(P^{\cdot}\right) \cong K\left(P^{\cdot}\right)$ for every $P^{\cdot} \in$ $\operatorname{Perf}(X)$. If, in addition, the functor $K$ sends $\operatorname{Perf}(X)$ to $\operatorname{Perf}(Y)$, then the functor $\Phi_{\mathcal{E}}$. is fully faithful, sends $\operatorname{Perf}(X)$ to $\operatorname{Perf}(Y)$, and $\mathcal{E}$ is isomorphic to an object of $D^{b}(\operatorname{coh}(X \times Y))$. Finally, if $X$ is a projective scheme such that the maximal torsion subsheaf $T_{0}\left(\mathcal{O}_{X}\right) \subset \mathcal{O}_{X}$ of dimension 0 is trivial, we show that the functor $\left.\Phi_{\mathcal{E}} \cdot\right|_{\operatorname{Perf}(X)}$ is isomorphic to $K$ (Corollary 9.13). For a projective scheme $X$ with $T_{0}\left(\mathcal{O}_{X}\right)=0$ and the bounded derived category of coherent sheaves $D^{b}(\operatorname{coh} X)$ we also proved that a fully faithful functor $K$ from $D^{b}(\operatorname{coh} X)$ to $D(\mathrm{Q} \operatorname{coh} Y)$ has the form $\Phi_{\mathcal{E}}$ if $K$ commutes with homotopy limits (see Corollaries 9.14 and 9.17 ).

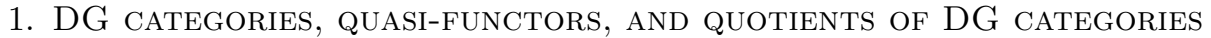

Our main reference for DG categories is $[\mathrm{K1}, \mathrm{Dr}$. Here we only recall a few points and introduce notation. Let $k$ be an arbitrary field. We will write $\otimes$ for the tensor product over $k$. All categories, DG categories, functors, DG functors, etc. are assumed to be $k$-linear.

A $D G$ category is a $k$-linear category $\mathcal{A}$ whose morphism spaces $\operatorname{Hom}(X, Y)$ are provided with a structure of a $\mathbb{Z}$-graded $k$-module and a differential $d: \operatorname{Hom}(X, Y) \rightarrow$ $\operatorname{Hom}(X, Y)$ of degree 1 , so that for every $X, Y, Z \in \mathrm{Ob} \mathcal{A}$ the composition $\operatorname{Hom}(Y, Z)$ $\otimes \operatorname{Hom}(X, Y) \rightarrow \operatorname{Hom}(X, Z)$ is the morphism of DG $k$-modules. The identity morphism $1_{X} \in \operatorname{Hom}(X, X)$ is closed of degree zero.

Using the supercommutativity isomorphism $S \otimes T \simeq T \otimes S$ in the category of DG $k$-modules one defines for every DG category $\mathcal{A}$ the opposite $D G$ category $\mathcal{A}^{\text {op }}$ with $\mathrm{Ob} \mathcal{A}^{o p}=\operatorname{Ob} \mathcal{A}$ and $\operatorname{Hom}_{\mathcal{A}^{o p}}(X, Y)=\operatorname{Hom}_{\mathcal{A}}(Y, X)$.

For a DG category $\mathcal{A}$ we denote by $H^{0}(\mathcal{A})$ and $H^{*}(\mathcal{A})$ its homotopy and graded homotopy categories, respectively. The homotopy category $H^{0}(\mathcal{A})$ has the same objects as the DG category $\mathcal{A}$ and its morphisms are defined by taking the 0 -th cohomology $H^{0}\left(\operatorname{Hom}_{\mathcal{A}}(X, Y)\right)$ of the complex $\operatorname{Hom}_{\mathcal{A}}(X, Y)$. The graded homotopy category $H^{*}(\mathcal{A})$ is defined by replacing each Hom complex in $\mathcal{A}$ by the direct sum of its cohomology groups.

As usual a $D G$ functor $\mathcal{F}: \mathcal{A} \rightarrow \mathcal{A}^{\prime}$ is given by a map $\mathcal{F}: \mathrm{Ob}(\mathcal{A}) \rightarrow \operatorname{Ob}\left(\mathcal{A}^{\prime}\right)$ and by morphisms of DG $k$-modules

$$
\mathcal{F}(X, Y): \operatorname{Hom}_{\mathcal{A}}(X, Y) \rightarrow \operatorname{Hom}_{\mathcal{A}}(\mathcal{F} X, \mathcal{F} Y), \quad X, Y \in \mathrm{Ob}(\mathcal{A})
$$

compatible with the composition and the units.

A DG functor $\mathcal{F}: \mathcal{A} \rightarrow \mathcal{B}$ is called a quasi-equivalence if $\mathcal{F}(X, Y)$ is a quasiisomorphism for all objects $X, Y$ of $\mathcal{A}$ and the induced functor $H^{0}(\mathcal{F}): H^{0}(\mathcal{A}) \rightarrow$ $H^{0}(\mathcal{B})$ is an equivalence. DG categories $\mathcal{A}$ and $\mathcal{B}$ are called quasi-equivalent if there exist DG categories $\mathcal{C}_{1}, \ldots, \mathcal{C}_{n}$ and a chain of quasi-equivalences $\mathcal{A} \leftarrow \mathcal{C}_{1} \rightarrow \cdots \leftarrow$ $\mathcal{C}_{n} \rightarrow \mathcal{B}$.

Given a small DG category $\mathcal{A}$ we define a right $D G \mathcal{A}$-module as a DG functor $M: \mathcal{A}^{o p} \rightarrow \mathcal{M o d}-k$, where $\mathcal{M}$ od $-k$ is the DG category of DG $k$-modules. We denote by $\mathcal{M}$ od $-\mathcal{A}$ the $\mathrm{DG}$ category of right DG $\mathcal{A}$-modules.

Denote by $\mathcal{A} c(\mathcal{A})$ the full DG subcategory of $\mathcal{M o d}-\mathcal{A}$ consisting of all acyclic DG modules. It is well known that the homotopy category of DG modules $H^{0}(\mathcal{M o d}-\mathcal{A})$ 
has a natural structure of a triangulated category and the homotopy category of acyclic complexes $H^{0}(\mathcal{A} c(\mathcal{A}))$ forms a full triangulated subcategory in it. The derived category $D(\mathcal{A})$ is the Verdier quotient of $H^{0}(\mathcal{M o d}-\mathcal{A})$ by the subcategory $H^{0}(\mathcal{A c}(\mathcal{A}))$.

For each object $Y$ of $\mathcal{A}$ we have the right module represented by $Y$,

$$
h^{Y}(-):=\operatorname{Hom}_{\mathcal{A}}(-, Y),
$$

which is called a representable DG module. This gives the Yoneda DG functor $h^{\bullet}: \mathcal{A} \rightarrow \mathcal{M o d}-\mathcal{A}$ that is full and faithful.

The DG $\mathcal{A}$-module is called free if it is isomorphic to a direct sum of DG modules of the form $h^{Y}[n]$, where $Y \in \mathcal{A}, n \in \mathbb{Z}$. A DG $\mathcal{A}$-module $P$ is called semi-free if it has a filtration $0=\Phi_{0} \subset \Phi_{1} \subset \ldots=P$ such that each quotient $\Phi_{i+1} / \Phi_{i}$ is free. The full DG subcategory of semi-free DG modules is denoted by $\mathcal{S F}(\mathcal{A})$. We denote by $\mathcal{S F}_{f g}(\mathcal{A}) \subset \mathcal{S F}(\mathcal{A})$ the full DG subcategory of finitely generated semifree DG modules i.e. $\Phi_{n}=P$ for some $n$ and $\Phi_{i+1} / \Phi_{i}$ is a finite direct sum of DG modules of the form $h^{Y}[n]$. We also denote by $\operatorname{Perf}(\mathcal{A})$ the DG category of perfect DG modules, i.e. the full DG subcategory of $\mathcal{S F}(\mathcal{A})$ consisting of all DG modules which are homotopy equivalent to a direct summand of a finitely generated semi-free DG module.

It is also natural to consider the category of h-projective DG modules. We call a DG $\mathcal{A}$-module $P$ h-projective (homotopically projective) if

$$
\operatorname{Hom}_{H^{0}(\mathcal{M o d}-\mathcal{A})}(P, N)=0
$$

for every acyclic DG module $N$ (by duality, we can define $h$-injective DG modules). Let $\mathcal{P}(\mathcal{A}) \subset \mathcal{M o d}-\mathcal{A}$ denote the full subcategory of h-projective objects. It can be easily checked that a semi-free DG-module is h-projective. For every DG $\mathcal{A}$ module $M$ there is a quasi-isomorphism $\mathbf{p} M \rightarrow M$ such that $\mathbf{p} M$ is a semi-free DG $\mathcal{A}$-module. Thus we obtain that the canonical DG functors $\mathcal{S F}(\mathcal{A}) \hookrightarrow \mathcal{P}(\mathcal{A}) \hookrightarrow$ $\mathcal{M o d}-\mathcal{A}$ induce equivalences $H^{0}(\mathcal{S F}(\mathcal{A})) \stackrel{\sim}{\rightarrow} H^{0}(\mathcal{P}(\mathcal{A})) \stackrel{\sim}{\rightarrow} D(\mathcal{A})$ of the triangulated categories (see $\mathrm{K} 1$ 3.1, $\mathrm{Hi}$ 2.2, $\mathrm{Dr}$ 13.2).

Let $\mathcal{F}: \mathcal{A} \rightarrow \mathcal{B}$ be a DG functor between DG categories. It induces the DG functors of restriction and extension of scalars

$$
\mathcal{F}_{*}: \operatorname{Mod}-\mathcal{B} \rightarrow \operatorname{Mod}-\mathcal{A}, \quad \mathcal{F}^{*}: \operatorname{Mod}-\mathcal{A} \rightarrow \mathcal{M o d}-\mathcal{B} .
$$

The DG functor $\mathcal{F}^{*}$ is an extension of $\mathcal{F}$ on the category of DG modules; i.e., the following diagram commutes:

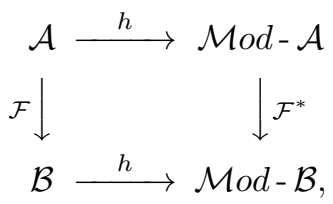

where the horizontal arrows are the Yoneda embeddings. The DG functors $\left(\mathcal{F}^{*}, \mathcal{F}_{*}\right)$ are adjoint: for $M \in \mathcal{M o d}-\mathcal{A}$ and $N \in \mathcal{M o d}-\mathcal{B}$ there are functorial isomorphisms

$$
\operatorname{Hom}_{\mathcal{M} \text { od }-\mathcal{B}}\left(\mathcal{F}^{*}(M), N\right) \cong \operatorname{Hom}_{\mathcal{M} o d-\mathcal{A}}\left(M, \mathcal{F}_{*}(N)\right) \text {. }
$$

The DG functor $\mathcal{F}^{*}$ preserves semi-free DG modules and $\mathcal{F}^{*}: \mathcal{S F}(\mathcal{A}) \rightarrow \mathcal{S F}(\mathcal{B})$ is a quasi-equivalence if $\mathcal{F}$ is such. The DG functor $\mathcal{F}_{*}$ preserves acyclic DG modules.

The DG functors $\mathcal{F}_{*}$ and $\mathcal{F}^{*}$ induce the corresponding derived functors

$$
F_{*}: D(\mathcal{B}) \rightarrow D(\mathcal{A}), \quad \mathbf{L} F^{*}: D(\mathcal{A}) \rightarrow D(\mathcal{B}) .
$$


There is a third DG functor $\mathcal{F}^{!}: \mathcal{M} o d-\mathcal{A} \rightarrow \operatorname{Mod}-\mathcal{B}$, which is a right adjoint to $\mathcal{F}_{*}$ and preserves h-injectives (see [ELO1]). It is defined by the following formula:

$$
\mathcal{F}^{!}(M)(X):=\operatorname{Hom}_{\mathcal{M} \text { od }-\mathcal{A}}\left(\mathcal{F}_{*}\left(h^{X}\right), M\right), \quad \text { where } X \in \mathcal{B} \text { and } M \in \mathcal{M o d}-\mathcal{A} \text {. }
$$

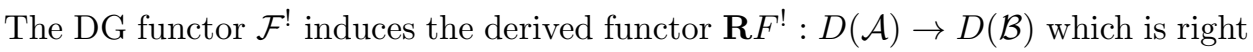
adjoint to $F_{*}$.

Let $\mathcal{A}$ and $\mathcal{B}$ be two small $\mathrm{DG}$ categories. Let $X$ be an $\mathcal{A}-\mathcal{B}$-bimodule, i.e. a DG $\mathcal{A}^{o p} \otimes \mathcal{B}$-module $X$. For each DG $\mathcal{A}$-module $M$, we obtain a DG $\mathcal{B}$-module $M \otimes_{\mathcal{A}} X$. The DG functor $(-) \otimes_{\mathcal{A}} X: \mathcal{M}$ od $-\mathcal{A} \rightarrow \mathcal{M}$ od $-\mathcal{B}$ admits a right adjoint $\operatorname{Hom}_{\mathcal{B}}(X,-)$. These functors do not respect quasi-isomorphisms in general, but they form a Quillen adjunction and the derived functors $(-) \stackrel{\mathbf{L}}{\otimes_{\mathcal{A}}} X$ and $\mathbf{R} \operatorname{Hom}_{\mathcal{B}}(X,-)$ form an adjoint pair of functors between derived categories $D(\mathcal{A})$ and $D(\mathcal{B})$.

Let DGcat ${ }_{k}$ be the category of small DG $k$-linear categories. It is known Ta] that it admits a structure of a cofibrantly generated model category whose weak equivalences are the quasi-equivalences. This shows in particular that the localization Hqe of DGcat ${ }_{k}$ with respect to the quasi-equivalences has small Hom-sets. This also gives that a morphism from $\mathcal{A}$ to $\mathcal{B}$ in the localization can be represented as $\mathcal{A} \leftarrow \mathcal{A}_{\text {cof }} \rightarrow \mathcal{B}$, where $\mathcal{A} \leftarrow \mathcal{A}_{\text {cof }}$ is a cofibrant replacement.

The morphism sets in the localization are much better described in terms of quasi-functors. Consider two DG categories $\mathcal{A}$ and $\mathcal{B}$. Denote by $\operatorname{rep}(\mathcal{A}, \mathcal{B})$ the full subcategory of the derived category $D\left(\mathcal{A}^{o p} \otimes \mathcal{B}\right)$ of $\mathcal{A}-\mathcal{B}$-bimodules formed by all bimodules $X$ such that the tensor functor

$$
(-) \stackrel{\mathrm{L}}{\otimes_{\mathcal{A}}} X: D(\mathcal{A}) \rightarrow D(\mathcal{B})
$$

takes every representable $\mathcal{A}$-module to an object which is isomorphic to a representable $\mathcal{B}$-module. We call such a bimodule a quasi-functor from $\mathcal{A}$ to $\mathcal{B}$. In other words, a quasi-functor is represented by a DG functor $\mathcal{A} \rightarrow \mathcal{M}$ od $-\mathcal{B}$ whose essential image consists of quasi-representable DG $\mathcal{B}$-modules ("quasi-representable" means quasi-isomorphic to a representable DG module). Since the category of quasirepresentable DG $\mathcal{B}$-modules is equivalent to $H^{0}(\mathcal{B})$ a quasi-functor $\mathcal{F} \in \operatorname{rep}(\mathcal{A}, \mathcal{B})$ defines a functor $H^{0}(\mathcal{F}): H^{0}(\mathcal{A}) \rightarrow H^{0}(\mathcal{B})$.

It is known (see [To ) that the morphisms from $\mathcal{A}$ to $\mathcal{B}$ in the localization of DGcat $_{k}$ with respect to the quasi-equivalences are in natural bijection with the isomorphism classes of $\operatorname{rep}(\mathcal{A}, \mathcal{B})$. Denote by DGcat a 2-category of DG categories with objects being small DG categories, 1-morphisms being quasi-functors, 2 -morphisms being morphisms of quasi-functors, i.e. morphisms in $D\left(\mathcal{A}^{o p} \otimes \mathcal{B}\right)$.

Example 1.1. Let $\mathcal{A}$ and $\mathcal{B}$ be two DG categories and $\Phi: \mathcal{A} \rightarrow \mathcal{M}$ od $-\mathcal{B}$ be a DG functor. Then $\Phi$ induces a quasi-functor from $\mathcal{A}$ to $\mathcal{S} \mathcal{F}(\mathcal{B})$. Indeed, every $Y \in \mathcal{A}$ defines a $\mathrm{DG} \mathcal{S} \mathcal{F}(\mathcal{B})$-module by the formula:

$$
P \mapsto \operatorname{Hom}_{\mathcal{M} \text { od }-\mathcal{B}}(P, \mathcal{F}(Y)), \quad \text { for any } \quad P \in \mathcal{S F}(\mathcal{A}) \text {. }
$$

If $Q \in \mathcal{S F}(\mathcal{B})$ is quasi-isomorphic to $\mathcal{F}(Y)$, then this DG $\mathcal{S F}(\mathcal{B})$-module is quasiisomorphic to $h^{Q} \in \mathcal{M o d}-\mathcal{S} \mathcal{F}(\mathcal{B})$. We will denote this quasi-functor by $\phi: \mathcal{A} \rightarrow$ $\mathcal{S F}(\mathcal{B})$.

For any DG category $\mathcal{A}$ there exist a DG category $\mathcal{A}^{\text {pre-tr }}$ that is called a pretriangulated hull and a canonical fully faithful DG functor $\mathcal{A} \hookrightarrow \mathcal{A}^{\text {pre-tr }}$. The idea of the definition of $\mathcal{A}^{\text {pre-tr }}$ is to formally add to $\mathcal{A}$ all shifts, all cones, cones of morphisms between cones, etc. The objects of this DG category are 'one-sided twisted 
complexes' (see [BK]). There is a canonical fully faithful DG functor (the Yoneda embedding) $\mathcal{A}^{\text {pre-tr }} \rightarrow \operatorname{Mod}-\mathcal{A}$, and under this embedding $\mathcal{A}^{\text {pre-tr }}$ is DG-equivalent to a DG category of finitely generated semi-free DG modules, which we denote by $\mathcal{S F}_{f g}(\mathcal{A})$.

Definition 1.2. We say that $\mathcal{A}$ is pretriangulated if for every object $X \in \mathcal{A}$ the object $X[n] \in \mathcal{A}^{\text {pre-tr }}$ is homotopy equivalent to an object of $\mathcal{A}$ and for every closed morphism $f$ in $\mathcal{A}$ of degree 0 the cone $\operatorname{Cone}(f) \in \mathcal{A}^{\text {pre-tr }}$ is homotopy equivalent to an object of $\mathcal{A}$. In other words $\mathcal{A}$ is pretriangulated if and only if the DG functor $\mathcal{A} \rightarrow \mathcal{A}^{\text {pre-tr }}$ is a quasi-equivalence.

Thus if $\mathcal{A}$ is pretriangulated the homotopy category $H^{0}(\mathcal{A})$ is triangulated. The DG category $\mathcal{A}^{\text {pre-tr }}$ is always pretriangulated, so $H^{0}\left(\mathcal{A}^{\text {pre-tr }}\right)$ is a triangulated category. We denote $\mathcal{A}^{\text {tr }}:=H^{0}\left(\mathcal{A}^{\text {pre-tr }}\right)$.

Notice that a quasi-functor $\mathcal{F} \in \operatorname{rep}(\mathcal{A}, \mathcal{B})$ defines a functor $\mathcal{A}^{\text {tr }} \rightarrow \mathcal{B}^{\text {tr }}$. Let us recall the main theorem in $[\mathrm{Dr}$.

Theorem $1.3(\overline{\mathrm{Dr}})$. Let $\mathcal{A}$ be a small $D G$ category and $\mathcal{B} \subset \mathcal{A}$ be a full $D G$ subcategory. For all pairs $(\mathcal{C}, \xi)$, where $\mathcal{C}$ is a $D G$ category and $\xi \in \operatorname{rep}(\mathcal{A}, \mathcal{C})$, the following properties are equivalent:

(i) the functor $H^{0}(\xi): H^{0}(\mathcal{A}) \rightarrow H^{0}(\mathcal{C})$ is essentially surjective, and the functor $\mathcal{A}^{\text {tr }} \rightarrow \mathcal{C}^{\text {tr }}$ corresponding to $\xi$ induces an equivalence $\mathcal{A}^{\text {tr }} / \mathcal{B}^{\text {tr }} \rightarrow \mathcal{C}^{\text {tr }}$;

(ii) for every $D G$ category $\mathcal{K}$ the functor $\operatorname{rep}(\mathcal{C}, \mathcal{K}) \rightarrow \operatorname{rep}(\mathcal{A}, \mathcal{K})$ corresponding to $\xi$ is fully faithful and $\Phi \in \operatorname{rep}(\mathcal{A}, \mathcal{K})$ belongs to its essential image if and only if the image of $\Phi$ in $\operatorname{rep}(\mathcal{B}, \mathcal{K})$ is zero.

A pair $(\mathcal{C}, \xi)$ satisfying (i),(ii) exists and is unique in the sense of DGcat: given another such pair $\left(\mathcal{C}^{\prime}, \xi^{\prime}\right)$ there exists a quasi-functor $\delta \in \operatorname{rep}\left(\mathcal{C}, \mathcal{C}^{\prime}\right)$ inducing an equivalence $H^{0}(\delta): H^{0}(\mathcal{C}) \stackrel{\sim}{\longrightarrow} H^{0}\left(\mathcal{C}^{\prime}\right)$ and such that the quasi-functors $\xi^{\prime}$ and $\delta \cdot \xi$ are isomorphic.

Remark 1.4. Although the above theorem is stated in the language of quasi-functors, it is important for us that one can choose a pair $(\mathcal{C}, \xi)$, where $\xi: \mathcal{A} \rightarrow \mathcal{C}$ is a DG functor and not just a quasi-functor. Indeed, recall Drinfeld's construction of the DG quotient $\mathcal{A} / \mathcal{B}$ : it is obtained from $\mathcal{A}$ by adding for every object $U \in \mathcal{B}$ a morphism $\epsilon_{U}: U \rightarrow U$ of degree -1 such that $d\left(\epsilon_{U}\right)=\operatorname{id}_{U}$ (no new objects and no new relations between morphisms are added). Thus in particular $\mathcal{A}$ is a DG subcategory of $\mathcal{A} / \mathcal{B}$ and $\xi: \mathcal{A} \rightarrow \mathcal{A} / \mathcal{B}$ is the inclusion $\mathrm{DG}$ functor.

Lemma 1.5. Let $\mathcal{A}$ be a small $D G$ category and let $\mathcal{B} \subset \mathcal{A}$ be a full $D G$ subcategory. Assume that $\mathcal{A}$ is pretriangulated. Then the Drinfeld $D G$ quotient $\mathcal{A} / \mathcal{B}$ is also pretriangulated.

Proof. The canonical embedding $H^{0}(\mathcal{A} / \mathcal{B}) \rightarrow(\mathcal{A} / \mathcal{B})^{\text {tr }}$ is full and faithful. We need to prove that it is essentially surjective. The quotient DG functor $\xi: \mathcal{A} \rightarrow \mathcal{A} / \mathcal{B}$ induces the DG functor $\xi^{\text {pre-tr }}: \mathcal{A}^{\text {pre-tr }} \rightarrow(\mathcal{A} / \mathcal{B})^{\text {pre-tr }}$ so that the natural diagram

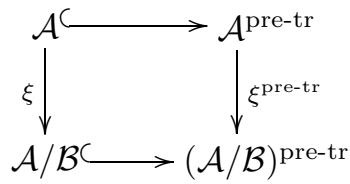


commutes. It induces the commutative diagram

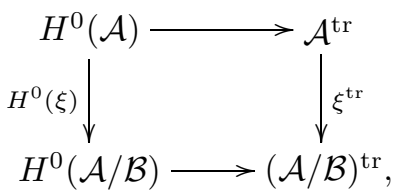

where the upper horizontal arrow is an equivalence by our assumption and the right vertical arrow identifies $(\mathcal{A} / \mathcal{B})^{\text {tr }}$ with the Verdier quotient of $\mathcal{A}^{\text {tr }}$ by $\mathcal{B}^{\text {tr }}$ according to Theorem 1.3. Hence the composition of these two functors is essentially surjective, which proves the lemma.

Definition 1.6. Let $\mathcal{T}$ be a category. An object $Y \in \mathcal{T}$ is called compact (in $\mathcal{T}$ ) if $\operatorname{Hom}_{\mathcal{T}}(Y,-)$ commutes with arbitrary (existing in $\mathcal{T}$ ) direct sums; i.e., for each family of objects $\left\{X_{i}\right\} \subset \mathcal{T}$ such that $\bigoplus_{i} X_{i}$ exists the canonical map

$$
\bigoplus_{i} \operatorname{Hom}\left(Y, X_{i}\right) \longrightarrow \operatorname{Hom}\left(Y, \bigoplus_{i} X_{i}\right)
$$

is an isomorphism.

Denote by $\mathcal{T}^{c} \subset \mathcal{T}$ the full subcategory consisting of all compact objects in $\mathcal{T}$.

Definition 1.7. Let $\mathcal{T}$ be a triangulated category that admits arbitrary direct sums. A set $S \subset \mathcal{T}^{c}$ is called a set of compact generators if any object $X \in \mathcal{T}$ such that $\operatorname{Hom}(Y, X[n])=0$ for all $Y \in S$ and all $n \in \mathbb{Z}$ is a zero object.

Remark 1.8. Since $\mathcal{T}$ admits arbitrary direct sums it can be proved that the property that $S \subset \mathcal{T}^{c}$ is a set of compact generators is equivalent to the following property: the category $\mathcal{T}$ coincides with the smallest full triangulated subcategory containing $S$ and closed under direct sums.

Example 1.9. Let $\mathcal{A}$ be a small DG category. The set $\left\{h^{Y}\right\}_{Y \in \mathcal{A}}$ is a set of compact generators of $D(\mathcal{A})$ and the subcategory of compact objects $D(\mathcal{A})^{c}$ coincides with the subcategory of perfect DG modules $\operatorname{Perf}(\mathcal{A})$.

There is another notion of a set of generators. It is called a set of classical generators.

Definition 1.10. Let $\mathcal{T}$ be a triangulated category. We say that a set $S \subset \mathcal{T}$ is a set of classical generators for $\mathcal{T}$ if the category $\mathcal{T}$ coincides with the smallest triangulated subcategory of $\mathcal{T}$ which contains $S$ and is closed under direct summands.

Remark 1.11. These two definitions of a set of generators are closely related to each other. Assume that a triangulated category $\mathcal{T}$, which admits arbitrary direct sums, is compactly generated by the set of compact objects $\mathcal{T}^{c}$. In this situation a set $S \subset \mathcal{T}^{c}$ is a set of compact generators of $\mathcal{T}$ if and only if this set $S$ is a set of classical generators of the subcategory of compact objects $\mathcal{T}^{c}$. This is proved in [N1. Thus the set $\left\{h^{Y}\right\}_{Y \in \mathcal{A}}$ from Example 1.9 is a set of classical generators of the subcategory of compact objects $D(\mathcal{A})^{c} \cong \operatorname{Perf}(\mathcal{A})$.

We will recall the definition of homotopy colimits in triangulated categories. Namely, let $\mathcal{T}$ be a triangulated category and

$$
X_{0} \stackrel{i_{0}}{\longrightarrow} X_{1} \stackrel{i_{1}}{\longrightarrow} \cdots \longrightarrow X_{n} \stackrel{i_{n}}{\longrightarrow} X_{n+1} \longrightarrow \cdots
$$

be a sequence of morphisms in $\mathcal{T}$. 
Definition 1.12. Assume that the direct sum $\bigoplus_{n} X_{n}$ exists in $\mathcal{T}$. The homotopy limit of this sequence hocolim $X_{n} \in \mathcal{T}$ is by definition given, up to noncanonical isomorphism, by a triangle

$$
\bigoplus_{n} X_{n} \stackrel{\left(1,-i_{n}\right)}{\longrightarrow} \bigoplus_{n} X_{n} \longrightarrow \operatorname{hocolim}_{\longrightarrow} X_{n} \longrightarrow\left(\bigoplus_{n} X_{n}\right)[1] .
$$

If $\mathcal{A}$ is a DG category and maps $i_{n}: X_{n} \rightarrow X_{n+1}$ are closed morphisms of degree zero in $\operatorname{Mod}-\mathcal{A}$, then one has the usual $\underset{\operatorname{colim}}{\longrightarrow} X_{n}$ in the category $Z^{0}(\mathcal{M} o d-\mathcal{A})$ which is isomorphic to hocolim $X_{n}$ in $D(\mathcal{A})$. The category $Z^{0}(\mathcal{M o d}-\mathcal{A})$ has the same object as $\operatorname{Mod}-\mathcal{A}$ and morphisms are closed morphisms of degree 0 (it is an abelian category).

Definition 1.13. Let $\mathcal{T}$ be a triangulated category with arbitrary direct sums. A strictly full triangulated subcategory $\mathcal{S} \subset \mathcal{T}$ is called localizing if it is closed under arbitrary direct sums.

Remark 1.14. If a subcategory $\mathcal{S}$ is localizing it is known that the quotient triangulated category $\mathcal{T} / \mathcal{S}$ has arbitrary direct sums and the quotient functor $\mathcal{T} \rightarrow \mathcal{T} / \mathcal{S}$ preserves direct sums (see N3, Cor. 3.2.11). For example, the triangulated category $H^{0}(\mathcal{M o d}-\mathcal{A})$ has arbitrary direct sums and $H^{0}(\mathcal{A} c(\mathcal{A}))$ is a localizing subcategory in $H^{0}(\mathcal{M o d}-\mathcal{A})$. Hence, the derived category $D(\mathcal{A})$ also has arbitrary direct sums.

The following propositions (and their proofs) are essentially equal to Lemma 4.2 in [K1].

Proposition 1.15. Let $\mathcal{F}: \mathcal{B} \hookrightarrow \mathcal{C}$ be a full embedding of $D G$ categories. and let $\mathcal{F}^{*}: \mathcal{S F}(\mathcal{B}) \rightarrow \mathcal{S F}(\mathcal{C})$ be the extension $D G$ functor. Then the induced derived functor $\mathbf{L} F^{*}=H^{0}\left(\mathcal{F}^{*}\right): D(\mathcal{B}) \rightarrow D(\mathcal{C})$ is fully faithful. If, in addition, the category $H^{0}(\mathcal{C})$ is classically generated by $\mathrm{Ob} \mathcal{B}$, then $\mathbf{L} F^{*}$ is an equivalence.

Proof. The functor $\mathbf{L} F^{*}$ commutes with direct sums and on $\mathcal{B} \subset \mathcal{D}(\mathcal{B})$ coincides with the functor $H^{0}(\mathcal{F})$ that is fully faithful.

Let $Y \in \mathcal{B}$. The objects $X \in D(\mathcal{B})$ for which the map

$$
\operatorname{Hom}_{D(\mathcal{B})}(Y, X[n]) \longrightarrow \operatorname{Hom}_{D(\mathcal{C})}\left(\mathbf{L} F^{*}(Y), \mathbf{L} F^{*}(X)[n]\right)
$$

is bijective for all $n$ form a triangulated subcategory of $D(\mathcal{B})$ which contains the generating set $\mathrm{ObB}$ and is closed under direct sums (since $Y$ and $\mathbf{L} F^{*}(Y)$ are compact and $\mathbf{L} F^{*}$ commutes with direct sums). So this subcategory coincides with the whole $D(\mathcal{B})$. The same argument shows that for a fixed $X \in D(\mathcal{B})$ the map

$$
\operatorname{Hom}_{D(\mathcal{B})}\left(X^{\prime}, X\right) \longrightarrow \operatorname{Hom}_{D(\mathcal{C})}\left(\mathbf{L} F^{*}\left(X^{\prime}\right), \mathbf{L} F^{*}(X)\right)
$$

is bijective for all $X^{\prime} \in D(\mathcal{B})$. Hence $\mathbf{L} F^{*}$ is fully faithful.

If now the category $H^{0}(\mathcal{C})$ is classically generated by $\mathrm{Ob} \mathcal{B}$, then it is contained in the essential image of $\mathbf{L} F^{*}$. On the other hand, the set $\mathrm{Ob} \mathcal{C}$ compactly generates $D(\mathcal{C})$. Hence the whole category $D(\mathcal{C})$ is in the essential image of $\mathbf{L} F^{*}$, because $D(\mathcal{B})$ contains arbitrary direct sums and $\mathbf{L} F^{*}$ commutes with direct sums.

Let $\mathcal{C}$ be a pretriangulated DG category and $\mathcal{B} \subset \mathcal{C}$ be a full DG subcategory. Consider the canonical DG functor

$$
\Phi: \mathcal{C} \rightarrow \mathcal{M o d}-\mathcal{B}, \quad \text { where } \Phi(X)(B)=\operatorname{Hom}_{\mathcal{C}}(B, X) \text { for } B \in \mathcal{B}, X \in \mathcal{C} .
$$


If we denote by $\mathcal{J}$ the full embedding of $\mathcal{B}$ to $\mathcal{C}$, then the DG functor $\Phi$ is the composition of the Yoneda DG functor $h^{\bullet}: \mathcal{C} \rightarrow \mathcal{M o d}-\mathcal{C}$ and the restriction functor $\mathcal{J}_{*}: \mathcal{M o d}-\mathcal{C} \rightarrow \mathcal{M o d}-\mathcal{B}$.

The DG functor $\Phi$ induces a quasi-functor $\phi: \mathcal{C} \rightarrow \mathcal{S F}(\mathcal{B})$ (see Remark 1.1) and the functor $H^{0}(\Phi): H^{0}(\mathcal{C}) \rightarrow H^{0}(\mathcal{M o d}-\mathcal{B})$. The homotopy functor $H^{0}(\phi):$ $H^{0}(\mathcal{C}) \rightarrow D(\mathcal{B})$ is the composition of $H^{0}(\Phi)$ with the localization functor $Q$ : $H^{0}(\mathcal{M o d}-\mathcal{B}) \rightarrow D(\mathcal{B})$.

Proposition 1.16. Let $\mathcal{B} \subset \mathcal{C}$ and $\phi: \mathcal{C} \rightarrow \mathcal{S F}(\mathcal{B})$ be as above. Assume that the triangulated category $H^{0}(\mathcal{C})$ is idempotent complete and is classically generated by the set $\mathrm{Ob} \mathcal{B}$. Then the functor $H^{0}(\phi)$ induces an equivalence between $H^{0}(\mathcal{C})$ and the subcategory of compact objects $D(\mathcal{B})^{c} \cong \operatorname{Perf}(\mathcal{B})$.

Proof. Indeed, if $Y, Z \in \mathcal{B} \subset \mathcal{C}$, then

$$
\operatorname{Hom}_{H^{0}(\mathcal{M o d}-\mathcal{B})}\left(H^{0}(\Phi)(Y), H^{0}(\Phi)(Z)[n]\right)=\operatorname{Hom}_{H^{0}(\mathcal{C})}(Y, Z[n])
$$

by Yoneda. Now, since $\Phi(Y)=h^{Y} \in H^{0}(\mathcal{M o d}-\mathcal{B})$ is h-projective we have

$$
\operatorname{Hom}_{H^{0}(\mathcal{M} o d-\mathcal{B})}\left(H^{0}(\Phi)(Y), H^{0}(\Phi)(Z)[n]\right) \cong \operatorname{Hom}_{D(\mathcal{B})}\left(H^{0}(\phi)(Y), H^{0}(\phi)(Z)[n]\right) .
$$

Since the category $H^{0}(\mathcal{C})$ is classically generated by $\mathrm{Ob} \mathcal{B}$ we get that the functor $H^{0}(\phi)$ is fully faithful. Since $H^{0}(\mathcal{C})$ is idempotent complete and $D(\mathcal{B})^{c}$ is classically generated by $\operatorname{Ob} \mathcal{B}$ we obtain that the subcategory $D(\mathcal{B})^{c}$ is the essential image of the functor $H^{0}(\phi)$.

Proposition 1.17. Let $\mathcal{B} \subset \mathcal{C}$, and $\phi: \mathcal{C} \rightarrow \mathcal{S F}(\mathcal{B})$ be as above. Assume that $H^{0}(\mathcal{C})$ contains arbitrary direct sums and $\mathrm{Ob} \mathcal{B}$ forms a set of compact generators of $H^{0}(\mathcal{C})$. Then the functor $H^{0}(\phi): H^{0}(\mathcal{C}) \stackrel{\sim}{\rightarrow} D(\mathcal{B})$ is an equivalence of triangulated categories.

Proof. First notice that the functor $H^{0}(\phi)$ commutes with direct sums. Indeed, since $Q$ commutes with direct sums (see Remark 1.14) it suffices to prove that $H^{0}(\Phi)$ does. Fix $Y \in \mathcal{B}$ and a set $\left\{X_{i}\right\} \subset \mathcal{C}$. Since $Y$ is compact in $H^{0}(\mathcal{C})$ we have the following isomorphisms, which are functorial in $Y$ :

$$
\begin{aligned}
H^{0}(\Phi)\left(\bigoplus X_{i}\right)(Y) & =H^{0} \operatorname{Hom}_{\mathcal{C}}\left(Y, \bigoplus X_{i}\right) \\
& =\bigoplus H^{0} \operatorname{Hom}_{\mathcal{C}}\left(Y, X_{i}\right)=\bigoplus H^{0}(\Phi)\left(X_{i}\right)(Y) .
\end{aligned}
$$

Hence $H^{0}(\Phi)\left(\bigoplus X_{i}\right)=\bigoplus H^{0}(\Phi)\left(X_{i}\right)$.

Note that the exact functor $H^{0}(\phi)$ maps the set $\mathrm{Ob} \mathcal{B}$ of compact generators of $H^{0}(\mathcal{C})$ to the set $\left\{h^{Y}\right\}_{Y \in \mathcal{B}}$ of compact generators of $D(\mathcal{B})$. By Remark 1.11 and Proposition 1.16 it induces an equivalence between subcategories of compact objects $H^{0}(\mathcal{C})^{c}$ and $D(\mathcal{B})^{c}$.

The same argument as in the proof of Proposition 1.15 gives us that the functor $H^{0}(\phi)$ is fully faithful. Since, in addition, $H^{0}(\mathcal{C})$ contains arbitrary direct sums and $H^{0}(\phi)$ commutes with direct sums it follows that $H^{0}(\phi)$ is essentially surjective.

Let $\mathcal{T}$ be a triangulated category with small Hom-sets; i.e., Hom between any two objects should be a set. Assume that $\mathcal{T}$ admits arbitrary direct sums and let $\mathcal{S} \subset \mathcal{T}$ be a localizing triangulated subcategory. We can consider the Verdier quotient $\mathcal{T} / \mathcal{S}$ with a natural localization map $\pi: \mathcal{T} \rightarrow \mathcal{T} / \mathcal{S}$. Notice however that Hom-sets in $\mathcal{T} / \mathcal{S}$ need not be small. It is known (Remark 1.14) that the category 
$\mathcal{T} / \mathcal{S}$ also has arbitrary direct sums and, moreover, the functor $\pi$ preserves direct sums.

Assume that the Verdier quotient $\mathcal{T} / \mathcal{S}$ is a category with small Hom-sets. If the triangulated category $\mathcal{T}$ has a set of compact generators, then the Brown representability theorem holds for $\mathcal{T}$ and the quotient functor $\pi: \mathcal{T} \rightarrow \mathcal{T} / \mathcal{S}$ has a right adjoint $\mu: \mathcal{T} / \mathcal{S} \rightarrow \mathcal{T}$ (see [N3], Ex.8.4.5). This adjoint is called the Bousfield localization functor.

Definition 1.18. Let $\mathcal{T}$ be a triangulated category with small Hom-sets. Let $\mathcal{S}$ be a thick subcategory. We say that a Bousfield localization functor exists for the pair $\mathcal{S} \subset \mathcal{T}$ when there is a right adjoint to the natural functor $\pi: \mathcal{T} \rightarrow \mathcal{T} / \mathcal{S}$. We will call the adjoint the Bousfield localization functor, and denote it $\mu: \mathcal{T} / \mathcal{S} \rightarrow \mathcal{T}$.

Let us summarize the facts about Bousfield localization we will need in the following proposition.

Proposition 1.19. Let $\mathcal{T}$ be a compactly generated triangulated category with small Hom-sets that admits arbitrary direct sums. Let $\mathcal{S} \subset \mathcal{T}$ be a localizing triangulated subcategory and $\pi: \mathcal{T} \rightarrow \mathcal{T} / \mathcal{S}$ be the quotient functor. Assume that the quotient $\mathcal{T} / \mathcal{S}$ is a category with small Hom-sets. Then

a) there is a right adjoint functor $\mu: \mathcal{T} / \mathcal{S} \rightarrow \mathcal{T}$;

b) the functor $\mu$ is full and faithful;

c) if for every compact object $Y \in \mathcal{T}$, the object $\pi(Y)$ is compact in $\mathcal{T} / \mathcal{S}$, then the functor $\mu$ preserves direct sums;

d) if for every compact object $Y \in \mathcal{T}$, the object $\pi(Y)$ is compact in $\mathcal{T} / \mathcal{S}$ and $\mathcal{T}$ is compactly generated by a set $R \subset \mathcal{T}^{c}$, then $\mathcal{T} / \mathcal{S}$ is also compactly generated by $\pi(R)$.

Proof. a) This is a consequence of the Brown representability theorem (N], Th. 8.4.4).

b) This is also a general statement which says that if the right adjoint functor to a localization exists, then it is fully faithful ([N3], Lemma 9.1.7).

c) If the object $\pi(Y)$ is compact, then

$$
\begin{aligned}
\operatorname{Hom}\left(Y, \mu\left(\bigoplus X_{i}\right)\right) & \cong \operatorname{Hom}\left(\pi(Y), \bigoplus X_{i}\right) \cong \bigoplus \operatorname{Hom}\left(\pi(Y), X_{i}\right) \\
& \cong \bigoplus \operatorname{Hom}\left(Y, \mu\left(X_{i}\right)\right) \cong \operatorname{Hom}\left(Y, \bigoplus \mu\left(X_{i}\right)\right)
\end{aligned}
$$

for every $Y \in \mathcal{T}^{c}$. Since $\mathcal{T}$ is compactly generated we obtain that $\bigoplus \mu\left(X_{i}\right) \rightarrow$ $\mu\left(\bigoplus X_{i}\right)$ is an isomorphism.

d) If $\operatorname{Hom}(\pi(Y), X[n])=0$ for all $Y \in R$ and all $n \in \mathbb{Z}$, then $\operatorname{Hom}(Y, \mu(X)[n])=$ 0 . Since $R \subset \mathcal{T}^{c}$ is a set of compact generators, then $\mu(X)=0$. Hence $X=$ 0 , because $\mu$ is fully faithful. Therefore $\pi(R)$ is a set of compact generators for $\mathcal{T} / \mathcal{S}$.

Remark 1.20. Let $\mathcal{A}$ be a DG category. The objects $\left\{h^{Y}\right\}_{Y \in \mathcal{A}}$ form a set of compact generators of $D(\mathcal{A})$. Let $L \subset D(\mathcal{A})$ be a localizing subcategory, and $\pi: D(\mathcal{A}) \rightarrow$ $D(\mathcal{A}) / L$ be the quotient functor. Assume that the Verdier quotient $D(\mathcal{A}) / L$ is a category with small Hom-sets. Then we can apply Proposition 1.19 and get a Bousfield localization functor $\mu: D(\mathcal{A}) / L \rightarrow D(\mathcal{A})$, which is fully faithful. If for every $Y \in \mathcal{A}$ the object $\pi\left(h^{Y}\right)$ is compact in $D(\mathcal{A}) / L$, then the objects $\left\{\pi\left(h^{Y}\right)\right\}_{Y \in \mathcal{A}}$ compactly generate the category $D(\mathcal{A}) / L$ and the functor $\mu$ preserves direct sums. 
If a localizing subcategory $\mathcal{S} \subset \mathcal{T}$ is compactly generated by the set of objects $\mathcal{S} \cap \mathcal{T}^{c}$, then the quotient category $\mathcal{T} / \mathcal{S}$ has small Hom-sets ([N3], Cor.4.4.3) and we can say even more ([N1], Th.2.1, and [N3], Th.4.4.9).

Theorem 1.21 (则). Let $\mathcal{T}$ be a compactly generated triangulated category admitting arbitrary direct sums and let $\mathcal{S}$ be a localizing triangulated subcategory which is generated by a subset $S \subset \mathcal{T}^{c}$ of compact objects. Then

(1) $\mathcal{T} / \mathcal{S}$ has small Hom-sets and is compactly generated;

(2) $\mathcal{T}^{c}$ maps to $(\mathcal{T} / \mathcal{S})^{c}$ under the quotient functor;

(3) the induced functor $\mathcal{T}^{c} / \mathcal{S}^{c} \rightarrow(\mathcal{T} / \mathcal{S})^{c}$ is fully faithful;

(4) $(\mathcal{T} / \mathcal{S})^{c}$ is the idempotent completion of $\mathcal{T}^{c} / \mathcal{S}^{c}$.

Remark 1.22. One of our main tools will be Theorem 1.3 above, which assumes that we work with small categories. More precisely, we will need to apply the DG localization to the DG category $\operatorname{Mod}-\mathcal{A}$, where $\mathcal{A}$ is a small category. Thus we will choose universes $\mathbb{U} \in \mathbb{V} \in \cdots$, assume that the category $\mathcal{A}$ is a $\mathbb{U}$-small $\mathbb{U}$ category and will consider the DG category $\mathcal{M o d}_{\mathbb{U}}-\mathcal{A}$ of $\mathbb{U}$-small DG $\mathcal{A}$-modules. Thus $\mathcal{M o d}_{\mathbb{U}}-\mathcal{A}$ is a DG $\mathbb{U}$-category, and we explain in the Appendix that it is DG equivalent to a $\mathbb{V}$-small DG category $\mathcal{M} o d_{\mathbb{U}}^{\text {str }}-\mathcal{A}$ of strict $\mathbb{U}$-small DG $\mathcal{A}$-modules. So we may apply the DG localization to $\mathcal{M} o d_{\mathbb{U}}^{\text {str }}-\mathcal{A}$ instead of $\mathcal{M} o d_{\mathbb{U}}-\mathcal{A}$.

However we decided not to mention explicitly the $\mathbb{U}$-smallness issue in the main body of the text. Therefore we add an appendix which contains all the relevant statements and in particular it has the $\mathbb{U}$-small version of the Brown representability theorem, etc.

\section{ENHANCEMENTS OF TRIANGULATED CATEGORIES AND FORMULATION OF MAIN RESULTS}

Definition 2.1. Let $\mathcal{T}$ be a triangulated category. An enhancement of $\mathcal{T}$ is a pair $(\mathcal{B}, \varepsilon)$, where $\mathcal{B}$ is a pretriangulated DG category and $\varepsilon: H^{0}(\mathcal{B}) \stackrel{\sim}{\rightarrow} \mathcal{T}$ is an equivalence of triangulated categories.

Definition 2.2. The category $\mathcal{T}$ has a unique enhancement if it has one and for any two enhancements $(\mathcal{B}, \varepsilon)$ and $\left(\mathcal{B}^{\prime}, \varepsilon^{\prime}\right)$ of $\mathcal{T}$ there exists a quasi-functor $\phi$ : $\mathcal{B} \rightarrow \mathcal{B}^{\prime}$ which induces an equivalence $H^{0}(\phi): H^{0}(\mathcal{B}) \stackrel{\sim}{\longrightarrow} H^{0}\left(\mathcal{B}^{\prime}\right)$. In this case the enhancements $(\mathcal{B}, \varepsilon)$ and $\left(\mathcal{B}^{\prime}, \varepsilon^{\prime}\right)$ are called equivalent.

Definition 2.3. Enhancements $(\mathcal{B}, \varepsilon)$ and $\left(\mathcal{B}^{\prime}, \varepsilon^{\prime}\right)$ of $\mathcal{T}$ are called strongly equivalent if there exists a quasi-functor $\phi: \mathcal{B} \rightarrow \mathcal{B}^{\prime}$ such that the functors $\varepsilon^{\prime} \cdot H^{0}(\phi)$ and $\varepsilon$ are isomorphic.

Here are some examples of existence of canonical enhancements.

Example 2.4. Let $\mathfrak{A}$ be an abelian category. Denote by $\mathcal{C}_{d g}(\mathfrak{A})$ the DG category of complexes over $\mathfrak{A}$, and by $D(\mathfrak{A})$ the derived category of $\mathfrak{A}$. Let $L \subset D(\mathfrak{A})$ be a localizing subcategory. Consider the full pretriangulated DG subcategory $\mathcal{L} \subset \mathcal{C}_{d g}(\mathfrak{A})$ such that $\mathrm{Ob} \mathcal{L}=\mathrm{Ob} L$. Let $\mathcal{C}_{d g}(\mathfrak{A}) / \mathcal{L}$ be the corresponding DG quotient. Then by Theorem 1.3 above there is a natural equivalence $F: H^{0}\left(\mathcal{C}_{d g}(\mathfrak{A}) / \mathcal{L}\right) \stackrel{\sim}{\longrightarrow} D(\mathfrak{A}) / L$ of triangulated categories. Hence $\left(\mathcal{C}_{d g}(\mathfrak{A}) / \mathcal{L}, F\right)$ is a canonical enhancement of $D(\mathfrak{A}) / L$.

We also can consider a slight variation. 
Example 2.5. Let $\mathcal{A}$ be a DG category. If $L \subset D(\mathcal{A})$ is a localizing subcategory, the quotient category $D(\mathcal{A}) / L$ has a canonical enhancement. Namely, let $\mathcal{L} \subset \operatorname{Mod}-\mathcal{A}$ be the full DG subcategory which has the same objects as $L$. Let $\operatorname{Mod}-\mathcal{A} / \mathcal{L}$ be the DG quotient category. Then there is a natural equivalence $F: H^{0}(\mathcal{M o d}-\mathcal{A} / \mathcal{L}) \stackrel{\sim}{\longrightarrow} D(\mathcal{A}) / L$ of triangulated categories; i.e., $(\mathcal{M}$ od $-\mathcal{A} / \mathcal{L}, F)$ is an enhancement of $D(\mathcal{A}) / L$. Consider the DG subcategories $\mathcal{S} \mathcal{F}(\mathcal{A}) \subset \mathcal{P}(\mathcal{A}) \subset$ $\mathcal{M o d}-\mathcal{A}$ of semi-free and h-projective DG modules, respectively. Then the natural DG functors

$$
\mathcal{S F}(\mathcal{A}) / \mathcal{L} \cap \mathcal{S F}(\mathcal{A}) \longrightarrow \mathcal{P}(\mathcal{A}) / \mathcal{L} \cap \mathcal{P}(\mathcal{A}) \longrightarrow \mathcal{M o d}-\mathcal{A} / \mathcal{L}
$$

are quasi-equivalences, so that $\mathcal{S} \mathcal{F}(\mathcal{A}) / \mathcal{L} \cap \mathcal{S F}(\mathcal{A})$ and $\mathcal{P}(\mathcal{A}) / \mathcal{L} \cap \mathcal{P}(\mathcal{A})$ are other enhancements of $D(\mathcal{A}) / L$ that are equivalent to $\operatorname{Mod}-\mathcal{A} / \mathcal{L}$.

For a triangulated category it is natural to ask if it has an enhancement. Next is the question of uniqueness.

First, let us prove the following not difficult and very natural proposition.

Proposition 2.6. Let $\mathcal{A}$ be a small category which we consider as a $D G$ category and $D(\mathcal{A})$ and $\operatorname{Perf}(A)=D(\mathcal{A})^{c}$ be the derived category of $D G \mathcal{A}$-modules and its subcategory of perfect $D G$ modules, respectively. Then each of them has a unique enhancement.

Proof. The canonical DG functors $S F(\mathcal{A}) \hookrightarrow \mathcal{P}(\mathcal{A}) \hookrightarrow \mathcal{M o d}-\mathcal{A} / \mathcal{A} c(\mathcal{A})$ are quasiisomorphisms and gives equivalent enhancements of the derived category $D(\mathcal{A})$.

Let $\mathcal{D}$ be a pretriangulated DG category and $\epsilon: D(\mathcal{A}) \stackrel{\sim}{\rightarrow} H^{0}(\mathcal{D})$ be an equivalence of triangulated categories. Denote by $\mathcal{B} \subset \mathcal{D}$ the full DG subcategory with the set of objects $\left\{\epsilon\left(h^{Y}\right)\right\}_{Y \in \mathcal{A}}$.

Denote by $\tau_{\leq 0} \mathcal{B}$ the DG subcategory with the same objects as $\mathcal{B}$ and morphisms

$$
\operatorname{Hom}_{\tau \leq 0} \mathcal{B}(M, N)=\tau_{\leq 0} \operatorname{Hom}_{\mathcal{B}}(M, N),
$$

where $\tau_{\leq 0}$ is the usual truncation of complexes:

$$
\tau_{\leq 0}\left\{\cdots \longrightarrow C^{-1} \longrightarrow C^{0} \stackrel{d^{0}}{\longrightarrow} C^{1} \longrightarrow \cdots\right\}=\left\{\cdots \longrightarrow C^{-1} \longrightarrow \operatorname{Ker}\left(d^{0}\right) \longrightarrow 0\right\} .
$$

We have the obvious diagram of DG categories and DG functors

$$
\mathcal{A}=H^{0}(\mathcal{B}) \stackrel{p}{\longleftarrow} \tau_{\leq 0} \mathcal{B} \stackrel{i}{\longrightarrow} \mathcal{B},
$$

which are quasi-equivalences, because $\epsilon: D(\mathcal{A}) \stackrel{\sim}{\rightarrow} H^{0}(\mathcal{D})$ is an equivalence of triangulated categories. They induce DG functors

$$
\mathcal{S F}(\mathcal{A}) \stackrel{p^{*}}{\longleftarrow} \mathcal{S F}\left(\tau_{\leq 0} \mathcal{B}\right) \stackrel{i^{*}}{\longrightarrow} \mathcal{S F}(\mathcal{B})
$$

that are quasi-equivalences as well. We also have a DG functor $\Phi: \mathcal{D} \rightarrow \mathcal{M}$ od $-\mathcal{B}$ defined by the rule $\Phi(X)(B):=\operatorname{Hom}_{\mathcal{B}}(B, X)$, where $B \in \mathcal{B}$ and $X \in \mathcal{D}$. The DG functor induces a quasi-functor $\phi: \mathcal{D} \rightarrow \mathcal{S F}(\mathcal{B})$ (see Remark 1.1). Since, by construction, objects of $\mathcal{B}$ form a set of compact generators of $H^{0}(\mathcal{D}) \cong D(\mathcal{A})$, Proposition 1.17implies that $\phi$ is a quasi-equivalence too. Thus, we get the following chain of quasi-equivalences:

$$
\mathcal{S F}(\mathcal{A}) \stackrel{p^{*}}{\longleftarrow} \mathcal{S F}\left(\tau_{\leq 0} \mathcal{B}\right) \stackrel{i^{*}}{\longrightarrow} \mathcal{S F}(\mathcal{B}) \stackrel{\phi}{\longleftarrow} \mathcal{D}
$$

and the enhancement $\mathcal{D}$ of $D(\mathcal{A})$ is equivalent to the standard enhancement $\mathcal{S} \mathcal{F}(\mathcal{A})$. The case of the subcategory of perfect DG modules can be considered similarly. 
The following theorems are our main results.

Theorem 2.7. Let $\mathcal{A}$ be a small category which we consider as a $D G$ category and $L \subset D(\mathcal{A})$ be a localizing subcategory with the quotient functor $\pi: D(\mathcal{A}) \rightarrow D(\mathcal{A}) / L$ that has a right adjoint (Bousfield localization) $\mu$. Assume that the following conditions hold:

a) for every $Y \in \mathcal{A}$ the object $\pi\left(h^{Y}\right) \in D(\mathcal{A}) / L$ is compact;

b) for every $Y, Z \in \mathcal{A}$ we have $\operatorname{Hom}\left(\pi\left(h^{Y}\right), \pi\left(h^{Z}\right)[i]\right)=0$ when $i<0$.

Then the triangulated category $D(\mathcal{A}) / L$ has a unique enhancement.

As we mentioned in Remark 1.22 in the above theorem we actually work with a small version of $D(\mathcal{A})$; i.e., the category $D(\mathcal{A})$ stands for $D_{\mathbb{U}}(\mathcal{A})$ for a chosen universe $\mathbb{U}$ (see the Appendix).

Theorem 2.8. Let $\mathcal{A}$ be a small category which we consider as a DG category and $L \subset D(\mathcal{A})$ be a localizing subcategory that is generated by compact objects $L^{c}:=L \cap D(\mathcal{A})^{c}$. Assume that for the quotient functor $\pi: D(\mathcal{A}) \rightarrow D(\mathcal{A}) / L$ the following condition holds:

$$
\text { for every } Y, Z \in \mathcal{A} \text { we have } \operatorname{Hom}\left(\pi\left(h^{Y}\right), \pi\left(h^{Z}\right)[i]\right)=0 \quad \text { when } i<0 .
$$

Then the triangulated subcategory of compact objects $(D(\mathcal{A}) / L)^{c}$ has a unique enhancement.

Note that under the condition that subcategory $L$ is generated by compact objects $L^{c}:=L \cap D(\mathcal{A})^{c}$, the quotient $D(\mathcal{A}) / L$ has small Hom-sets by Theorem 1.21 (1) and, hence, the Bousfield localization functor $\mu: D(\mathcal{A}) / L \rightarrow D(\mathcal{A})$ exists by Proposition 1.19 a). In addition, in this case, property a) of Theorem 2.7 automatically holds by Theorem 1.21 (2); i.e., for all $Y \in \mathcal{A}$ the objects $\pi\left(h^{Y}\right) \in D(\mathcal{A}) / L$ are compact. We also note that Theorems 2.7 and 2.8 have more advanced and precise versions (see Theorems 5.4 and 6.4).

It will be shown in Section 7 that Theorem 2.7 implies the following corollary.

Theorem 2.9. Let $\mathcal{C}$ be a Grothendieck category. Assume that it has a set of small generators which are compact objects in the derived category $D(\mathcal{C})$. Then the derived category $D(\mathcal{C})$ has a unique enhancement.

This result can be applied to the category of quasi-coherent sheaves on a quasicompact and quasi-separated scheme. Let $X$ be a a quasi-compact and quasiseparated scheme over $k$. Denote by Qcoh $X$ the abelian categories of quasi-coherent sheaves on $X$. We say that the scheme $X$ has enough locally free sheaves if for any finitely presented sheaf $\mathcal{F}$ there is an epimorphism $\mathcal{E} \rightarrow \mathcal{F}$ with a locally free sheaf $\mathcal{E}$ of finite type.

Theorem 2.10. Let $X$ be a quasi-compact and separated scheme that has enough locally free sheaves. Then the derived category of quasi-coherent sheaves $D(\mathrm{Qcoh} X)$ has a unique enhancement.

For the particular case of quasi-projective schemes we obtain

Corollary 2.11. Let $X$ be a quasi-projective scheme over $k$. Then the derived category of quasi-coherent sheaves $D(\mathrm{Qcoh} X)$ has a unique enhancement.

Denote by $\operatorname{Perf}(X) \subset D(Q \operatorname{coh} X)$ the full subcategory of perfect complexes which coincides with the subcategory of compact objects there (see [N2, BvB]). In Section 7 we also show that the above Theorem 2.8 implies the following statement. 
Theorem 2.12. Let $X$ be a quasi-projective scheme over $k$. Then the triangulated category of perfect complexes $\operatorname{Perf}(X)=D(\mathrm{Qcoh} X)^{c}$ has a unique enhancement.

In Section 8 we introduce a notion of compactly approximated objects in a triangulated category and we prove that the triangulated category of compactly approximated objects $(D(\mathcal{A}) / L)^{c a}$ has a unique enhancement (Theorem 8.8). This result allows us to deduce the uniqueness of enhancement for the bounded derived category of coherent sheaves on a quasi-projective scheme.

Theorem 2.13. The bounded derived category of coherent sheaves $D^{b}(\operatorname{coh} X)$ on a quasi-projective scheme $X$ has a unique enhancement.

In the case of projective varieties using results of [01, $\mathrm{O} 2$ we can prove a stronger result.

Theorem 2.14. Let $X$ be a projective scheme over $k$ such that the maximal torsion subsheaf $T_{0}\left(\mathcal{O}_{X}\right) \subset \mathcal{O}_{X}$ of dimension 0 is trivial. Then the triangulated categories $D^{b}(\operatorname{coh} X)$ and $\operatorname{Perf}(X)$ have strongly unique enhancements.

These results on uniqueness of enhancements also allow us to obtain some corollaries on the representation of fully faithful functors between derived categories of (quasi)-coherent sheaves.

Corollary 2.15. Let $X$ and $Y$ be quasi-compact separated schemes over a field $k$. Assume that $X$ has enough locally free sheaves. Let $F: D(\mathrm{Q} \operatorname{coh} X) \rightarrow D(\mathrm{Q} \operatorname{coh} Y)$ be a fully faithful functor that commutes with direct sums. Then there is an object $\mathcal{E} \cdot \in D(Q \operatorname{coh}(X \times Y))$ such that the functor $\Phi_{\mathcal{E}}$ is fully faithful and $\Phi_{\mathcal{E}} \cdot\left(C^{\cdot}\right) \cong$ $F\left(C^{\cdot}\right)$ for any $C^{\cdot} \in D(\mathrm{Q} \operatorname{coh} X)$.

Corollary 2.16. Let $X$ be a quasi-projective scheme and $Y$ be a quasi-compact and separated scheme. Let $K: \operatorname{Perf}(X) \rightarrow D(\mathrm{Qcoh} Y)$ be a fully faithful functor. Then there is an object $\mathcal{E} \in D(\mathrm{Q} \operatorname{coh}(X \times Y))$ such that

(1) the functor $\left.\Phi_{\mathcal{E}} \cdot\right|_{\operatorname{Perf}(X)}: \operatorname{Perf}(X) \rightarrow D(\mathrm{Qcoh} Y)$ is fully faithful and $\Phi_{\mathcal{E}} \cdot\left(P^{\cdot}\right)$ $\cong K\left(P^{\cdot}\right)$ for any $P^{\cdot} \in \operatorname{Perf}(X)$

(2) if $X$ is projective with $T_{0}\left(\mathcal{O}_{X}\right)=0$, then $\left.\Phi_{\mathcal{E}} \cdot\right|_{\operatorname{Perf}(X)} \cong K$;

(3) if $K$ sends $\operatorname{Perf}(X)$ to $\operatorname{Perf}(Y)$, then the functor $\Phi_{\mathcal{E}}: D(\mathrm{Q} c o h X) \rightarrow$ $D(\mathrm{Qcoh} Y)$ is fully faithful and also sends $\operatorname{Perf}(X)$ to $\operatorname{Perf}(Y)$;

(4) if $Y$ is Noetherian and $K$ sends $\operatorname{Perf}(X)$ to $D^{b}(\mathrm{Qcoh} Y)_{\mathrm{coh}}$, then the object $\mathcal{E} \cdot$ is isomorphic to an object of $D^{b}(\operatorname{coh}(X \times Y))$.

Corollary 2.17. Let $X$ be a projective scheme with $T_{0}\left(\mathcal{O}_{X}\right)=0$ and $Y$ be a quasi-compact and separated scheme. Let $K: D^{b}(\operatorname{coh} X) \rightarrow D(\mathrm{Q} \operatorname{coh} Y)$ be a fully faithful functor that commutes with homotopy limits. Then there is an object $\mathcal{E} \in$ $D(\mathrm{Q} \operatorname{coh}(X \times Y))$ such that $\left.\Phi_{\mathcal{E}} \cdot\right|_{D^{b}(\operatorname{coh} X)} \cong K$.

\section{Preliminary lemmas and Propositions}

The following four Sections 3-6 are devoted to proofs of two main Theorems 2.7 and 2.8. In this section we give some preliminary lemmas and present the main technical tool for the next sections, which is Proposition 3.4. In Section 4 we construct a quasi-functor $\widetilde{\rho}$ (formula (4.2)) which is a central object for all our considerations. In Sections 5 and 6 we give proofs of our main theorems. We show that we can apply the Drinfeld Theorem 1.3 to the quasi-functor $\widetilde{\rho}$ and argue 
that the induced quasi-functor $\rho$ is actually a quasi-equivalence between different enhancements.

Let $\mathcal{A}$ be a small category which we consider as a DG category. As above we denote by $\mathcal{M}$ od $-\mathcal{A}$ the $\mathrm{DG}$ category of DG $\mathcal{A}$-modules. Let us consider the full DG subcategory $\mathcal{S} \mathcal{F}(\mathcal{A}) \subset \mathcal{M}$ od $-\mathcal{A}$ of semi-free DG modules. Since $\mathcal{A}$ is an ordinary category, any semi-free $\mathrm{DG}$ module $P \in \mathcal{S F}(\mathcal{A})$ is actually a complex

$$
P=\left\{\cdots \longrightarrow P^{n-1} \longrightarrow P^{n} \longrightarrow \cdots\right\},
$$

where any $P^{n}=\bigoplus h^{Y}$ is a free $\mathcal{A}$-module $P$. Of course, not any such complex is a semi-free DG module. By definition, it is semi-free if it also has a filtration $0=\Phi_{0} \subset$ $\Phi_{1} \subset \ldots=P$ such that each quotient $\Phi_{i+1} / \Phi_{i}$ is a free DG $\mathcal{A}$-module. On the other hand, any bounded above such complex is semi-free. We denote by $\mathcal{S F}^{-}(\mathcal{A})$ the DG category of bounded above complexes of free $\mathcal{A}$-modules and denote by $\mathcal{S} \mathcal{F}_{f g}(\mathcal{A})$ the DG category of finitely generated semi-free DG modules, which is actually DG equivalent to the pretriangulated hull $\mathcal{A}^{\text {pre-tr }}$ of $\mathcal{A}$. We also denote by $\mathcal{P e r} f(\mathcal{A})$ the DG category of perfect DG modules, i.e. the full DG subcategory of $\mathcal{S} \mathcal{F}(\mathcal{A})$ consisting of all DG modules which are homotopy equivalent to a direct summand of a finitely generated semi-free DG module. Since any cohomologically bounded complex has a bounded above free resolution, the DG subcategory $\operatorname{Per} f(\mathcal{A}) \cap$ $\mathcal{S F}^{-}(\mathcal{A})$ is DG equivalent to $\operatorname{Per} f(\mathcal{A})$. So we will consider $\operatorname{Per} f(\mathcal{A})$ as a full DG subcategory of $\mathcal{S F}^{-}(\mathcal{A})$.

For any semi-free DG module $P$ we can consider the "stupid" truncations of $P$ that by definition are complexes of the form

$$
\begin{aligned}
\sigma_{\leq m} P & =\left\{\cdots \longrightarrow P^{m-1} \longrightarrow P^{m} \longrightarrow 0\right\}, \\
\sigma_{\geq n} P & =\left\{0 \longrightarrow P^{n} \longrightarrow P^{n+1} \longrightarrow \cdots\right\} .
\end{aligned}
$$

We also put

$$
P^{[n, m]}:=\sigma_{\geq n} \sigma_{\leq m} P=\left\{0 \longrightarrow P^{n} \longrightarrow \cdots \longrightarrow P^{m} \longrightarrow 0\right\} .
$$

The DG $\mathcal{A}$-modules $\sigma_{\leq m} P, \sigma_{\geq n} P, P^{[n, m]}$ are also semi-free. For every $n$ there is an exact sequence in $Z^{0}(\mathcal{S F}(\mathcal{A}))$,

$$
0 \longrightarrow \sigma_{\geq(n+1)} P \longrightarrow P \longrightarrow \sigma_{\leq n} P \longrightarrow 0 .
$$

For a fixed $m$ we also have $\sigma_{\leq m} P \cong \operatorname{colim}_{n} P^{[n, m]}$ in $Z^{0}\left(\mathcal{S F}^{-}(\mathcal{A})\right)$ and hence $\sigma_{\leq m} P \cong \operatorname{hocolim}_{n} P^{[n, m]}$ in $H^{0}\left(\mathcal{S F}^{-}(\mathcal{A})\right)$.

Let $\mathcal{U} \subset \mathcal{S F}(\mathcal{A})$ be a full pretriangulated DG subcategory that contains $\mathcal{A}$ and if $P \in \mathcal{U}$, then $\mathcal{U}$ contains all stupid truncations $\sigma_{\leq m} P$ and $\sigma_{\geq m} P$ as well. Denote by $h^{\bullet}: \mathcal{A} \rightarrow H^{0}(\mathcal{U})$ the natural fully faithful functor.

Let $F: H^{0}(\mathcal{U}) \rightarrow \mathcal{T}$ be an exact functor to a triangulated category $\mathcal{T}$ that has the following properties:

1) $F$ preserves all direct sums that exist in $H^{0}(\mathcal{U})$;

(*) 2) $\quad F\left(h^{Y}\right)$ is compact in $\mathcal{T}$ for every $Y \in \mathcal{A}$;

3) $\operatorname{Hom}\left(F\left(h^{Y}\right), F\left(h^{Z}\right)[s]\right)=0$ for every $Y, Z \in \mathcal{A}$ when $s<0$.

Remark 3.1. Since $F$ commutes with all direct sums that exist in $H^{0}(\mathcal{U})$, then it also commutes with any homotopy colimits that exist in $H^{0}(\mathcal{U})$.

Lemma 3.2. Let $\mathcal{U}$ be either $\mathcal{S} \mathcal{F}(\mathcal{A})$ or $\mathcal{S F}_{f g}(\mathcal{A})$, let $\mathcal{T}$ be a triangulated category and let $F: H^{0}(\mathcal{U}) \rightarrow \mathcal{T}$ be an exact functor. Assume that $F$ has the properties 
1), 2), 3) of (*) above. Then for any $Y \in \mathcal{A}$ and for any semi-free $D G \mathcal{A}$-module $P \in \mathcal{U}$ we have

$$
\operatorname{Hom}\left(F\left(h^{Y}\right), F\left(\sigma_{\geq n} P\right)[i]\right)=0
$$

when $i<n$.

Proof. The filtration on the semi-free DG module $P$ induces a filtration $\Phi_{j}^{\prime}:=$ $\Phi_{j} \cap \sigma_{\geq n} P$ on $\sigma_{\geq n} P$ and each quotient $\Phi_{j+1}^{\prime} / \Phi_{j}^{\prime}$ is isomorphic to a direct sum of objects $h^{Z}[s]$ with $s \leq-n$. The assumptions 1), 2), and 3) imply that

$$
\begin{aligned}
\operatorname{Hom}\left(F\left(h^{Y}\right), F\left(\Phi_{j+1}^{\prime} / \Phi_{j}^{\prime}\right)[i]\right) & =\operatorname{Hom}\left(F\left(h^{Y}\right), F\left(\bigoplus h^{Z}[s]\right)[i]\right) \\
& \cong \operatorname{Hom}\left(F\left(h^{Y}\right), \bigoplus F\left(h^{Z}[s+i]\right)\right) \\
& \cong \bigoplus \operatorname{Hom}\left(F\left(h^{Y}\right), F\left(h^{Z}\right)[s+i]\right)=0 .
\end{aligned}
$$

Hence, by induction, $\operatorname{Hom}\left(F\left(h^{Y}\right), F\left(\Phi_{j}^{\prime}\right)[i]\right)=0$ for all $j$.

In the case $\mathcal{U}=\mathcal{S F}_{f g}(\mathcal{A})$ everything is proved, because $\sigma_{\geq n} P \cong \Phi_{j}^{\prime}$ for some $j$.

In the case $\mathcal{U}=\mathcal{S F}(\mathcal{A})$ the object $\sigma_{\geq n} P$ is isomorphic to hocolim $\Phi_{j}^{\prime}$ in $H^{0}(\mathcal{S F}(\mathcal{A}))$. The functor $F$ preserves all direct sums; hence it preserves homotopy colimits and we get

$$
F\left(\sigma_{\geq n} P\right) \cong \underset{\operatorname{hocolim}}{\longrightarrow} F\left(\Phi_{j}^{\prime}\right) .
$$

Since the object $F\left(h^{Y}\right)$ is compact, the functor $\operatorname{Hom}\left(F\left(h^{Y}\right),-\right)$ commutes with direct sums and carries homotopy colimits to colimits of abelian groups (see [N1], Lemma 1.5). Thus

$$
\operatorname{Hom}\left(F\left(h^{Y}\right), F\left(\sigma_{\geq n} P\right)[i]\right)=\underline{\operatorname{colim}} \operatorname{Hom}\left(F\left(h^{Y}\right), F\left(\Phi_{j}^{\prime}\right)[i]\right)=0 .
$$

Corollary 3.3. Under the assumptions of Lemma 3.2 for every $Y \in \mathcal{A}$ and every $m \geq 0$ we have an injection

$\operatorname{Hom}\left(F\left(h^{Y}\right), F(P)\right) \hookrightarrow \operatorname{Hom}\left(F\left(h^{Y}\right), F\left(\sigma_{\leq m} P\right)\right) \cong \operatorname{colim}_{n} \operatorname{Hom}\left(F\left(h^{Y}\right), F\left(P^{[n, m]}\right)\right)$, which is a bijection when $m>0$.

Proof. The isomorphism is a consequence of the facts that $F$ preserves homotopy colimits and $F\left(h^{Y}\right)$ is compact. The injection follows from the exact triangle

$$
\sigma_{\geq(m+1)} P \rightarrow P \rightarrow \sigma_{\leq m} P
$$

and the last lemma which gives that $\operatorname{Hom}\left(F\left(h^{Y}\right), F\left(\sigma_{\geq(m+1)} P\right)\right)=0$ for $m \geq 0$. If $m>0$ we immediately obtain that the injection is also a bijection.

Proposition 3.4. Let $\mathcal{U}$ be either $\mathcal{S F}^{-}(\mathcal{A})$ or $\operatorname{Perf}(\mathcal{A})$ or $\mathcal{S F}_{\text {fg }}(\mathcal{A})$ and let $\mathcal{T}$ be a triangulated category. Let $F_{1}, F_{2}: H^{0}(\mathcal{U}) \rightarrow \mathcal{T}$ be two exact functors that satisfy conditions $(*)$. Assume that there is an isomorphism of functors $\theta: F_{1} \cdot h^{\bullet} \stackrel{\sim}{\rightarrow} F_{2} \cdot h^{\bullet}$ from $\mathcal{A}$ to $\mathcal{T}$. Then for every $P \in \mathcal{U}$ there exists an isomorphism $\theta_{P}: F_{1}(P) \stackrel{\sim}{\rightarrow}$ $F_{2}(P)$ such that for any $Y \in \mathcal{A}$ and every $f \in \operatorname{Hom}_{H^{0}(\mathcal{U})}\left(h^{Y}[-k], P\right)$ where $k \in \mathbb{Z}$ the diagram

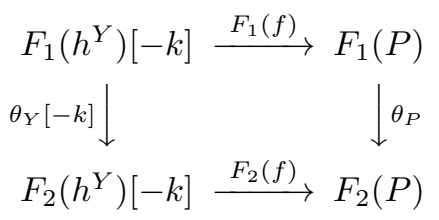

commutes in $\mathcal{T}$. 
Proof. We will construct $\theta_{P}$ in a few steps.

Step 1 . Denote by $h^{\bullet}(\mathcal{A})^{\oplus} \subset H^{0}(\mathcal{U})$ the full subcategory of $H^{0}(\mathcal{U})$ which is obtained from $h^{\bullet}(\mathcal{A})$ by adding arbitrary (existing in $H^{0}(\mathcal{U})$ ) direct sums of objects of $h^{\bullet}(\mathcal{A})$.

Since the functors $F_{i}$ commute with direct sums we can extend our transformation $\theta$ onto the whole subcategory $h^{\bullet}(\mathcal{A})^{\oplus}$. Indeed, for any $P=\bigoplus h^{Y}$ the canonical isomorphisms $\bigoplus F_{i}\left(h^{Y}\right) \cong F_{i}\left(\bigoplus h^{Y}\right)$ allow us to define an isomorphism $\theta_{P}: F_{1}\left(\bigoplus h^{Y}\right) \rightarrow F_{2}\left(\bigoplus h^{Y}\right)$ as the product of the canonical morphisms $F_{1}\left(h^{Y}\right) \stackrel{\theta_{Y}}{\rightarrow} F_{2}\left(h^{Y}\right) \rightarrow F_{2}\left(\bigoplus h^{Y}\right)$.

Step 2. Now consider a semi-free DG module $P=\left\{\cdots \rightarrow P^{m-1} \rightarrow P^{m}\right\}$ which by assumption is bounded above. For every $n \leq m$ we have the exact triangle in $H^{0}(\mathcal{U})$ :

$$
P^{n-1}[-n] \longrightarrow \sigma_{\geq n} P \longrightarrow \sigma_{\geq(n-1)} P .
$$

For all $P^{k}$ we have the isomorphisms $\theta^{k}: F_{1}\left(P^{k}\right) \stackrel{\sim}{\rightarrow} F_{2}\left(P^{k}\right)$ constructed in Step 1 .

In Step 2 by descending induction on $n \leq m$ we will construct isomorphisms $\theta_{\geq n}: F_{1}\left(\sigma_{\geq n} P\right) \stackrel{\sim}{\rightarrow} F_{2}\left(\sigma_{\geq n} P\right)$ such that there is an isomorphism of triangles

$$
\begin{array}{crrr}
F_{1}\left(P^{n-1}\right)[-n] & F_{1}\left(\sigma_{\geq n} P\right) & \longrightarrow F_{1}\left(\sigma_{\geq(n-1)} P\right) & \longrightarrow F_{1}\left(P^{n-1}\right)[-n+1] \\
\left.\theta^{n-1}[-n]\right\rfloor & \mid \theta_{\geq(n-1)} & \downarrow \theta^{n-1}[-n+1] \\
F_{2}\left(P^{n-1}\right)[-n] & \longrightarrow F_{2}\left(\sigma_{\geq n} P\right) & \longrightarrow F_{2}\left(\sigma_{\geq(n-1)} P\right) & \longrightarrow F_{2}\left(P^{n-1}\right)[-n+1]
\end{array}
$$

for all $n \leq m$.

We start with the diagram

$$
\begin{array}{ccc}
F_{1}\left(P^{m-1}[-m]\right) & F_{1}\left(P^{m}[-m]\right) \\
\theta^{m-1}[-m] \downarrow & \downarrow \theta^{m}[-m] & \\
F_{2}\left(P^{m-1}[-m]\right) & \left.\longrightarrow F_{2}\left(P^{m}[-m]\right) \longrightarrow \sigma_{\geq(m-1)} P\right) \\
& \longrightarrow F_{2}\left(\sigma_{\geq(m-1)} P\right),
\end{array}
$$

where the square is commutative. Hence, there exists an isomorphism $\theta_{\geq(m-1)}$ : $F_{1}\left(\sigma_{\geq(m-1)} P\right) \rightarrow F_{2}\left(\sigma_{\geq(m-1)} P\right)$ which completes the above diagram to a morphism of triangles:

$$
\begin{array}{crrrr}
F_{1}\left(P^{m-1}[-m]\right) & \rightarrow F_{1}\left(P^{m}[-m]\right) & \rightarrow F_{1}\left(\sigma_{\geq(m-1)} P\right) & \rightarrow & F_{1}\left(P^{m-1}[-m+1]\right) \\
\left.\theta^{m-1}[-m]\right\rfloor & \downarrow \theta^{m}[-m] & \downarrow \theta_{\geq(m-1)} & & \downarrow \theta^{m-1}[-m+1] \\
F_{2}\left(P^{m-1}[-m]\right) & \rightarrow F_{2}\left(P^{m}[-m]\right) & \rightarrow F_{2}\left(\sigma_{\geq(m-1)} P\right) & \rightarrow & F_{2}\left(P^{m-1}[-m+1]\right) .
\end{array}
$$

This provides the base of induction.

Assume by induction that we have defined isomorphisms $\theta_{\geq(n+1)}, \theta_{\geq n}$ so that the diagram

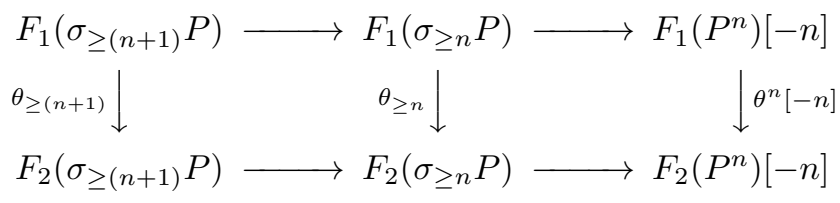


is a morphism of triangles. Then we claim that the natural diagram

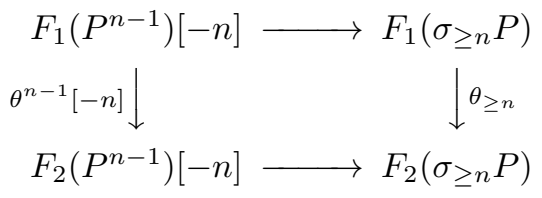

commutes. Indeed, complete it to a diagram

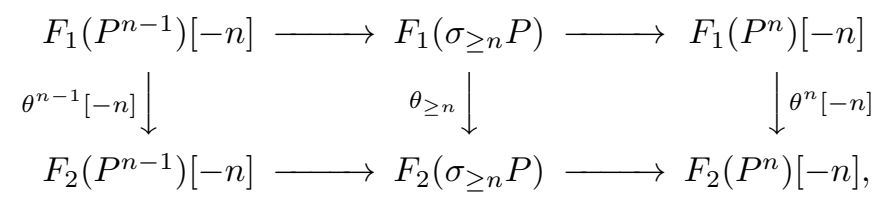

where the right square and the outside square commute. By Corollary 3.3 the natural map

$$
\operatorname{Hom}\left(F_{1}\left(P^{n-1}\right)[-n], F_{1}\left(\sigma_{\geq n} P\right)\right) \longrightarrow \operatorname{Hom}\left(F_{1}\left(P^{n-1}\right)[-n], F_{1}\left(P^{n}\right)[-n]\right)
$$

is injective. Using the compositions with the isomorphisms $\theta_{\geq n}$ and $\theta^{n}[-n]$ we conclude that the natural map

$$
\operatorname{Hom}\left(F_{1}\left(P^{n-1}\right)[-n], F_{2}\left(\sigma_{\geq n} P\right)\right) \longrightarrow \operatorname{Hom}\left(F_{1}\left(P^{n-1}\right)[-n], F_{2}\left(P^{n}\right)[-n]\right)
$$

is also injective. Therefore the left square in the above diagram commutes too. Hence, there is an isomorphism $\theta_{\geq(n-1)}: F_{1}\left(\sigma_{\geq(n-1)} P\right) \rightarrow F_{2}\left(\sigma_{\geq(n-1)} P\right)$ such that the diagram

$$
\begin{array}{crrr}
F_{1}\left(P^{n-1}\right)[-n] & \longrightarrow F_{1}\left(\sigma_{\geq n} P\right) & \longrightarrow F_{1}\left(\sigma_{\geq(n-1)} P\right) & \longrightarrow F_{1}\left(P^{n-1}\right)[-n+1] \\
\left.\theta^{n-1}[-n]\right\rfloor & \theta_{\geq n} \downarrow & \theta_{\geq(n-1)} & \downarrow \theta^{n-1}[-n+1] \\
F_{2}\left(P^{n-1}\right)[-n] & \longrightarrow F_{2}\left(\sigma_{\geq n} P\right) & \longrightarrow F_{2}\left(\sigma_{\geq(n-1)} P\right) & \longrightarrow F_{2}\left(P^{n-1}\right)[-n+1]
\end{array}
$$

is an isomorphism of triangles. This completes the induction step.

If $\mathcal{U}=\mathcal{S F}_{f g}(\mathcal{A})$, the construction of isomorphisms $\theta_{P}$ is finished.

Step 3. Let us now consider the case $\mathcal{U}=\mathcal{S F}^{-}(\mathcal{A})$. Since $P$ is bounded above we have a natural isomorphism $\operatorname{colim}_{n}\left(\sigma_{\geq n} P\right) \stackrel{\sim}{\rightarrow} P$ in $Z^{0}\left(\mathcal{S F}^{-}(\mathcal{A})\right)$ and hence $P$ is isomorphic to ${\underset{\text { hocolim}}{\longrightarrow}}_{n}\left(\sigma_{\geq n} P\right)$ in $H^{0}\left(\mathcal{S F}^{-}(\mathcal{A})\right)$. The canonical sequence

$$
\bigoplus_{n \leq m}\left(\sigma_{\geq n} P\right) \longrightarrow \bigoplus_{n \leq m}\left(\sigma_{\geq n} P\right) \longrightarrow P
$$

gives an exact triangle in $H^{0}(\mathcal{S F}(\mathcal{A}))$.

Since the functors $F_{i}$ commute with direct sums we can extend our isomorphisms $\theta_{\geq n}$ on the direct sum. Indeed, for the object $\bigoplus\left(\sigma_{\geq n} P\right)$ the canonical isomorphism $\bigoplus F_{i}\left(\sigma_{\geq n} P\right) \cong F_{i}\left(\bigoplus \sigma_{\geq n} P\right)$ allows us to define an isomorphism $\theta_{\oplus}: F_{1}\left(\bigoplus \sigma_{\geq n} P\right) \rightarrow F_{2}\left(\bigoplus \sigma_{\geq n} P\right)$ as the product of the canonical morphisms $F_{1}\left(\sigma_{\geq n} P\right) \stackrel{\theta_{\geq n}}{\longrightarrow} F_{2}\left(\sigma_{\geq n} P\right) \stackrel{\text { can }}{\longrightarrow} F_{2}\left(\bigoplus \sigma_{\geq n} P\right)$. Now there is an isomorphism $\theta_{P}$ : $F_{1}(P) \stackrel{\sim}{\rightarrow} F_{2}(P)$ that gives an isomorphism of triangles

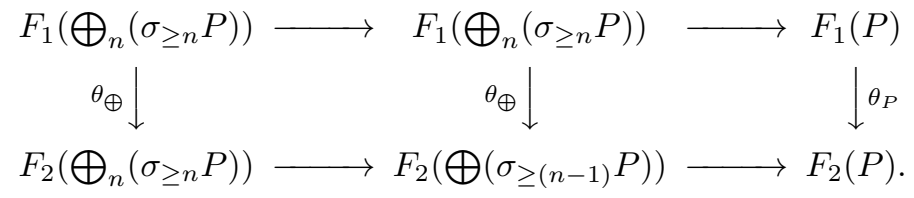


It is easy to see that the isomorphism $\theta_{P}: F_{1}(P) \stackrel{\sim}{\rightarrow} F_{2}(P)$ makes the following diagrams commutative for each $n$ :

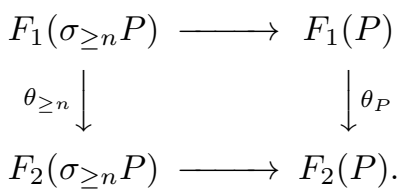

Step 4. Let us now consider the case $\mathcal{U}=\operatorname{Perf}(\mathcal{A}) \subset \mathcal{S F}^{-}(\mathcal{A})$. By definition, the object $P \in \mathcal{P} \operatorname{erf}(\mathcal{A})$ is a direct summand of a finitely generated object $Q \in$ $\mathcal{S} \mathcal{F}_{f g}(\mathcal{A})$ in the triangulated category $H^{0}(\mathcal{U}) \subset D(\mathcal{A})$. Take a sufficiently negative $n \ll 0$. The object $\sigma_{\geq n} P$ is finitely generated semi-free and, hence, the object $\sigma_{\leq(n-1)} P$ is a perfect DG module as well. Thus it is homotopy equivalent to a direct summand of a finitely generated semi-free DG module $R$. It is easy to see that the object $\sigma_{\leq(n-1)} P$ is also a direct summand of the finitely generated DG module $\sigma_{\leq(n-1)} R$. Let us consider a diagram

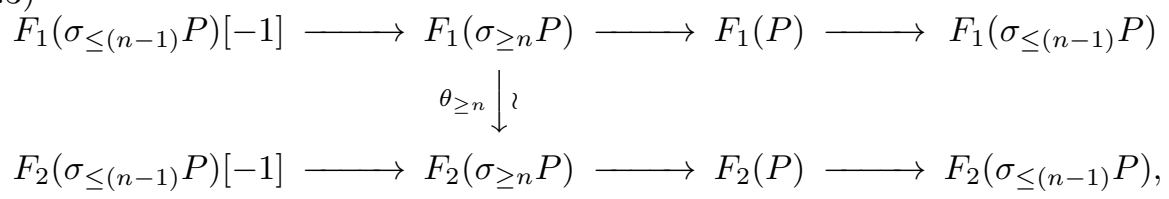

where $\theta_{\geq n}$ was constructed in Step 2. Since $n \ll 0$ and $\sigma_{\leq(n-1)} R, Q \in \mathcal{S F}_{f g}(\mathcal{A})$ we obtain that

$$
\operatorname{Hom}\left(F_{j}\left(\sigma_{\leq(n-1)} R\right)[i], F_{k}(Q)\right)=0 \quad \text { for all } \quad i \geq-1, \quad \text { and } \quad j, k=1,2
$$

by Lemma 3.2. This implies that for their direct summands $\sigma_{\leq(n-1)} P$ and $P$ we also have

$$
\operatorname{Hom}\left(F_{j}\left(\sigma_{\leq(n-1)} P\right)[-1], F_{k}(P)\right)=0 \quad \text { for all } \quad i \geq-1, \quad \text { and } \quad j, k=1,2 .
$$

Therefore, there are unique morphisms

$$
\theta_{P}: F_{1}(P) \rightarrow F_{2}(P) \quad \text { and } \quad \theta_{\leq(n-1)}: F_{1}\left(\sigma_{\leq(n-1)} P\right) \rightarrow F_{2}\left(\sigma_{\leq(n-1)} P\right)
$$

that complete the diagram (3.3) to a morphism of triangles. Since $\theta_{\geq n}$ is an isomorphism, the cones $C\left(\theta_{P}\right)$ and $C\left(\theta_{\leq(n-1)}\right)$ are isomorphic. The vanishing conditions (3.4) imply that

$$
\operatorname{Hom}\left(F_{2}\left(\sigma_{\leq(n-1)} P\right), C\left(\theta_{P}\right)\right)=0 \quad \text { and } \operatorname{Hom}\left(F_{1}\left(\sigma_{\leq(n-1)} P\right)[1], C\left(\theta_{P}\right)\right)=0 .
$$

Hence, the cone $C\left(\theta_{\leq(n-1)}\right) \cong C\left(\theta_{P}\right)$ is trivial and $\theta_{P}$ is an isomorphism.

Finally, since for any $n \ll 0$ the isomorphism $\theta_{P}$ is unique, it does not depend on $n$. More precisely, for any $n \ll 0$ and each $k>n$ the right and the left squares in the diagram

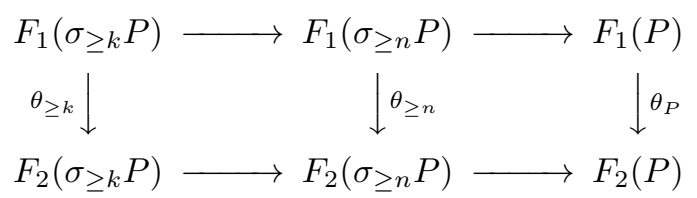

are commutative. Hence the outside square is also commutative for each $k \in \mathbb{Z}$. 
Step 5. Now we should check that the diagram (3.1)

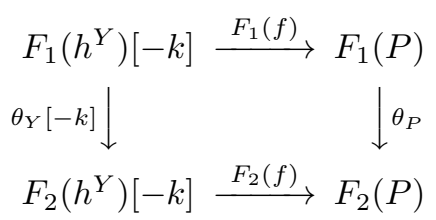

commutes for any $Y \in \mathcal{A}$ and every $f \in \operatorname{Hom}_{H^{0}(\mathcal{U})}\left(h^{Y}[-k], P\right)$.

The map $f$ is represented by a morphism of the complexes $f: h^{Y}[-k] \rightarrow P$. It is decomposed as

$$
h^{Y}[-k] \rightarrow \sigma_{\geq k} P \rightarrow P .
$$

In the diagram

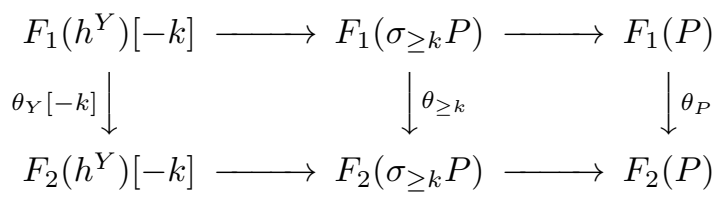

the right square commutes for any $k \in \mathbb{Z}$ by the construction of $\theta_{P}$. Thus it is sufficient to prove that the first square commutes too. The map $h^{Y}[-k] \rightarrow \sigma_{\geq k} P \rightarrow$ $P$ is induced by a map $f_{k}: h^{Y} \rightarrow P^{k}$. Consider the diagram

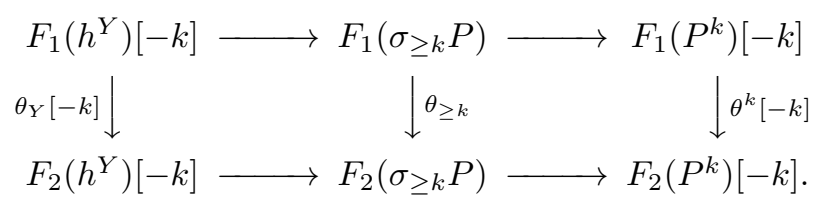

The right square commutes by (3.2) and the outside square commutes by Step 1 .

By Corollary 3.3 the natural map

$$
\operatorname{Hom}\left(F_{1}\left(h^{Y}\right)[-k], F_{1}\left(\sigma_{\geq k} P\right)\right) \longrightarrow \operatorname{Hom}\left(F_{1}\left(h^{Y}\right)[-k], F_{1}\left(P^{k}\right)[-k]\right)
$$

is injective. Using the compositions with the isomorphisms $\theta_{\geq k}$ and $\theta^{k}[-k]$ we conclude that the natural map

$$
\operatorname{Hom}\left(F_{1}\left(h^{Y}\right)[-k], F_{2}\left(\sigma_{\geq k} P\right)\right) \longrightarrow \operatorname{Hom}\left(F_{1}\left(h^{Y}\right)[-k], F_{2}\left(P^{k}\right)[-k]\right)
$$

is also injective. Therefore the left square in the above diagram commutes too.

\section{Preliminary constructions}

Let $\mathcal{A}$ be a small category which we consider as a DG category and $L \subset D(\mathcal{A})$ be a localizing subcategory with the quotient functor $\pi: D(\mathcal{A}) \rightarrow D(\mathcal{A}) / L$.

As above we denote by $\mathcal{M} o d-\mathcal{A}$ the DG category of DG $\mathcal{A}$-modules. Consider the DG subcategories $\mathcal{S F}(\mathcal{A}) \subset \mathcal{P}(\mathcal{A}) \subset \mathcal{M o d}-\mathcal{A}$ of semi-free and h-projective DG modules, respectively. The canonical DG functors $\mathcal{S F}(\mathcal{A}) \hookrightarrow \mathcal{P}(\mathcal{A}) \hookrightarrow \mathcal{M}$ od $-\mathcal{A} /$ $\mathcal{A} c(\mathcal{A})$ are quasi-equivalences and give equivalent enhancements of the derived category $D(\mathcal{A})$ (see Example 2.5). As a consequence, the category $D(\mathcal{A}) / L$ has canonical enhancements. Namely, if $\mathcal{L} \subset \mathcal{M o d}-\mathcal{A}$ is the full DG subcategory having the same objects as $L$, then the natural DG functors

$$
\mathcal{S F}(\mathcal{A}) / \mathcal{L} \cap \mathcal{S F}(\mathcal{A}) \longrightarrow \mathcal{P}(\mathcal{A}) / \mathcal{L} \cap \mathcal{P}(\mathcal{A}) \longrightarrow \mathcal{M o d}-\mathcal{A} / \mathcal{L}
$$


are quasi-equivalences; i.e., the canonical functors

$H^{0}(\mathcal{S F}(\mathcal{A}) / \mathcal{L} \cap \mathcal{S F}(\mathcal{A})) \stackrel{\sim}{\longrightarrow} H^{0}(\mathcal{P}(\mathcal{A}) / \mathcal{L} \cap \mathcal{P}(\mathcal{A})) \stackrel{\sim}{\longrightarrow} H^{0}(\mathcal{M o d}-\mathcal{A} / \mathcal{L}) \cong D(\mathcal{A}) / L$ are equivalences.

Denote by $\tilde{\pi}$ the quotient DG functor $\tilde{\pi}: \mathcal{S F}(\mathcal{A}) \rightarrow \mathcal{S F}(\mathcal{A}) / \mathcal{L} \cap \mathcal{S F}(\mathcal{A})$. We denote the composition of the functor $H^{0}\left(h^{\bullet}\right): \mathcal{A} \rightarrow H^{0}(\mathcal{M}$ od $-\mathcal{A})$ with the localization $H^{0}(\mathcal{M o d}-\mathcal{A}) \rightarrow D(\mathcal{A})$ simply by $h^{\bullet}$. Note that this functor $h^{\bullet}: \mathcal{A} \rightarrow D(\mathcal{A})$ is full and faithful.

Consider an enhancement of the triangulated category of compact objects $(D(\mathcal{A}) / L)^{c}$. This means that there are given a pretriangulated DG category $\mathcal{C}$ and an equivalence of triangulated categories $\epsilon:(D(\mathcal{A}) / L)^{c} \stackrel{\sim}{\rightarrow} H^{0}(\mathcal{C})$. Denote by $\mathcal{B} \subset \mathcal{C}$ the full DG subcategory with the set of objects $\left\{\epsilon \pi\left(h^{Y}\right)\right\}_{Y \in \mathcal{A}}$. As in formula (1.1) before Proposition 1.16 there is the canonical DG functor

$$
\Psi: \mathcal{C} \longrightarrow \mathcal{M o d}-\mathcal{B}, \quad \text { where } \Psi(X)(B)=\operatorname{Hom}_{\mathcal{C}}(Y, X) \quad \text { for } \quad B \in \mathcal{B}, X \in \mathcal{C} .
$$

In composition with the DG functor $\operatorname{Mod}-\mathcal{B} \rightarrow \mathcal{M o d}-\mathcal{B} / \mathcal{A} c(\mathcal{B})$ it induces (as in Remark 1.1) a quasi-functor

$$
\psi: \mathcal{C} \longrightarrow \mathcal{S F}(\mathcal{B}), \quad H^{0}(\psi): H^{0}(\mathcal{C}) \longrightarrow H^{0}(\mathcal{S F}(\mathcal{B})) \cong D(\mathcal{B}) .
$$

Since the objects $\left\{\pi\left(h^{Y}\right)\right\}_{Y \in \mathcal{A}}$ are compact in $D(\mathcal{A}) / L$ we have the following composition:

$$
\mathcal{A} \stackrel{\pi h \bullet}{\longrightarrow}(D(A) / L)^{c} \stackrel{\epsilon}{\longrightarrow} H^{0}(\mathcal{C}) \stackrel{H^{0}(\psi)}{\longrightarrow} D(\mathcal{B})
$$

By construction it factors through the canonical embedding $H^{0}(\mathcal{B}) \hookrightarrow \mathcal{D}(\mathcal{B})$ and we denote by $a$ the corresponding functor $a: \mathcal{A} \rightarrow H^{0}(\mathcal{B})$.

Denote by $\tau_{\leq 0} \mathcal{B}$ the DG subcategory with the same objects as $\mathcal{B}$ and morphisms

$$
\operatorname{Hom}_{\tau_{\leq 0} \mathcal{B}}(M, N)=\tau_{\leq 0} \operatorname{Hom}_{\mathcal{B}}(M, N),
$$

where $\tau_{\leq 0}$ is the usual truncation of complexes as in (2.1).

We have the obvious diagram of DG categories and DG functors

$$
\mathcal{A} \stackrel{a}{\longrightarrow} H^{0}(\mathcal{B}) \stackrel{p}{\longleftarrow} \tau_{\leq 0} \mathcal{B} \stackrel{i}{\longrightarrow} \mathcal{B}
$$

where the functor $p: \tau_{\leq 0} \mathcal{B} \rightarrow H^{0}(B)$ is a quasi-equivalence by condition b) in the assumptions of Theorem 2.7. They induce DG functors between semi-free DG modules, and we obtain a commutative diagram

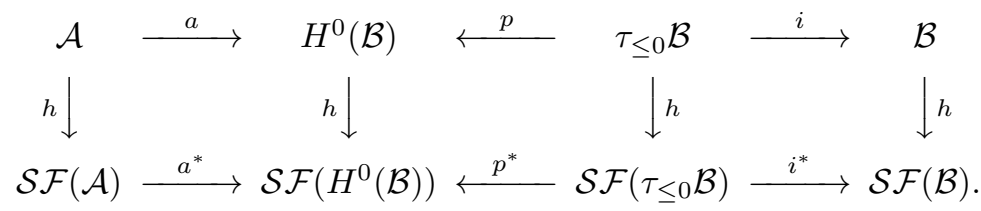

Passing to homotopy categories we get the following commutative diagram:

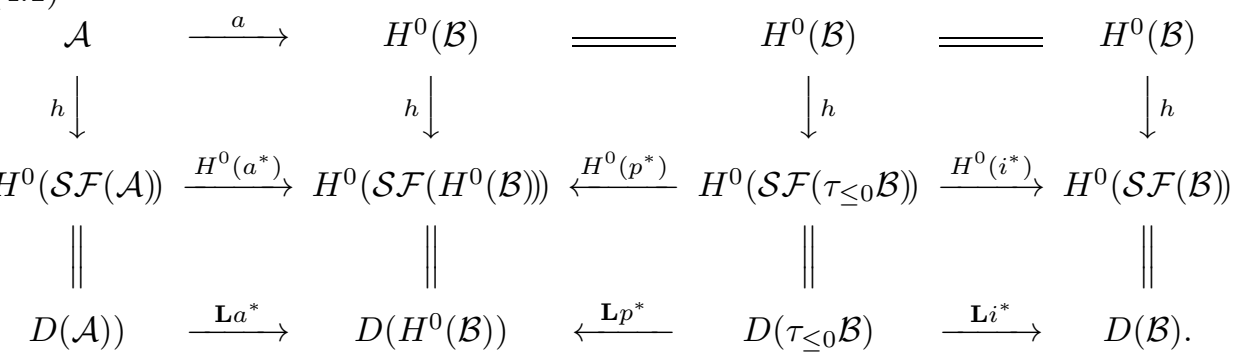


The DG functor $p$ and, hence, the DG functor $p^{*}$ are quasi-equivalences. This gives us a quasi-functor $p_{*}: \mathcal{S F}\left(H^{0}(\mathcal{B})\right) \rightarrow \mathcal{S F}\left(\tau_{\leq 0} \mathcal{B}\right)$. This also implies that the functor $H^{0}\left(p^{*}\right) \cong \mathbf{L} p^{*}$ is an equivalence and the right adjoint functor $p_{*}: D\left(H^{0}(\mathcal{B})\right) \rightarrow$ $D\left(\tau_{\leq 0} \mathcal{B}\right)$ is its quasi-inverse.

We denote by $\widetilde{\rho}$ the quasi-functor that is the composition

$$
\widetilde{\rho}: \mathcal{S F}(\mathcal{A}) \stackrel{a^{*}}{\longrightarrow} \mathcal{S F}\left(H^{0}(\mathcal{B})\right) \stackrel{p_{*}}{\longrightarrow} \mathcal{S F}\left(\tau_{\leq 0} \mathcal{B}\right) \stackrel{i^{*}}{\longrightarrow} \mathcal{S F}(\mathcal{B})
$$

and denote by $F_{1}$ the induced functor

$$
F_{1}=H^{0}(\widetilde{\rho}): D(\mathcal{A}) \stackrel{\mathbf{L} a^{*}}{\longrightarrow} D\left(H^{0}(\mathcal{B})\right) \stackrel{p_{*}}{\longrightarrow} D\left(\tau_{\leq 0} \mathcal{B}\right) \stackrel{\mathbf{L} i^{*}}{\longrightarrow} D(\mathcal{B}) .
$$

The functor $F_{1}$ evidently preserves direct sums.

\section{Proof of Theorem 2.7}

Let $\mathcal{D}$ be a pretriangulated DG category and $\widetilde{\epsilon}: D(\mathcal{A}) / L \stackrel{\sim}{\rightarrow} H^{0}(\mathcal{D})$ be an equivalence of triangulated categories. Denote by $\mathcal{C} \subset \mathcal{D}$ the full DG subcategory of compact objects in $H^{0}(\mathcal{D})$. The equivalence $\tilde{\epsilon}$ induces an equivalence $\epsilon:(D(\mathcal{A}) / L)^{c} \stackrel{\sim}{\rightarrow} H^{0}(\mathcal{C})$. Thus we can apply the construction from the previous section.

As above denote by $\mathcal{B} \subset \mathcal{C} \subset \mathcal{D}$ the full DG subcategory with the set of objects $\left\{\tilde{\epsilon} \pi\left(h^{Y}\right)\right\}_{Y \in \mathcal{A}}$. By construction in the previous section, formulas (4.2) and (4.3) give a quasi-functor

$$
\widetilde{\rho}: \mathcal{S F}(\mathcal{A}) \longrightarrow \mathcal{S F}(\mathcal{B})
$$

and the corresponding functor $F_{1}=H^{0}(\widetilde{\rho}): D(\mathcal{A}) \rightarrow D(\mathcal{B})$.

Notice that, by assumption, the objects $\left\{\widetilde{\epsilon} \pi\left(h^{Y}\right)\right\}_{Y \in \mathcal{A}}$ are compact and by Remark 1.20 they compactly generate the category $H^{0}(\mathcal{D})$. Therefore, by Proposition 1.17 the canonical DG functor $\Phi: \mathcal{D} \rightarrow \mathcal{M o d}-\mathcal{B}$, which is defined by formula (1.1) $\Phi(X)(B)=\operatorname{Hom}_{\mathcal{D}}(B, X)$ for $B \in \mathcal{B}, X \in \mathcal{D}$, induces a quasi-functor

$$
\phi: \mathcal{D} \rightarrow \mathcal{S F}(\mathcal{B}) \quad \text { such that } H^{0}(\phi): H^{0}(\mathcal{D}) \stackrel{\sim}{\longrightarrow} H^{0}(\mathcal{S F}(\mathcal{B})) \cong D(\mathcal{B})
$$

is an equivalence. Therefore, $\mathcal{S} \mathcal{F}(\mathcal{B})$ is another enhancement of $D(\mathcal{A}) / L$ which is equivalent to $\mathcal{D}$. Thus it is sufficient to prove that $\mathcal{S F}(\mathcal{B})$ is quasi-equivalent to $\mathcal{S F}(\mathcal{A}) / \mathcal{L} \cap \mathcal{S F}(\mathcal{A})$.

Denote by $F_{2}$ the composition of $H^{0}(\phi)$ with the equivalence $\widetilde{\epsilon}$ and the localization $\pi$,

$$
F_{2}: D(\mathcal{A}) \stackrel{\pi}{\longrightarrow} D(A) / L \stackrel{\tilde{\epsilon}}{\longrightarrow} H^{0}(\mathcal{D}) \stackrel{H^{0}(\phi)}{\longrightarrow} D(\mathcal{B}) .
$$

Thus we have two functors $F_{1}$ and $F_{2}$ from $D(\mathcal{A})$ to $D(\mathcal{B})$ and both of them enjoy properties 1$), 2), 3$ ) of $(*)$. By construction of $F_{2}$ and $a$, the composition of $h^{\bullet}: \mathcal{A} \rightarrow \mathcal{D}(\mathcal{A})$ and $F_{2}$ coincides with $\mathcal{A} \stackrel{a}{\rightarrow} H^{0}(\mathcal{B}) \rightarrow D(\mathcal{B})$. The commutativity of diagram (4.1) immediately proves the following lemma.

Lemma 5.1. There exists an isomorphism $\theta: F_{1} \cdot h^{\bullet} \stackrel{\sim}{\rightarrow} F_{2} \cdot h \bullet$ of functors from $\mathcal{A}$ to $D(\mathcal{B})$.

Now we are ready to prove the following lemma.

Lemma 5.2. The quasi-functor $\widetilde{\rho}: \mathcal{S F}(\mathcal{A}) \rightarrow \mathcal{S F}(\mathcal{B})$ factors through the $D G$ quotient $\mathcal{S} \mathcal{F}(\mathcal{A}) / \mathcal{L} \cap \mathcal{S F}(\mathcal{A})$. 
Proof. By the Drinfeld Theorem 1.3 it is sufficient to show that the corresponding functor $F_{1}: D(\mathcal{A}) \rightarrow D(\mathcal{B})$ factors through the quotient $D(\mathcal{A}) / L$.

Let $P \in \mathcal{S} \mathcal{F}(\mathcal{A})$ be a semi-free DG module that belongs to $\mathcal{L}$. Consider it as the object of $D(\mathcal{A})$. We have to show that $F_{1}(P) \cong 0$. Since $\left\{F_{1}\left(h^{Y}\right)\right\}_{Y \in \mathcal{A}}$ is a set of compact generators of $D(\mathcal{B})$ it is enough to check that for any $Y \in \mathcal{A}$ and any $k \in \mathbb{Z}$

$$
\operatorname{Hom}\left(F_{1}\left(h^{Y}\right)[k], F_{1}(P)\right)=0 .
$$

Let us consider a stupid truncation $\sigma_{\geq(m+1)} P \rightarrow P \rightarrow \sigma_{\leq m} P$, for some $m>k$.

By Corollary 3.3. we have an isomorphism

$$
\operatorname{Hom}\left(F_{1}\left(h^{Y}\right)[k], F_{1}(P)\right) \cong \operatorname{Hom}\left(F_{1}\left(h^{Y}\right)[k], F_{1}\left(\sigma_{\leq m} P\right)\right) .
$$

On the other hand, $\sigma_{\leq m} P$ belongs to $\mathcal{S F}^{-}(\mathcal{A})$ and by Proposition 3.4 there is an isomorphism $F_{1}\left(\sigma_{\leq m} \bar{P}\right) \cong F_{2}\left(\sigma_{\leq m} P\right)$. Now applying Corollary 3.3 to the functor $F_{2}$ we obtain isomorphisms

$$
\begin{aligned}
\operatorname{Hom}\left(F_{1}\left(h^{Y}\right)[k], F_{1}(P)\right) & \cong \operatorname{Hom}\left(F_{1}\left(h^{Y}\right)[k], F_{1}\left(\sigma_{\leq m} P\right)\right) \\
& \cong \operatorname{Hom}\left(F_{2}\left(h^{Y}\right)[k], F_{2}\left(\sigma_{\leq m} P\right)\right) \\
& \cong \operatorname{Hom}\left(F_{2}\left(h^{Y}\right)[k], F_{2}(P)\right)=0,
\end{aligned}
$$

where the last equality holds because $F_{2}(P)=H^{0}(\phi) \cdot \tilde{\epsilon} \cdot \pi(P)=0$.

Thus, by Theorem 1.3 there exists a quasi-functor

$$
\rho: \mathcal{S F}(\mathcal{A}) / \mathcal{L} \cap \mathcal{S F}(\mathcal{A}) \longrightarrow \mathcal{S F}(\mathcal{B})
$$

and an isomorphism of functors $F_{1}=H^{0}(\widetilde{\rho}) \cong H^{0}(\rho) \cdot \pi$.

Now the following lemma finishes the proof of the theorem.

Lemma 5.3. The functor $H^{0}(\rho): D(\mathcal{A}) / L \longrightarrow D(\mathcal{B})$ is an equivalence.

Proof. Let us prove that the functor $H^{0}(\rho)$ is fully faithful.

The set $\left\{\pi\left(h^{Y}\right)\right\}_{Y \in \mathcal{A}}$ is a set of compact generators for the category $D(\mathcal{A}) / L$, and the functor $H^{0}(\rho)$ preserves direct sums, since $\pi$ and $H^{0}(\widetilde{\rho})$ do. Thus, it suffices to prove that the map

$$
H^{0}(\rho): \operatorname{Hom}\left(\pi\left(h^{Y}\right)[k], \pi\left(h^{Z}\right)\right) \longrightarrow \operatorname{Hom}\left(H^{0}(\rho) \pi\left(h^{Y}\right)[k], H^{0}(\rho) \pi\left(h^{Z}\right)\right)
$$

is an isomorphism for every $Y, Z \in \mathcal{A}$ and any $k \in \mathbb{Z}$.

Let us fix $Y, Z$ and $k$ as above. Recall that the localization functor $\pi$ has a right adjoint functor $\mu: D(\mathcal{A}) / L \rightarrow D(\mathcal{A})$ which is full and faithful, so that the natural morphism of functors $\operatorname{id}_{D(\mathcal{A}) / L} \rightarrow \pi \mu$ is an isomorphism. Let $P \cong \mu \pi\left(h^{Z}\right) \in D(\mathcal{A})$ be a semi-free DG $\mathcal{A}$-module. We have $\pi\left(h^{Z}\right) \cong \pi(P)$ and the map

$$
\pi: \operatorname{Hom}_{D(\mathcal{A})}\left(h^{Y}[k], P\right) \longrightarrow \operatorname{Hom}_{D(\mathcal{A}) / L}\left(\pi\left(h^{Y}\right)[k], \pi(P)\right)
$$

is an isomorphism.

Consider the stupid truncation $\sigma_{\leq m} P$ for some $m>k$. We have a commutative diagram

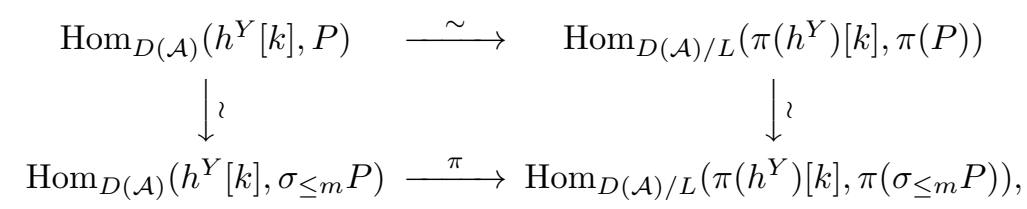


where the right vertical arrow is an isomorphism by Corollary 3.3 . Hence, the lower horizontal arrow is an isomorphism too.

On the other hand, by Lemma 5.1 and Proposition 3.4 there exist isomorphisms $\theta_{Y}: F_{1}\left(h^{Y}\right) \stackrel{\sim}{\rightarrow} F_{2}\left(h^{Y}\right)$ and $\theta_{\leq m}: F_{1}\left(\sigma_{\leq m} P\right) \stackrel{\sim}{\rightarrow} F_{2}\left(\sigma_{\leq m} P\right)$ such that the diagram

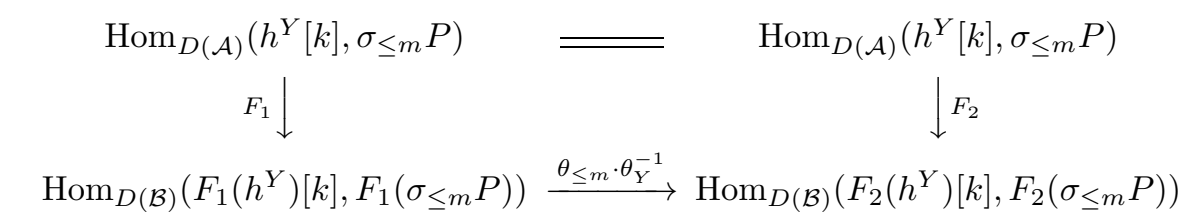

commutes. Since $F_{1} \cong H^{0}(\rho) \cdot \pi$ and $F_{2}=H^{0}(\phi) \cdot \tilde{\epsilon} \cdot \pi$ we obtain the following commutative diagram:

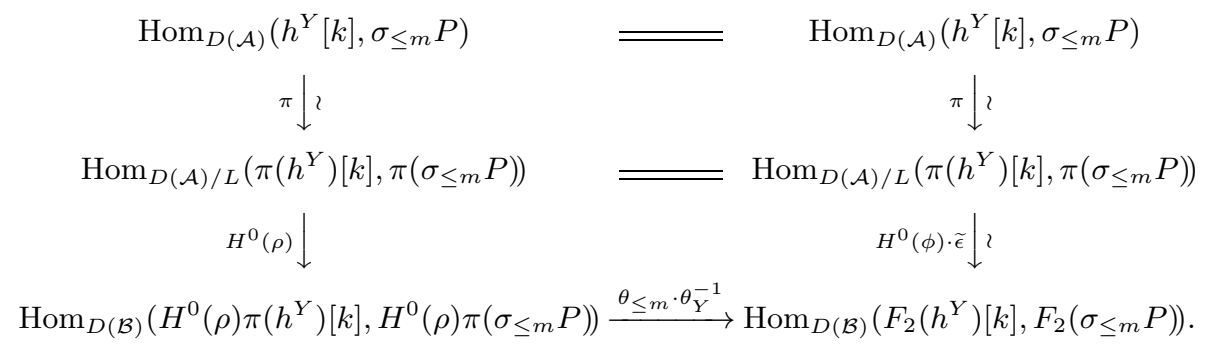

In this diagram all arrows, except possibly $H^{0}(\rho)$, are isomorphisms. Hence $H^{0}(\rho)$ is an isomorphism as well. Finally we obtain that in the commutative diagram

$$
\begin{array}{ccc}
\operatorname{Hom}_{D(\mathcal{A}) / L}\left(\pi\left(h^{Y}\right)[k], \pi(P)\right) & \stackrel{\sim}{\longrightarrow} \operatorname{Hom}_{D(\mathcal{A}) / L}\left(\pi\left(h^{Y}\right)[k], \pi\left(\sigma_{\leq m} P\right)\right) \\
H^{0}(\rho) \downarrow & H^{0}(\rho) \downarrow \\
\operatorname{Hom}_{D(\mathcal{B})}\left(H^{0}(\rho) \pi\left(h^{Y}\right)[k], H^{0}(\rho) \pi(P)\right) & \stackrel{\sim}{\longrightarrow} \operatorname{Hom}_{D(\mathcal{B})}\left(H^{0}(\rho) \pi\left(h^{Y}\right)[k], H^{0}(\rho) \pi\left(\sigma_{\leq m} P\right)\right)
\end{array}
$$

the horizontal arrows are isomorphisms by Corollary 3.3 and the right arrow is also an isomorphism as proved above. Thus we get that the canonical map

$$
H^{0}(\rho): \operatorname{Hom}\left(\pi\left(h^{Y}\right)[k], \pi\left(h^{Z}\right)\right) \longrightarrow \operatorname{Hom}\left(H^{0}(\rho) \pi\left(h^{Y}\right)[k], H^{0}(\rho) \pi\left(h^{Z}\right)\right)
$$

is an isomorphism.

Thus we proved that $H^{0}(\rho)$ is fully faithful. Since the image of $H^{0}(\rho)$ contains the set of compact generators $\mathcal{B} \subset D(\mathcal{B})$ and is closed under taking arbitrary direct sums, the functor $H^{0}(\rho)$ is essentially surjective. Therefore, it is an equivalence. This proves the lemma and the theorem.

Theorem 5.4. Let $\mathcal{A}$ be a small category which we consider as a $D G$ category and $L \subset D(\mathcal{A})$ be a localizing subcategory with the quotient functor $\pi: D(\mathcal{A}) \rightarrow D(\mathcal{A}) / L$ that has a right adjoint (Bousfield localization) $\mu$. Assume that the following conditions hold:

a) for every $Y \in \mathcal{A}$ the object $\pi\left(h^{Y}\right) \in D(\mathcal{A}) / L$ is compact;

b) for every $Y, Z \in \mathcal{A}$ we have $\operatorname{Hom}\left(\pi\left(h^{Y}\right), \pi\left(h^{Z}\right)[i]\right)=0$ when $i<0$.

Let $\mathcal{E}$ be a $D G$ category and let $F: D(\mathcal{A}) / L \rightarrow H^{0}(\mathcal{E})$ be a fully faithful functor. Then there is a quasi-functor $\mathcal{F}: \mathcal{S} \mathcal{F}(\mathcal{A}) / \mathcal{L} \cap \mathcal{S F}(\mathcal{A}) \rightarrow \mathcal{E}$ such that

(1) the functor $H^{0}(\mathcal{F}): D(\mathcal{A}) / L \rightarrow H^{0}(\mathcal{E})$ is also fully faithful;

(2) $H^{0}(\mathcal{F})(X) \cong F(X)$ for any $X \in D(\mathcal{A}) / L$. 
Proof. (1) Let $\mathcal{D} \subset \mathcal{E}$ be a full DG subcategory that consists of all objects in the essential image of $F$. Then the DG category $\mathcal{D}$ is another enhancement for $D(\mathcal{A}) / L$ and the functor $F$ induces an equivalence $\widetilde{\epsilon}:(D(\mathcal{A}) / L) \stackrel{\sim}{\rightarrow} H^{0}(\mathcal{D})$ between the triangulated categories.

As in the proof of Theorem 2.7 we denote by $\mathcal{B} \subset \mathcal{D}$ the full DG subcategory with the set of objects $\left\{\tilde{\epsilon} \pi\left(h^{Y}\right)\right\}_{Y \in \mathcal{A}}$. By Proposition 1.17, a DG functor $\Phi: \mathcal{D} \rightarrow$ $\mathcal{M}$ od $-\mathcal{B}$ induces a quasi-functor $\phi: \mathcal{D} \rightarrow \mathcal{S F}(\mathcal{B})$ such that $H^{0}(\phi): H^{0}(\mathcal{D}) \stackrel{\sim}{\longrightarrow}$ $D(\mathcal{B})$ is an equivalence.

By construction in Section 4 (formula (4.2) $)$ we have a quasi-functor

$$
\widetilde{\rho}: \mathcal{S F}(\mathcal{A}) \longrightarrow \mathcal{S F}(\mathcal{B})
$$

and by Lemma 5.2 the quasi-functor $\widetilde{\rho}$ factors through the DG quotient $\mathcal{S} \mathcal{F}(\mathcal{A}) / \mathcal{L} \cap$ $\mathcal{S F}(\mathcal{A})$. Hence it induces a quasi-functor $\rho: \mathcal{S F}(\mathcal{A}) / \mathcal{L} \cap \mathcal{S F}(\mathcal{A}) \rightarrow \mathcal{S F}(\mathcal{B})$. In Lemma 5.3 we proved that our quasi-functor $\rho$ is a quasi-equivalence. We denote by $\mathcal{F}$ the composition of quasi-functors $\rho, \phi^{-1}$ and the full embedding of $\mathcal{D}$ to $\mathcal{E}$. It is evident that $H^{0}(\mathcal{F})$ is fully faithful by construction.

(2) Lemma 5.1 implies that there is an isomorphism of functors $\theta: H^{0}(\mathcal{F}) \cdot \pi \cdot h^{\bullet} \stackrel{\sim}{\rightarrow}$ $F \cdot \pi \cdot h^{\bullet}$ from $\mathcal{A}$ to $H^{0}(\mathcal{E})$. By Proposition 3.4 applied to $\mathcal{U}=\mathcal{S F}^{-}(\mathcal{A})$ there is an isomorphism $H^{0}(\mathcal{F}) \pi(P) \cong F \pi(P)$ for any $P \in \mathcal{S} \mathcal{F}^{-}(\mathcal{A})$.

Denote as above the functor $H^{0}(\rho) \pi$ and $H^{0}(\phi) \widetilde{\epsilon} \pi$ from $\mathcal{S F}(\mathcal{A})$ to $\mathcal{S F}(\mathcal{B})$ by $F_{1}$ and $F_{2}$, respectively. Now let $P \in \mathcal{S F}(\mathcal{A})$ be any semi-free DG module. Consider its truncations $\sigma_{\leq m} P \in \mathcal{S F}^{-}(\mathcal{A})$. The object $P$ is isomorphic to a homotopy limit of the truncations $\sigma_{\leq m} P$ in $D(\mathcal{A})$; i.e., there is an exact triangle

$$
P \longrightarrow \prod_{m} \sigma_{\leq m} P \longrightarrow \prod_{m} \sigma_{\leq m} P \text {. }
$$

We have a natural map $\kappa_{P}: F_{i}(P) \rightarrow \underline{\text { holim }} F_{i}\left(\sigma_{\leq m} P\right)$. By Lemma 3.2 for any $Y \in \mathcal{A}$ and $j \in \mathbb{Z}$ we have $\operatorname{Hom}\left(F_{i}\left(h^{Y}\right), F_{i}\left(\sigma_{\geq m} P\right)[j]\right)=0$ when $m>j$. Therefore, the map

$$
\operatorname{Hom}\left(F_{i}\left(h^{Y}\right), F_{i}(P)[j]\right) \stackrel{\sim}{\longrightarrow} \operatorname{Hom}\left(F_{i}\left(h^{Y}\right), \underset{\operatorname{holim}}{\longleftarrow} F_{i}\left(\sigma_{\leq m} P\right)[j]\right)
$$

is an isomorphism for any $Y$ and $j$. Since $F_{i}\left(h^{Y}\right)$ forms a set of compact generators of $\mathcal{S F}(\mathcal{B})$ the map $\kappa_{P}$ is an isomorphism.

Now we construct an isomorphism $\vartheta_{P}: F_{1}(P) \rightarrow F_{2}(P)$. By Proposition 3.4 there are isomorphisms $\theta^{m}: F_{1}\left(P^{m}\right) \stackrel{\sim}{\rightarrow} F_{2}\left(P^{m}\right)$ for all $m$ and $\theta_{\leq 0}: F_{1}\left(\sigma_{\leq 0} P\right) \stackrel{\sim}{\rightarrow}$ $F_{2}\left(\sigma_{\leq 0} P\right)$ making the following square commutative:

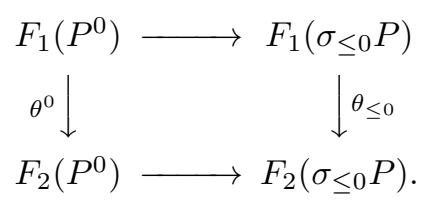

For every $m \geq 0$ we will construct an isomorphism $\vartheta_{\leq m}: F_{1}\left(\sigma_{\leq m} P\right) \stackrel{\sim}{\rightarrow}$ $F_{2}\left(\sigma_{\leq m} P\right)$ such that the diagram

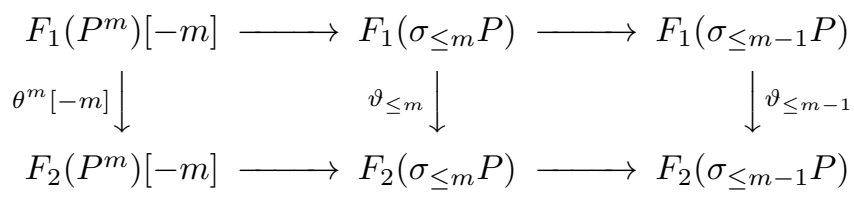


commutes for $m>0$. The proof uses the (ascending) induction on $m$ with the base of induction for $m=0$. Assume that the map $\vartheta_{\leq m}: F_{1}\left(\sigma_{\leq m} P\right) \stackrel{\sim}{\rightarrow} F_{2}\left(\sigma_{\leq m} P\right)$ exists for some $m \geq 0$. We claim that in the natural diagram

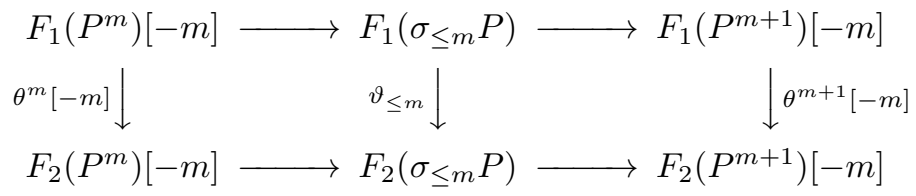

the right square commutes. Indeed, the outside square commutes by Proposition 3.4 and the left one commutes by the induction assumption. Further, we have that the map

$$
\operatorname{Hom}\left(F_{1}\left(\sigma_{\leq m} P\right), F_{1}\left(P^{m+1}\right)[-m]\right) \rightarrow \operatorname{Hom}\left(F_{1}\left(P^{m}\right)[-m], F_{1}\left(P^{m+1}\right)[-m]\right)
$$

is injective, because

$$
\begin{aligned}
\operatorname{Hom}\left(F_{1}\left(\sigma_{\leq m-1} P\right), F_{1}\left(P^{m+1}\right)[-m]\right) & =\operatorname{Hom}\left(\underline{\operatorname{hocolim}}_{k} F_{1}\left(P^{[k, m-1]}\right)[m], F_{1}\left(P^{m}\right)\right) \\
& =0
\end{aligned}
$$

by property 3 ) of $(*)$. Using the compositions with $\theta^{m+1}$ we conclude that the natural map

$$
\operatorname{Hom}\left(F_{1}\left(\sigma_{\leq m} P\right), F_{2}\left(P^{m+1}\right)[-m]\right) \rightarrow \operatorname{Hom}\left(F_{1}\left(P^{m}\right)[-m], F_{2}\left(P^{m+1}\right)[-m]\right)
$$

is also injective. Therefore, the right square in the above diagram commutes too. Hence we can find $\vartheta_{\leq m+1}$ so that the diagram

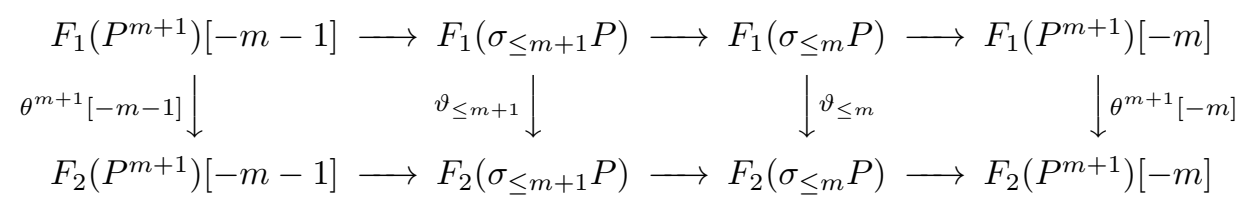

commutes. This provides the induction step. Since $F_{i}(P) \cong \underset{\text { holim }}{\longleftarrow} F_{i}\left(\sigma_{\leq m} P\right)$, there exists an isomorphism $\vartheta_{P}: F_{1}(P) \stackrel{\sim}{\rightarrow} F_{2}(P)$ that gives an isomorphism of triangles

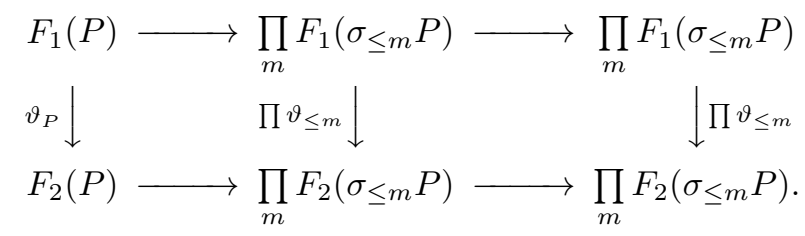

Thus, $H^{0}(\mathcal{F}) \pi(P) \cong F \pi(P)$ for any $P \in \mathcal{S F}(\mathcal{A})$.

\section{Proof of Theorem 2.8}

As in Section 4, let $\mathcal{C}$ be a pretriangulated DG category and $\epsilon:(D(\mathcal{A}) / L)^{c} \stackrel{\sim}{\rightarrow}$ $H^{0}(\mathcal{C})$ be an equivalence of triangulated categories. Denote by $\mathcal{B} \subset \mathcal{C}$ the full DG subcategory with the set of objects $\left\{\epsilon \pi\left(h^{Y}\right)\right\}_{Y \in \mathcal{A}}$. As in formula (1.1) there is the canonical DG functor $\Psi: \mathcal{C} \rightarrow \mathcal{M o d}-\mathcal{B}$ which induces a quasi-functor $\psi: \mathcal{C} \rightarrow$ $\mathcal{S F}(\mathcal{B})$ and the functor

$$
H^{0}(\psi): H^{0}(\mathcal{C}) \longrightarrow H^{0}(\mathcal{S F}(\mathcal{B})) \cong D(\mathcal{B}) .
$$

Objects $\left\{\epsilon \pi\left(h^{Y}\right)\right\}_{Y \in \mathcal{A}}$ classically generate the category $(D(\mathcal{A}) / L)^{c}$ by Theorem 1.21. Hence by Proposition 1.16 the functor $H^{0}(\psi)$ induces an equivalence between $H^{0}(\mathcal{C})$ and $\operatorname{Perf}(\mathcal{B})=D(\mathcal{B})^{c}$. 
Consider the DG category of perfect DG modules $\operatorname{Per} f(\mathcal{B})$, which by definition is the full DG subcategory of $\mathcal{S F}(\mathcal{B})$ consisting of all DG modules which are homotopy equivalent to a direct summand of a finitely generated semi-free DG module. The quasi-functor $\psi$ induces a quasi-functor $\chi: \mathcal{C} \rightarrow \mathcal{P} \operatorname{erf}(\mathcal{B})$ that is quasi-equivalence; i.e., the induced functor

$$
H^{0}(\chi): H^{0}(\mathcal{C}) \stackrel{\sim}{\longrightarrow} D(\mathcal{B})^{c}=\operatorname{Perf}(\mathcal{B})
$$

is an equivalence. Thus the DG category $\operatorname{Perf}(\mathcal{B})$ is another enhancement of $(D(\mathcal{A}) / L)^{c}$ which is equivalent to $\mathcal{C}$ via the quasi-functor $\chi$. Therefore it is sufficient to prove that $\operatorname{Perf}(\mathcal{B})$ is quasi-equivalent to a natural enhancement coming from the DG quotient. More precisely, we can consider a full DG subcategory $\mathcal{V} \subset$ $\mathcal{S F}(\mathcal{A}) / \mathcal{L} \cap \mathcal{S F}(\mathcal{A})$ which consists of all objects that belong to the subcategory of compact objects $(D(\mathcal{A}) / L)^{c}$. Thus we have to show that the DG category $\mathcal{V}$ is quasi-equivalent to $\mathcal{P} \operatorname{erf}(\mathcal{B})$.

By construction in Section 4, formulas (4.2) and (4.3) give a quasi-functor

$$
\widetilde{\rho}: \mathcal{S F}(\mathcal{A}) \longrightarrow \mathcal{S F}(\mathcal{B})
$$

and the induced functor $F_{1}=H^{0}(\widetilde{\rho}): D(\mathcal{A}) \rightarrow D(\mathcal{B})$. The restriction of $F_{1}$ to the subcategory of compact objects $D(\mathcal{A})^{c}$ induces a functor

$$
G_{1}: \operatorname{Perf}(\mathcal{A})=D(\mathcal{A})^{c} \longrightarrow D(\mathcal{B})^{c}=\operatorname{Perf}(\mathcal{B}) .
$$

Denote by $G_{2}$ the composition of $H^{0}(\chi)$ with the equivalence $\epsilon$ and the localization $\pi$ :

$$
G_{2}: \operatorname{Perf}(\mathcal{A})=D(\mathcal{A})^{c} \stackrel{\pi}{\longrightarrow}(D(A) / L)^{c} \stackrel{\epsilon}{\longrightarrow} H^{0}(\mathcal{C}) \stackrel{H^{0}(\chi)}{\longrightarrow} D(\mathcal{B})^{c}=\operatorname{Perf}(\mathcal{B}) .
$$

Thus we have two functors $G_{1}$ and $G_{2}$ from $D(\mathcal{A})^{c}$ to $D(\mathcal{B})^{c}$ and both of them enjoy the properties 1$), 2), 3)$ of $(*)$. By construction of $G_{2}$ and $a$, the composition of $h^{\bullet}: \mathcal{A} \rightarrow \mathcal{D}(\mathcal{A})^{c}$ and $G_{2}$ coincides with $\mathcal{A} \stackrel{a}{\rightarrow} H^{0}(\mathcal{B}) \rightarrow D(\mathcal{B})^{c}$. The commutativity of diagram (4.1) immediately proves the following lemma.

Lemma 6.1. There exists an isomorphism $\theta: G_{1} \cdot h^{\bullet} \stackrel{\sim}{\rightarrow} G_{2} \cdot h \bullet$ of functors from $\mathcal{A}$ to $D(\mathcal{B})^{c}$.

As in the proof of Theorem 2.7 first, we show that the following lemma holds.

Lemma 6.2. The quasi-functor $\widetilde{\rho}: \mathcal{S F}(\mathcal{A}) \rightarrow \mathcal{S F}(\mathcal{B})$ factors through the $D G$ quotient $\mathcal{S F}(\mathcal{A}) / \mathcal{L} \cap \mathcal{S F}(\mathcal{A})$.

Proof. By the Drinfeld Theorem 1.3 it is sufficient to show that the induced functor $F_{1}: D(\mathcal{A}) \rightarrow D(\mathcal{B})$ factors through the quotient $D(\mathcal{A}) / L$. Let $P \in \mathcal{P} \operatorname{erf}(\mathcal{A})$ be a perfect DG module that belongs to $\mathcal{L}$. By Lemma 6.1 and Proposition 3.4 we have

$$
G_{1}(P) \cong G_{2}(P)=H^{0}(\chi) \cdot \epsilon \cdot \pi(P)=0 .
$$

By assumption, the subcategory $L$ is generated by objects compact in $D(\mathcal{A})$. Since $F_{1}$ commutes with direct sums we get that $F_{1}(P)=0$ for any $P \in \mathcal{S F}(\mathcal{A}) \cap \mathcal{L}$. Hence, the functor $F_{1}: D(\mathcal{A}) \rightarrow D(\mathcal{B})$ factors through the quotient $D(\mathcal{A}) / L$, and, by Theorem 1.3 there is a quasi-functor

$$
\rho: \mathcal{S F}(\mathcal{A}) / \mathcal{L} \cap \mathcal{S F}(\mathcal{A}) \longrightarrow \mathcal{S F}(\mathcal{B})
$$

with an isomorphism of functors $F_{1}=H^{0}(\widetilde{\rho}) \cong H^{0}(\rho) \cdot \pi$.

Next we would like to prove the following lemma. 
Lemma 6.3. The functor $H^{0}(\rho): D(\mathcal{A}) / L \longrightarrow D(\mathcal{B})$ is an equivalence.

Proof. We already know that $H^{0}(\rho)$ is essentially surjective, because the image of $H^{0}(\rho)$ contains the set of compact generators $\mathcal{B} \subset D(\mathcal{B})$ and is closed under taking arbitrary direct sums. Now we show that it is full and faithful.

As usual, since the set $\left\{\pi\left(h^{Y}\right)\right\}_{Y \in \mathcal{A}}$ is a set of compact generators for $D(\mathcal{A}) / L$, and the functor $H^{0}(\rho)$ preserves direct sums, it suffices to prove that for any $Y, Z \in$ $\mathcal{A}$, and any $k \in \mathbb{Z}$ the map

$$
\begin{array}{r}
H^{0}(\rho): \operatorname{Hom}\left(\pi\left(h^{Y}\right)[k], \pi\left(h^{Z}\right)\right) \longrightarrow \operatorname{Hom}\left(H^{0}(\rho) \pi\left(h^{Y}\right)[k], H^{0}(\rho) \pi\left(h^{Z}\right)\right) \\
\cong \operatorname{Hom}\left(G_{1}\left(h^{Y}\right)[k], G_{1}\left(h^{Z}\right)\right)
\end{array}
$$

is an isomorphism.

By Theorem 1.21, since $L$ is generated by compact objects, the natural functor $D(\mathcal{A})^{c} / L^{c} \rightarrow(D(\mathcal{A}) / L)^{c}$ is fully faithful. Hence, by definition of localization, any morphism $f: \pi\left(h^{Y}\right)[k] \rightarrow \pi\left(h^{Z}\right)$ is represented by a pair $(g, s)$ in $D(\mathcal{A})^{c}$ of the form

$$
h^{Y}[k] \stackrel{g}{\longrightarrow} P \stackrel{s}{\longleftarrow} h^{Z},
$$

where $P$ is a perfect DG module and a cone of $s$ belongs to $L^{c}$; i.e., $\pi(s)$ is an isomorphism and $f=\pi(s)^{-1} \pi(g)$.

Full. Consider a morphism $f_{1} \in \operatorname{Hom}\left(G_{1}\left(h^{Y}\right)[k], G_{1}\left(h^{Z}\right)\right)$. The isomorphism $\theta: G_{1} \cdot h \stackrel{\sim}{\rightarrow} G_{2} \cdot h^{\bullet}$ induces a morphism $f_{2}=\theta_{Z} \cdot f_{1} \cdot \theta_{Y}[k]^{-1}$ from $G_{2}\left(h^{Y}\right)[k]$ to $G_{2}\left(h^{Z}\right)$.

The functor $G_{2}$ is the composition $H^{0}(\chi) \cdot \epsilon \cdot \pi$ and $H^{0}(\chi) \cdot \epsilon:(D(\mathcal{A}) / L)^{c} \stackrel{\sim}{\rightarrow} H^{0}(\mathcal{B})$ is an equivalence.

Denote by $f: \pi\left(h^{Y}\right)[k] \rightarrow \pi\left(h^{Z}\right)$ the morphism for which $H^{0}(\chi) \cdot \epsilon(f)=f_{2}$. By Proposition 3.4 applied to $\mathcal{U}=\operatorname{Per} f(\mathcal{A})$ there is an isomorphism $\theta_{P}: G_{1}(P) \stackrel{\sim}{\rightarrow}$ $G_{2}(P)$ such that in the following diagram

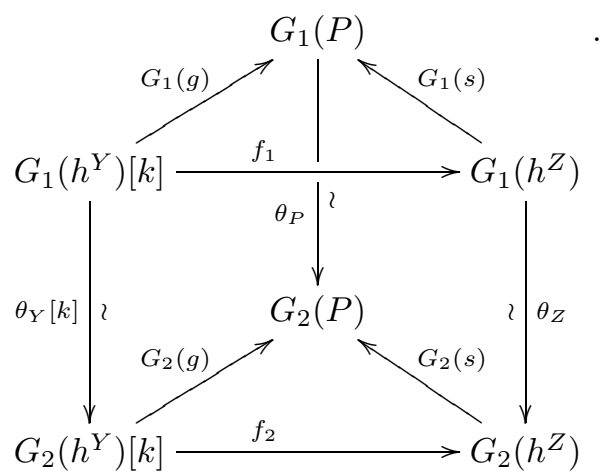

the three squares are commutative and the lower triangle is also commutative. Now the following sequence of equalities,

$$
\begin{aligned}
f_{1} & =H^{0}(\rho)\left(\pi(s)^{-1}\right) \cdot G_{1}(s) \cdot f_{1}=H^{0}(\rho)\left(\pi(s)^{-1}\right) \cdot G_{1}(s) \cdot \theta_{Z}^{-1} \cdot f_{2} \cdot \theta_{Y}[k] \\
& =H^{0}(\rho)\left(\pi(s)^{-1}\right) \cdot \theta_{P}^{-1} \cdot G_{2}(s) \cdot f_{2} \cdot \theta_{Y}[k]=H^{0}(\rho)\left(\pi(s)^{-1}\right) \cdot \theta_{P}^{-1} \cdot G_{2}(g) \cdot \theta_{Y}[k] \\
& =H^{0}(\rho)\left(\pi(s)^{-1}\right) \cdot G_{1}(g)=H^{0}(\rho)\left(\pi(s)^{-1} \pi(g)\right)=H^{0}(\rho)(f),
\end{aligned}
$$

show us that $f_{1}$ is in the image of the functor $H^{0}(\rho)$. 
Faithful. Consider a morphism $f: \pi\left(h^{Y}\right)[k] \rightarrow \pi\left(h^{Z}\right)$. As above there exist a perfect DG $\mathcal{A}$-module $P \in \mathcal{P} \operatorname{erf}(\mathcal{A})$ and a pair of morphisms $(g, s)$ in $D(\mathcal{A})^{c}$ of the form

$$
h^{Y}[k] \stackrel{g}{\longrightarrow} P \stackrel{s}{\longleftarrow} h^{Z}
$$

such that the morphism $\pi(s)$ is an isomorphism and $f=\pi(s)^{-1} \pi(g)$.

Denote by $f_{1}$ and $f_{2}$ the images of $f$ under the functors $H^{0}(\rho)$ and $H^{0}(\chi) \cdot \epsilon$, respectively. Consider again the diagram (6.1). Now both triangles are commutative and both back squares are commutative too. Assume that $f_{1}=H^{0}(\rho)(f)=0$. Then $G_{1}(g)=0$ and, as a consequence, $G_{2}(g)=0$. Thus we get

$$
f_{2}=H^{0}(\chi) \epsilon\left(\pi(s)^{-1}\right) G_{2}(s) f_{2}=H^{0}(\chi) \epsilon\left(\pi(s)^{-1}\right) G_{2}(g)=0 .
$$

But the functor $H^{0}(\chi) \epsilon$ is an equivalence. We conclude that $f=0$. This proves faithfulness.

Thus, our quasi-functor $\rho: \mathcal{S F}(\mathcal{A}) / \mathcal{L} \cap \mathcal{S F}(\mathcal{A}) \longrightarrow \mathcal{S F}(\mathcal{B})$ is a quasi-equivalence and it induces a quasi-functor between subcategories of compact objects $\rho^{c}: \mathcal{V} \rightarrow$ $\mathcal{P} \operatorname{erf}(\mathcal{B})$, where $\mathcal{V} \subset \mathcal{S F}(\mathcal{A}) / \mathcal{L} \cap \mathcal{S F}(\mathcal{A})$ is the full DG subcategory which consists of all compact objects $(D(\mathcal{A}) / L)^{c}$, and $\rho^{c}$ is a quasi-equivalence as well. This finishes the proof of Theorem 2.8

More precisely we have proved the following theorem.

Theorem 6.4. Let $\mathcal{A}$ be a small category which we consider as a DG category and $L \subset D(\mathcal{A})$ be a localizing subcategory that is generated by compact objects $L^{c}=L \cap D(\mathcal{A})^{c}$. Assume that for the quotient functor $\pi: D(\mathcal{A}) \rightarrow D(\mathcal{A}) / L$ the following condition holds:

for every $Y, Z \in \mathcal{A}$ we have $\operatorname{Hom}\left(\pi\left(h^{Y}\right), \pi\left(h^{Z}\right)[i]\right)=0$ when $i<0$.

Let $\mathcal{V} \subset \mathcal{S F}(\mathcal{A}) / \mathcal{L} \cap \mathcal{S F}(\mathcal{A})$ be the full $D G$ subcategory which consists of all compact objects $(D(\mathcal{A}) / L)^{c}$. Let $\mathcal{E}$ be another pretriangulated $D G$ category and $N:(D(\mathcal{A}) / L)^{c} \rightarrow H^{0}(\mathcal{E})$ be a fully faithful functor. Then there is a quasi-functor $\mathcal{N}: \mathcal{V} \rightarrow \mathcal{E}$ such that

(1) the functor $H^{0}(\mathcal{N}):(D(\mathcal{A}) / L)^{c} \rightarrow H^{0}(\mathcal{E})$ is also fully faithful;

(2) there is an isomorphism of functors $\theta: H^{0}(\mathcal{N}) \cdot \pi \cdot h^{\bullet} \stackrel{\sim}{\rightarrow} N \cdot \pi \cdot h \bullet$ from $\mathcal{A}$ to $H^{0}(\mathcal{E})$

(3) $H^{0}(\mathcal{N})(X) \cong N(X)$ for any $X \in(D(\mathcal{A}) / L)^{c}$.

Proof. Let $\mathcal{C} \subseteq \mathcal{E}$ be a full DG subcategory that consists of all objects in the essential image of $N$. Then the DG category $\mathcal{C}$ is another enhancement for $(D(\mathcal{A}) / L)^{c}$ and the functor $N$ induces an equivalence $\epsilon:(D(\mathcal{A}) / L)^{c} \stackrel{\sim}{\rightarrow} H^{0}(\mathcal{C})$ between the triangulated categories.

As in the proof of Theorem 2.8 we denote by $\mathcal{B} \subset \mathcal{C}$ the full DG subcategory with the set of objects $\left\{\epsilon \pi\left(h^{Y}\right)\right\}_{Y \in \mathcal{A}}$. By Proposition1.16, a DG functor $\Psi: \mathcal{C} \rightarrow \mathcal{M}$ od $-\mathcal{B}$ induces a quasi-functor $\chi: \mathcal{C} \rightarrow \mathcal{P} \operatorname{erf}(\mathcal{B})$ such that

$$
H^{0}(\chi): H^{0}(\mathcal{C}) \stackrel{\sim}{\longrightarrow} D(\mathcal{B})^{c}=\operatorname{Perf}(\mathcal{B})
$$

is an equivalence. By construction in Section 4 (formula (4.2) ) we have a quasifunctor

$$
\widetilde{\rho}: \mathcal{S F}(\mathcal{A}) \longrightarrow \mathcal{S F}(\mathcal{B})
$$

and by Lemma 6.2 the quasi-functor $\widetilde{\rho}$ factors through the DG quotient $\mathcal{S F}(\mathcal{A}) / \mathcal{L} \cap$ $\mathcal{S F}(\mathcal{A})$. Hence it induces a quasi-functor $\rho: \mathcal{S F}(\mathcal{A}) / \mathcal{L} \cap \mathcal{S F}(\mathcal{A}) \rightarrow \mathcal{S F}(\mathcal{B})$. In 
Lemma 6.3 we proved that our quasi-functor $\rho$ is a quasi-equivalence and, therefore, it induces a quasi-equivalence between DG subcategories of compact objects $\rho^{c}$ : $\mathcal{V} \rightarrow \operatorname{Perf}(\mathcal{B})$. Now we denote by $\mathcal{N}$ the composition of quasi-functors $\rho^{c}, \chi^{-1}$ and the full embedding of $\mathcal{C}$ to $\mathcal{E}$.

(1) It is evident that $H^{0}(\mathcal{N})$ is fully faithful by construction.

(2) Lemma 6.1 implies immediately that there is an isomorphisms of functors $\theta: H^{0}(\mathcal{N}) \cdot \pi \cdot h^{\bullet} \stackrel{\sim}{\rightarrow} N \cdot \pi \cdot h^{\bullet}$ from $\mathcal{A}$ to $H^{0}(\mathcal{E})$.

(3) By Proposition 3.4 applied to $\mathcal{U}=\mathcal{P} \operatorname{erf}(\mathcal{A})$, there is an isomorphism $H^{0}(\mathcal{N}) \pi(P) \cong N \pi(P)$ for any $P \in D(\mathcal{A})^{c}$. By Theorem 1.21 (4) any object $X \in(D(\mathcal{A}) / L)^{c}$ is a direct summand of an object $\pi(P)$, where $P \in \mathcal{S F}_{f g}(\mathcal{A})$. Since for any $Y, Z \in \mathcal{A}$ we have $\operatorname{Hom}\left(\pi\left(h^{Y}\right), \pi\left(h^{Z}\right)[i]\right)=0$, when $i<0$, we can deduce that $\operatorname{Hom}(\pi(P), \pi(P)[k])=0$ for sufficiently negative $k \ll 0$. Therefore, we do not have any homomorphisms from $H^{0}(\mathcal{N}) \pi(P)$ to $N \pi(P)[k]$ and from $N \pi(P)$ to $H^{0}(\mathcal{N}) \pi(P)[k]$ for $k \ll 0$, because these functors are fully faithful and $H^{0}(\mathcal{N}) \pi(P) \cong N \pi(P)$. This implies that for $X$ as a direct summand of $\pi(P)$ we also have

(6.3)

$$
\operatorname{Hom}\left(H^{0}(\mathcal{N})(X), N(X)[k]\right)=0, \quad \operatorname{Hom}\left(N(X), H^{0}(\mathcal{N})(X)[k]\right)=0 \quad \text { for } \quad k \ll 0 .
$$

Consider an object $X \oplus X[2 m+1]$ for sufficiently large $m \gg 0$. Its class in the Grothendieck group $K_{0}\left((D(\mathcal{A}) / L)^{c}\right)$ is equal to 0 . By Lemma 2.2 of Th it belongs to any full subcategory whose idempotent completion is $(D(\mathcal{A}) / L)^{c}$. Hence, $X \oplus$ $X[2 m+1] \cong \pi(Q)$ for some $Q \in \operatorname{Perf}(\mathcal{A})=D(\mathcal{A})^{c}$. We know that there is an isomorphism $H^{0}(\mathcal{N}) \pi(Q) \stackrel{\sim}{\rightarrow} N \pi(Q)$. The vanishing conditions (6.3) imply that such an isomorphism induces an isomorphism $H^{0}(\mathcal{N})(X) \stackrel{\sim}{\rightarrow} N(X)$.

\section{Applications to commutative and noncommutative Geometry}

Let $\mathcal{A}$ be a small category. As above we can consider $\mathcal{A}$ as a DG category and let $\mathcal{M}$ od $-\mathcal{A}$ be the DG category of DG $\mathcal{A}$-modules. We also can consider the abelian category of right $\mathcal{A}$-modules which we denote by $\operatorname{Mod}-\mathcal{A}$. The DG category of all complexes $C^{d g}(\operatorname{Mod}-\mathcal{A})$ is exactly the DG category $\mathcal{M} o d-\mathcal{A}$ and the derived category $D(\operatorname{Mod}-\mathcal{A})$ of the abelian category $\operatorname{Mod}-\mathcal{A}$ is exactly the derived category $D(\mathcal{A})$ introduced above.

Let $\mathcal{S} \subset \operatorname{Mod}-\mathcal{A}$ be a Serre (or dense) subcategory. By definition, this means that for any exact sequence

$$
0 \longrightarrow M^{\prime} \longrightarrow M \longrightarrow M^{\prime \prime} \longrightarrow 0,
$$

$M$ is in $\mathcal{S}$ if and only if both $M^{\prime}$ and $M^{\prime \prime}$ are in $\mathcal{S}$.

Definition 7.1. In this case we can define a quotient category $\operatorname{QMod}_{\mathcal{S}}(\mathcal{A}):=$ $\operatorname{Mod}-\mathcal{A} / \mathcal{S}$ as a category with the same objects as $\operatorname{Mod}-\mathcal{A}$ and

$$
\operatorname{Hom}_{\mathrm{QMod}_{\mathcal{S}}(\mathcal{A})}(M, N):=\underset{\operatorname{colim}}{\longrightarrow} \operatorname{Hom}_{\mathrm{Mod}-\mathcal{A}}\left(M^{\prime}, N / N^{\prime}\right),
$$

where $M^{\prime}$ and $N^{\prime}$ are submodules of $M$ and $N$ such that $M / M^{\prime}$ and $N^{\prime}$ are in $\mathcal{S}$.

We will assume that the subcategory $\mathcal{S}$ is localizing; i.e., it is a Serre category that is closed under direct sums in Mod $-\mathcal{A}$. (Hence it is closed under direct limits as well.) Denote by $\Pi$ the canonical quotient functor from $\operatorname{Mod}-\mathcal{A}$ to $\operatorname{QMod}_{\mathcal{S}}(\mathcal{A})$. It is exact, preserves direct sums and, since $\operatorname{Mod}-\mathcal{A}$ is an AB5-category with a set of generators and with enough injective objects, the quotient category $\operatorname{QMod}_{\mathcal{S}}(\mathcal{A})$ 
also has these properties. Moreover, in this case, the quotient functor $\Pi$ has a right adjoint functor $\Omega$ which is called a section functor. The functor $\Omega$ is left exact and it is full and faithful; i.e., the natural morphism $\Pi \Omega \rightarrow \operatorname{id}_{\mathrm{QMod}}$ is an isomorphism. All these facts are the standard theory of localization for abelian categories and can be found in $\mathrm{Ga}, \mathrm{Po}$.

The functor $\Pi$ is exact and it induces the functor between the derived categories which we also denote by

$$
\Pi: D(\mathcal{A})=D(\operatorname{Mod}-\mathcal{A}) \rightarrow D\left(\operatorname{QMod}_{\mathcal{S}}(\mathcal{A})\right) .
$$

Since $\Pi$ respects direct sums, by Theorem 4.1 in [N2], it has a right adjoint functor

$$
\mathbf{R} \Omega: D\left(\operatorname{QMod}_{\mathcal{S}}(\mathcal{A})\right) \rightarrow D(\operatorname{Mod}-\mathcal{A})=D(\mathcal{A}) .
$$

We are interested only in the case when $\Pi\left(h^{Y}\right)$ are compact in $D\left(\operatorname{QMod}_{\mathcal{S}}(\mathcal{A})\right)$ for all $Y \in \mathcal{A}$. This property is equivalent to the condition that $\mathbf{R} \Omega$ preserves direct sums. Indeed, in the following commutative diagram,

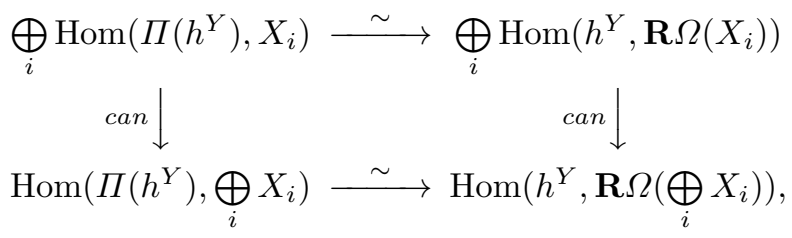

the right arrow is an isomorphism if and only if the left arrow is an isomorphism.

Now for any injective object $I \in \operatorname{QMod}_{\mathcal{S}}(\mathcal{A})$ we have $\Pi \mathbf{R} \Omega(I) \cong \Pi \Omega(I) \cong I$. Hence the functor $\mathbf{R} \Omega$ is fully faithful on the subcategory of bounded below complexes $D^{+}\left(\operatorname{QMod}_{\mathcal{S}}(\mathcal{A})\right)$. If now $\mathbf{R} \Omega$ preserves direct sums, then it is fully faithful on the whole derived category $D\left(\operatorname{QMod}_{\mathcal{S}}(\mathcal{A})\right)$.

Furthermore, for any object $M^{\cdot} \in D(\mathcal{A})$ we have an exact triangle of the form

$$
N^{\cdot} \longrightarrow M^{\cdot} \longrightarrow \mathbf{R} \Omega \Pi\left(M^{*}\right)
$$

and $\Pi\left(N^{*}\right) \cong 0$. This implies that cohomologies of $N^{\cdot}$ belong to $\mathcal{S}$. Denote by $L_{\mathcal{S}}$ the full triangulated subcategory of $D(\mathcal{A})$ that consists of all objects, cohomologies of which belong to $\mathcal{S}$. It is a localizing triangulated subcategory, because it is closed under taking direct sums. Since $\Pi\left(N^{*}\right)=0$ when $N^{\cdot} \in L_{\mathcal{S}}$, the quotient functor $\Pi: D(\mathcal{A}) \rightarrow D\left(\operatorname{QMod}_{\mathcal{S}}(\mathcal{A})\right)$ factors through the projection $\pi: D(\mathcal{A}) \rightarrow D(\mathcal{A}) / L_{\mathcal{S}}$. Moreover, it is evident now that the functor $\mathbf{R} \Omega$ in composition with $\pi$ establishes an equivalence between $D(\mathrm{QMod}(\mathcal{A}))$ and $D(\mathcal{A}) / L_{\mathcal{S}}$, because both these categories are equivalent to the right orthogonal $L_{\mathcal{S}}^{\perp}$ in $D(\mathcal{A})$. Thus we obtain

Lemma 7.2. Let $\mathcal{A}$ be a small category and $\mathcal{S} \subset \operatorname{Mod}-\mathcal{A}$ be a localizing subcategory. Let $\Pi: \operatorname{Mod}-\mathcal{A} \rightarrow \operatorname{QMod}_{\mathcal{S}}(\mathcal{A})$ be the canonical functor to the quotient category and let $L_{\mathcal{S}} \subset D(\operatorname{Mod}-\mathcal{A})$ be the full triangulated subcategory of $D(\mathcal{A})$ that consists of all objects, cohomologies of which belong to $\mathcal{S}$. Assume that the objects $\Pi\left(h^{Y}\right)$ are compact in $D\left(\mathrm{QMod}_{\mathcal{S}}(\mathcal{A})\right)$ for all $Y \in \mathcal{A}$. Then the functor $\Pi$ induces an equivalence of triangulated categories

$$
\Pi^{\prime}: D(\operatorname{Mod}-\mathcal{A}) / L_{\mathcal{S}} \stackrel{\sim}{\longrightarrow} D\left(\operatorname{QMod}_{\mathcal{S}}(\mathcal{A})\right) .
$$

Now Theorem 2.7 together with this lemma implies the following proposition.

Proposition 7.3. Let $\mathcal{A}$ be a small category and $\mathcal{S} \subset \operatorname{Mod}-\mathcal{A}$ be a localizing subcategory. Then the derived category of the quotient abelian category $D\left(\operatorname{QMod}_{\mathcal{S}}(\mathcal{A})\right)$ 
has a unique enhancement if the objects $\Pi\left(h^{Y}\right) \in D\left(\operatorname{QMod}_{\mathcal{S}}(\mathcal{A})\right)$ are compact for all $Y \in \mathcal{A}$.

As we mentioned above the quotient category $\operatorname{QMod}_{\mathcal{S}}(\mathcal{A})$ is a Grothendieck category; i.e., it is an abelian AB5-category with a set of generators. A well-known theorem of Gabriel and Popescu (see, for example, $[\mathrm{Po}$ ) states essentially that any Grothendieck category $\mathcal{C}$ is equivalent to a quotient category of the category Mod - $\Lambda$ of right modules over the endomorphism ring $\Lambda$ of a generator $U \in \mathcal{C}$. There are also Gabriel-Popescu type theorems for a set of generators.

Theorem 7.4 (Me, IENT). Let $\mathcal{C}$ be a Grothendieck category. Assume that $\mathcal{A}$ is a full subcategory of $\mathcal{C}$ such that $\mathrm{Ob} \mathcal{A}$ forms a set of generators of $\mathcal{C}$. Then there exist a localizing subcategory $\mathcal{N} \subset \operatorname{Mod}-\mathcal{A}$ and an equivalence of categories $\mathcal{C} \stackrel{\sim}{\rightarrow} \operatorname{QMod}_{\mathcal{N}}(\mathcal{A})$

Combining this theorem with Proposition 7.3 we obtain

Theorem 7.5. Let $\mathcal{C}$ be a Grothendieck category. Assume that it has a set of small generators which are compact objects in the derived category $D(\mathcal{C})$. Then the derived category $D(\mathcal{C})$ has a unique enhancement.

Now we can apply this result to the category of quasi-coherent sheaves on a quasi-compact and separated scheme. We say that a quasi-compact and separated scheme $X$ has enough locally free sheaves if for any finitely presented sheaf $\mathcal{F}$ there is an epimorphism $\mathcal{E} \rightarrow \mathcal{F}$ with a locally free sheaf $\mathcal{E}$ of finite type. In this case, the set of all locally free sheaves of finite rank forms a set of generators of the abelian category of quasi-coherent sheaves Qcoh $X$. This follows from a fact that in this case every sheaf in Qcoh $X$ is a filtering colimit of finitely presented $\mathcal{O}_{X}$-modules (see [EGA1], 6.9.12). Moreover, any locally free sheaf of finite rank is a compact object in $D(\mathrm{Qcoh} X)$, because the functor of global sections commutes with direct sums for a quasi-compact and separated scheme (see [N2, Lemma 1.4, Ex.1.10). It is also necesssary to mention that for a quasi-compact and separated scheme $X$ the category $D(\mathrm{Q} \operatorname{coh} X)$ is equivalent to the category $D(X)_{\mathrm{Qcoh}}$ of complexes of $\mathcal{O}_{X}$-modules with quasi-coherent cohomology ( $\left.\overline{\mathrm{BN}}\right]$, Cor.5.5). Thus, Theorem 7.5 implies the following corollary immediately.

Corollary 7.6. Let $X$ be a quasi-compact and separated scheme that has enough locally free sheaves. Then the derived category of quasi-coherent sheaves $D(\mathrm{Qcoh} X)$ has a unique enhancement.

Remark 7.7. The statement is also true for a quasi-compact and semi-separated scheme in the definition of [TT, B.7, because the proofs of Corollary 5.5 in $\mathrm{BN}$ and Lemma 1.4 in [N2] can be applied for a semi-separated scheme directly.

For a quasi-projective schemes by Serre's theorem we have a precise description of the category of quasi-coherent sheaves as a quotient category. Let $X$ be a quasiprojective scheme. Then it is an open subscheme of a projective scheme $\bar{X} \subset \mathbb{P}^{n}$. Denote by $A$ the following $\mathbb{Z}$-graded algebra:

$$
A=\bigoplus_{n} H^{0}\left(\bar{X}, \mathcal{O}_{\bar{X}}(n)\right) .
$$

We can consider the abelian category of all graded $A$-modules $\operatorname{Gr}(A)$. This category has a Serre subcategory of torsions modules $\operatorname{Tors}(A)$. Recall that a module $M$ is 
called torsion if for any element $x \in M$ one has $x A_{\geq p}=0$ for some $p$. Denote by $\mathrm{QGr}(A)$ the quotient category $\operatorname{Gr}(A) / \operatorname{Tors}(A)$.

With the graded algebra $A$ one can associate a $\mathbb{Z}$-category $\mathcal{A}$, objects of which are $\mathbb{Z}$ and morphisms $\operatorname{Hom}_{\mathcal{A}}(i, j)=A^{j-i}$ so that the composition in $\mathcal{A}$ comes from the multiplication in $A$. It is clear that the categories $\operatorname{Gr}(A)$, $\operatorname{Tors}(A)$, and $\operatorname{QGr}(A)$ are equivalent to $\operatorname{Mod}-\mathcal{A}$, $\operatorname{Tors}(\mathcal{A})$, and $\operatorname{QMod}(\mathcal{A})$, respectively.

The well-known Serre theorem gives us an equivalence between the category of quasi-coherent sheaves Qcoh $\bar{X}$ on $\bar{X}$ and the quotient category $\mathrm{Q} \operatorname{Gr}(A)=$ $\operatorname{QMod}(\mathcal{A})$ (see [Se, EGA2]). On the other hand, the category of quasi-coherent sheaves Qcoh $X$ on $X$ is equivalent to the quotient of the category Qcoh $\bar{X}$ by the subcategory of quasi-coherent sheaves with support on the complement $\bar{X} \backslash X$ (Ga]). Therefore, the category $\mathrm{Q} \operatorname{coh} X$ is equivalent to the quotient of the category $\operatorname{Gr}(A)=\operatorname{Mod}-\mathcal{A}$ by the localizing subcategory of $I$-torsion modules $\operatorname{Tors}_{I}(A)$, where $I$ is a homogenous ideal such that the support of the subscheme $\mathbb{P} \operatorname{roj} A / I \subset$ $\bar{X}$ is exactly $\bar{X} \backslash X$. More precisely, a graded $A$-module $M$ is called $I$-torsion if

$$
M \cong \Gamma_{I}(M)=\underline{\operatorname{colim}}_{n} \operatorname{Hom}_{A}\left(A / I^{n}, M\right) .
$$

In addition, the free modules $h^{Y}$ map to the corresponding line bundles $\left.\mathcal{O}(i)\right|_{X}$ which are compact objects in $D(\mathrm{Q} \operatorname{coh} X)$.

Corollary 7.8. The derived category of quasi-coherent sheaves $D(\mathrm{Q} \operatorname{coh} X)$ on a quasi-projective scheme $X$ has a unique enhancement.

Now let us consider the subcategory of compact objects $D(\text { Qcoh } X)^{c}$. It is well known that the subcategory of compact objects on any quasi-compact and quasiseparated scheme coincides with the subcategory of perfect complexes (see N2, $\mathrm{BvB}$ ).

Theorem 7.9. The triangulated category of perfect complexes $\operatorname{Perf}(X)$ on a quasiprojective scheme $X$ has a unique enhancement.

Proof. To apply Theorem 2.8 for the subcategory of compact objects $\operatorname{Perf}(X)=$ $D(\mathrm{Q} \operatorname{coh} X)^{c}$ we need to show that the corresponding localizing subcategory $D_{I}(\operatorname{Gr}(A))$ of all complexes, cohomologies of which are $I$-torsion modules, is compactly generated.

The functor $\Gamma_{I}: \operatorname{Gr}(A) \rightarrow \operatorname{Tors}_{I}(A)$ has a right-derived functor $\mathbf{R} \Gamma_{I}: D(\operatorname{Gr}(A))$ $\rightarrow D\left(\operatorname{Tors}_{I}(A)\right)$ via h-injective resolutions, i.e., complexes of modules $\mathcal{I}$ such that $\operatorname{Hom}(\mathcal{N}, \mathcal{I})=0$ in the homotopy category of graded $A$-modules for any acyclic complex $\mathcal{N}$ (see Sp Th.C, or [KS Ch.14 for details).

It is known that the canonical functor $i: D\left(\operatorname{Tors}_{I}(A)\right) \rightarrow D(\operatorname{Gr}(A))$ is fully faithful and realizes an equivalence of $D\left(\operatorname{Tors}_{I}(A)\right)$ with the full subcategory $D_{I}(\operatorname{Gr}(A))$. (This is proved for Noetherian rings, for example, in [Li], Cor 3.2.1.) To prove this fact it is sufficient to show that for any $C \in D_{I}(\operatorname{Gr}(A))$ the natural map $i \mathbf{R} \Gamma_{I}\left(C^{\cdot}\right) \rightarrow C^{\cdot}$ is an isomorphism. Since the functor $\mathbf{R} \Gamma_{I}$ is bounded for Noetherian schemes ([Li], Cor. 3.1.4), by the usual "way out" argument $([\mathrm{Ha}], \S 7)$ it is sufficient to check the isomorphism $i \mathbf{R} \Gamma_{I}(M) \rightarrow M$ only for $I$-torsion modules $M \in \operatorname{Tors}_{I}(A)$. But for the $I$-torsion module $M$ we have $\mathbf{R} \Gamma_{I} M \cong \Gamma_{I} M$, because $\mathbf{R} \Gamma_{I} M \cong M \otimes \mathcal{K}\left(t_{1}\right) \otimes \cdots \otimes \mathcal{K}\left(t_{m}\right)$, where $\left(t_{1}, \ldots, t_{m}\right)$ is a sequence in $A$, generating the ideal $I$ and $\mathcal{K}\left(t_{i}\right):=\left\{A \rightarrow A_{t_{i}}\right\}$ (Prop. 3.1.2 in [Li]). Now the corollary follows from the lemma 
Lemma 7.10. The subcategory $D_{I}(\operatorname{Gr}(A)) \subset D(\operatorname{Gr}(A))$ is compactly generated.

Proof. By definition it is sufficient to show that for any $C \cdot \in D_{I}(\operatorname{Gr}(A))$ there is a perfect complex $P^{\cdot}$ from $D_{I}(\operatorname{Gr}(A))$ and a nonzero morphism from $P^{\cdot}$ to $C^{\cdot}$.

Since there is an equivalence $D\left(\operatorname{Tors}_{I}(A)\right) \stackrel{\sim}{\rightarrow} D_{I}(\operatorname{Gr}(A))$ we can assume that the object $C$ is a complex of $I$-torsion modules. Consider a nontrivial cohomology of $C^{\cdot}$. Assume for simplicity that it is the 0th cohomology $H^{0}\left(C^{\cdot}\right) \neq 0$. Let us consider a nontrivial map from $A(k) \rightarrow H^{0}\left(C^{\cdot}\right)$ for some $k \in \mathbb{Z}$ and lift it to a nontrivial map $f: A(k) \rightarrow Z^{0}\left(C^{\cdot}\right)=\operatorname{Ker} d_{0}$. Thus the corresponding morphism $\tilde{f}$ : $A(k) \rightarrow Z^{0}\left(C^{\cdot}\right) \rightarrow C^{\cdot}$ is nontrivial in the derived category, and the image of $f$ is an $I$-torsion module. Let us cover the kernel Ker $f$ by a free module $T=\bigoplus_{i=1}^{p} A\left(k_{i}\right)$, and consider the Koszul complex

$$
\mathcal{K}^{\cdot}:=\left\{0 \longrightarrow \operatorname{det}(T(-k))(k) \longrightarrow \cdots \longrightarrow\left(\Lambda^{2} T(-k)\right)(k) \longrightarrow T \longrightarrow A(k) \longrightarrow 0\right\} .
$$

The cohomologies of the Koszul complex are $I$-torsion modules; hence $\mathcal{K} \cdot \in$ $D_{I}(\operatorname{Gr}(A))$. On the other hand it is perfect. Finally the map $\widetilde{f}$ can be factorized as $A(k) \rightarrow \mathcal{K}^{\cdot} \rightarrow \operatorname{Im} f \hookrightarrow Z^{0}\left(C^{\cdot}\right) \rightarrow C^{\cdot}$. Since $\tilde{f}$ is not trivial, the morphism $\mathcal{K} \cdot \rightarrow C^{\cdot}$ is also nontrivial. Therefore, the subcategory $D_{I}(\operatorname{Gr}(A))$ is compactly generated.

Thus, applying Theorem 2.8 we obtain the required statement.

\section{Bounded DERIVED CATEGORIES OF COHERENT SHEAVES}

Let as above $\mathcal{A}$ be a small category which we consider as a DG category. Let $D(\mathcal{A})$ be the derived category of DG $\mathcal{A}$-modules and $L$ be a localizing subcategory in $D(\mathcal{A})$ which is generated by objects compact in $D(\mathcal{A})$. Consider again the quotient functor $\pi: D(\mathcal{A}) \rightarrow D(\mathcal{A}) / L$. By Neeman's theorem 1.21 the objects $\pi\left(h^{Y}\right) \in$ $D(\mathcal{A}) / L$ are compact for all $Y, Z \in \mathcal{A}$. As above we assume that the following condition holds:

for every $Y, Z \in \mathcal{A}$ we have $\operatorname{Hom}\left(\pi\left(h^{Y}\right), \pi\left(h^{Z}\right)[i]\right)=0$ when $i<0$.

In this section we are going to talk about bounded derived categories of coherent sheaves. To work with these categories we introduce a notion of a triangulated subcategory of bounded and coherent objects for a general triangulated category.

Definition 8.1. Let $\mathcal{T}$ be a triangulated category that admits arbitrary direct sums. Let $S \subset \mathcal{T}^{c}$ be a set of compact generators of the category $\mathcal{T}$. We say that an object $M \in \mathcal{T}$ is compactly approximated if

a) there is $m \in \mathbb{Z}$ such that for any $Y \in S$ we have $\operatorname{Hom}(Y, M[i])=0$ when $i<m$;

b) for any $k \in \mathbb{Z}$ there is a morphism $\varphi_{k}: P_{k} \rightarrow M$ from a compact object $P_{k} \in \mathcal{T}^{c}$ such that for every $Y \in S$ the canonical map

$$
\operatorname{Hom}\left(Y, P_{k}[i]\right) \longrightarrow \operatorname{Hom}(Y, M[i])
$$

is an isomorphism when $i \geq k$.

We denote by $\mathcal{T}^{c a}$ the full subcategory of compactly approximated objects.

Remark 8.2. This definition depends on a set of compact generators $S$. In our applications this set is fixed by a construction and gives a usual bounded derived category of coherent sheaves as we see below (see Proposition 8.9). 
Remark 8.3. By Remark 1.11 the set $S$ classically generates the category of compact objects $\mathcal{T}^{c}$. Hence any compact object $P$ is a direct summand of a finite extension of finite direct sums of objects of the form $Y[i]$, where $Y \in S$. This implies that if we fix a compactly approximated object $M$ and a compact object $P$, then for any $m \in \mathbb{Z}$ there is an $s \in \mathbb{Z}$ such that for any $k \leq s$ the morphism

$$
\operatorname{Hom}\left(P, P_{k}[i]\right) \longrightarrow \operatorname{Hom}(P, M[i])
$$

induced by $\varphi_{k}: P_{k} \rightarrow M$ is an isomorphism when $i \geq m$.

This remark implies the following lemma.

Lemma 8.4. The property b) of Definition 8.1 holds if and only if $M$ is isomorphic to hocolim $P_{k}$, where $P_{0} \rightarrow P_{-1} \rightarrow \cdots$ is a sequence of morphisms of compact objects such that for every $Y \in S$ the canonical map $\operatorname{Hom}\left(Y, P_{k}[i]\right) \stackrel{\sim}{\rightarrow} \operatorname{Hom}\left(Y, P_{s}[i]\right)$ is an isomorphism when $i \geq k \geq s$.

Proof. $\Leftarrow$ If $M \cong$ hocolim $P_{k}$, then the morphisms $\varphi_{k}: P_{k} \rightarrow M$ satisfy property b) of Definition 8.1] because $\operatorname{Hom}\left(Y, P_{k}[i]\right) \cong \operatorname{Hom}(Y, M[i])$ for any $i \geq k$.

$\Rightarrow$ By Remark 8.3 for the map $\varphi_{0}: P_{0} \rightarrow M$ we can find $k_{1} \ll 0$ and a map $u_{0}: P_{0} \rightarrow P_{k_{1}}$ such that $\varphi_{k_{1}} u_{0}=\varphi_{0}$. Moreover, there are isomorphisms

$$
\operatorname{Hom}\left(Y, P_{0}[i]\right) \stackrel{\sim}{\longrightarrow} \operatorname{Hom}\left(Y, P_{k_{1}}[i]\right) \stackrel{\sim}{\longrightarrow} \operatorname{Hom}(Y, M[i])
$$

for any $i \geq 0$. Taking $\varphi_{k_{1}}: P_{k_{1}} \rightarrow M$ we can find $k_{2} \ll k_{1}$ and a map $u_{1}: P_{k_{1}} \rightarrow P_{k_{2}}$ such that $\varphi_{k_{2}} u_{1}=\varphi_{k_{1}}$. Repeating this procedure we get a sequence of morphisms $\left\{u_{i}: P_{k_{i}} \rightarrow P_{k_{i+1}}\right\}_{i \in \mathbb{N}}$ that satisfies the condition of the lemma. Now we can take $M^{\prime}=\underline{\operatorname{hocolim}} P_{k_{i}}$. The maps $\varphi_{k_{i}}$ induce a map $\varphi: M^{\prime} \rightarrow M$. It is easy to see that $\operatorname{Hom}\left(\overline{Y, M^{\prime}[i]}\right) \cong \operatorname{Hom}(Y, M[i])$ under $\varphi$ for any $i \in \mathbb{Z}$ and any $Y \in S \subset \mathcal{T}^{c}$. Since $S$ is a set of compact generators the map $\varphi$ is an isomorphism.

Lemma 8.5. In the notation as above, the compactly approximated objects $\mathcal{T}^{c a}$ form a triangulated subcategory. In addition, if all objects $Z \in S$ satisfy property a) of Definition 8.1 (with $Z=M$ ) the subcategory $\mathcal{T}^{c a}$ contains the subcategory of compact objects $\mathcal{T}^{c}$.

Proof. It is evident that any shift $M[i]$ of a compactly approximated object is compactly approximated too. It is also easy to see that a cone $C(f)$ of a map $f$ : $M \rightarrow N$ of two compactly approximated objects satisfies property a) of Definition 8.1 .

Now we need to show that the cone $C(f)$ satisfies property b) of this definition. Let us fix $k \in \mathbb{Z}$ and consider a map $\varphi_{k}: P_{k} \rightarrow M$ from Definition 8.1. Now take a sufficiently negative $m \ll k$ and a morphism $\psi_{m}: Q_{m} \rightarrow N$ from a compact object $Q_{m} \in \mathcal{T}^{c}$ as in Definition 8.1 such that the canonical map

$$
\operatorname{Hom}\left(P_{k}, Q_{m}[i]\right) \longrightarrow \operatorname{Hom}\left(P_{k}, N[i]\right)
$$

is an isomorphism when $i \geq m$. It exists by Remark 8.3 The morphism $f: M \rightarrow N$ induces a morphism from $P_{k}$ to $N$ that can be uniquely lifted to a map $f^{\prime}: P_{k} \rightarrow$ $Q_{m}$. Now morphisms $\varphi_{k}$ and $\psi_{m}$ induce a map $\chi_{k}$ from the compact object $C\left(f^{\prime}\right)$ 
to the object $C(f)$ :

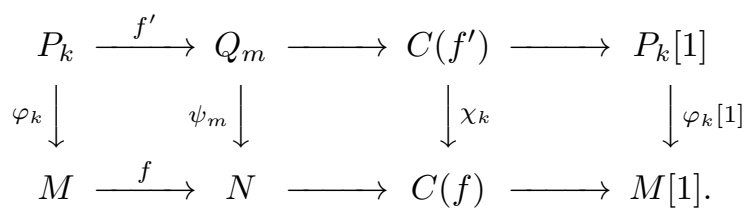

The 5-Lemma gives us isomorphisms

$$
\operatorname{Hom}\left(Y, C\left(f^{\prime}\right)[i]\right) \stackrel{\sim}{\longrightarrow} \operatorname{Hom}(Y, C(f)[i])
$$

for any $Y \in S$ and $i \geq k$. Hence, property b) of Definition 8.1 holds for the object $C(f)$ too.

If a compact object satisfies property a), then it is compactly approximated because it satisfies property b) with $\varphi_{k}$ being the identity morphism. Therefore, all objects $Y \in S$ belong to $\mathcal{T}^{c a}$. Since $S$ classically generates the subcategory of compact objects, the subcategory $\mathcal{T}^{c a}$ contains $\mathcal{T}^{c}$, because property a) obviously extends to direct summands.

Let $\mathcal{A}$ be a small category which we consider as a DG category. Let $D(\mathcal{A})$ be the derived category of DG $\mathcal{A}$-modules and $L$ be a localizing subcategory in $D(\mathcal{A})$, generated by compact objects $L^{c}=D(\mathcal{A})^{c} \cap L$. Consider the quotient functor $\pi: D(\mathcal{A}) \rightarrow D(\mathcal{A}) / L$. By Theorem 1.21 the objects $\pi\left(h^{Y}\right) \in D(\mathcal{A}) / L$ are compact for all $Y, Z \in \mathcal{A}$. We assume that the following condition holds:

for every $Y, Z \in \mathcal{A}$ we have $\operatorname{Hom}\left(\pi\left(h^{Y}\right), \pi\left(h^{Z}\right)[i]\right)=0$ when $i<0$.

The main aim of this section is to show that the triangulated subcategory of compactly approximated objects $(D(\mathcal{A}) / L)^{c a}$ with respect to the set of compact generators $\pi\left(h^{Y}\right), Y \in \mathcal{A}$ also has a unique enhancement.

Let $\mathcal{D}$ be an enhancement of this category; i.e., $\mathcal{D}$ is a pretriangulated DG category and $\bar{\epsilon}:(D(\mathcal{A}) / L)^{c a} \stackrel{\sim}{\rightarrow} H^{0}(\mathcal{D})$ is an equivalence of triangulated categories.

Denote by $\mathcal{C} \subseteq \mathcal{D}$ the full DG subcategory which consists of all objects that belong to $\bar{\epsilon}\left((D(\mathcal{A}) / L)^{c}\right)$. (By Lemma 8.5, $(D(\mathcal{A}) / L)^{c} \subseteq(D(\mathcal{A}) / L)^{c a}$.) The equivalence $\bar{\epsilon}$ induces an equivalence $\epsilon:(D(\mathcal{A}) / L)^{c} \stackrel{\sim}{\rightarrow} H^{0}(\mathcal{C})$. Thus we can apply the construction from Section 4 .

As in Section 4 denote by $\mathcal{B} \subset \mathcal{C} \subseteq \mathcal{D}$ the full DG subcategory with the set of objects $\left\{\epsilon \pi\left(h^{Y}\right)\right\}_{Y \in \mathcal{A}}$. By the construction in Section 4, formulas (4.2) and (4.3) give a quasi-functor

$$
\widetilde{\rho}: \mathcal{S F}(\mathcal{A}) \longrightarrow \mathcal{S F}(\mathcal{B})
$$

and the induced functor $F_{1}=H^{0}(\widetilde{\rho}): D(\mathcal{A}) \longrightarrow D(\mathcal{B})$.

By Lemma 6.2 the quasi-functor $\widetilde{\rho}: \mathcal{S F}(\mathcal{A}) \rightarrow \mathcal{S F}(\mathcal{B})$ can be factored through the DG quotient $\mathcal{S F}(\mathcal{A}) / \mathcal{L} \cap \mathcal{S F}(\mathcal{A})$, and we get a quasi-functor

$$
\rho: \mathcal{S F}(\mathcal{A}) / \mathcal{L} \cap \mathcal{S F}(\mathcal{A}) \longrightarrow \mathcal{S F}(\mathcal{B})
$$

such that the induced functor $H^{0}(\rho): D(\mathcal{A}) / L \longrightarrow D(\mathcal{B})$ is an equivalence by Lemma 6.3

Denote by $\mathcal{W} \subset \mathcal{S F}(\mathcal{A}) / \mathcal{L} \cap \mathcal{S F}(\mathcal{A})$ the full DG subcategory which consists of all objects that are compactly approximated in $D(\mathcal{A}) / L$, i.e., that belong to $(D(\mathcal{A}) / L)^{c a}$. Denote by $\mathcal{W}^{\prime} \subset \mathcal{S F}(\mathcal{B})$ the essential image of $\mathcal{W}$ under the quasifunctor $\rho$. It is evident that the DG subcategory $\mathcal{W}^{\prime} \subset \mathcal{S F}(\mathcal{B})$ consists of all compactly approximated objects $D(\mathcal{B})^{c a}$ (compactly approximated with respect to $\mathcal{B}$ ). 
As a consequence, we obtain a quasi-equivalence

$$
\rho^{c a}: \mathcal{W} \rightarrow \mathcal{W}^{\prime}
$$

between DG categories $\mathcal{W}$ and $\mathcal{W}^{\prime}$ that are natural enhancements of $(D(\mathcal{A}) / L)^{c a}$ and $D(\mathcal{B})^{c a}$, respectively.

Now we consider the canonical DG functor $\Phi: \mathcal{D} \rightarrow \mathcal{M o d}-\mathcal{B}$ defined by the rule

$$
\Phi(X)(B)=\operatorname{Hom}_{\mathcal{D}}(B, X), \quad \text { where } B \in \mathcal{B}, X \in \mathcal{D} .
$$

In composition with the DG quotient functor $\operatorname{Mod}-\mathcal{B} \rightarrow \mathcal{M}$ od $-\mathcal{B} / \mathcal{A} c(\mathcal{B})$ it induces a quasi-functor

$$
\phi: \mathcal{D} \longrightarrow \mathcal{S F}(\mathcal{B}) \text {. }
$$

It remains to show that $\phi$ induces a quasi-equivalence between $\mathcal{D}$ and $\mathcal{W}^{\prime}$. By Proposition 1.16, the functor $H^{0}(\phi)$ realizes an equivalence between subcategories of compact objects $H^{0}(\mathcal{C}) \cong(D(\mathcal{A}) / L)^{c}$ and $D(\mathcal{B})^{c}$. Moreover, by construction, we have isomorphisms

$$
\operatorname{Hom}_{H^{0}(\mathcal{D})}(B, X) \stackrel{\sim}{\longrightarrow} \operatorname{Hom}_{D(\mathcal{B})}\left(H^{0}(\phi) B, H^{0}(\phi) X\right)
$$

for any $B \in \mathcal{B}$ and $X \in \mathcal{D}$.

Let us denote by $Q$ the composition of functors

$$
Q:(D(\mathcal{A}) / L)^{c a} \stackrel{\bar{\epsilon}}{\longrightarrow} H^{0}(\mathcal{D}) \stackrel{H^{0}(\phi)}{\longrightarrow} D(\mathcal{B}) \stackrel{H^{0}(\rho)^{-1}}{\longrightarrow} D(\mathcal{A}) / L .
$$

Now we prove the following lemma.

Lemma 8.6. The functor $Q$ induces a functor $Q^{\prime}:(D(\mathcal{A}) / L)^{c a} \rightarrow(D(\mathcal{A}) / L)^{c a}$, which is fully faithful.

Proof. As we know, $Q\left(\pi\left(h^{Y}\right)\right) \cong \pi\left(h^{Y}\right)$ for each $Y \in \mathcal{A}$ and $Q$ gives an autoequivalence of the subcategory of compact objects $(D(\mathcal{A}) / L)^{c}$. Moreover, the isomorphism (8.2) gives an isomorphism

$$
\operatorname{Hom}\left(\pi\left(h^{Y}\right), X\right) \stackrel{\sim}{\longrightarrow} \operatorname{Hom}\left(Q\left(\pi\left(h^{Y}\right)\right), Q(X)\right)
$$

for every $Y \in \mathcal{A}$ and every $X \in(D(\mathcal{A}) / L)^{c a}$. Since the subcategory $(D(\mathcal{A}) / L)^{c}$ is the smallest triangulated subcategory which contains $\pi\left(h^{Y}\right)$ and which is closed under direct summands we have the same isomorphism

$$
\operatorname{Hom}(P, X) \stackrel{\sim}{\longrightarrow} \operatorname{Hom}(Q(P), Q(X))
$$

for any compact object $P \in(D(\mathcal{A}) / L)^{c}$. This immediately implies that any object $Q(X)$ is compactly approximated for each $X \in(D(\mathcal{A}) / L)^{c a}$. Hence, we obtain a functor

$$
Q^{\prime}:(D(\mathcal{A}) / L)^{c a} \rightarrow(D(\mathcal{A}) / L)^{c a} .
$$

Let us show that $Q$ (and consequently $Q^{\prime}$ ) is fully faithful. Let $X$ and $X^{\prime}$ be two objects of $(D(\mathcal{A}) / L)^{c a}$. Let $m$ be an integer such that $\operatorname{Hom}\left(\pi\left(h^{Y}\right), X^{\prime}[i]\right)=0$, for all $Y \in \mathcal{A}$ when $i<m$.

Fix some $k \ll m$ and consider a map $\varphi_{k}: P_{k} \rightarrow X$ as in Definition 8.1 from a compact object $P_{k}$ such that for every $\pi\left(h^{Y}\right)$ the canonical map

$$
\operatorname{Hom}\left(\pi\left(h^{Y}\right), P_{k}[i]\right) \longrightarrow \operatorname{Hom}\left(\pi\left(h^{Y}\right), X[i]\right)
$$

is an isomorphism when $i \geq k$. Denote by $C_{k}$ a cone of $\varphi_{k}$. Consider the right adjoint functor $\mu: D(\mathcal{A}) / L \rightarrow D(\mathcal{A})$ to the functor $\pi$. For any object $M \in D(\mathcal{A}) / L$ we have

$$
\operatorname{Hom}\left(h^{Y}, \mu(M)[i]\right) \cong \operatorname{Hom}\left(\pi\left(h^{Y}\right), M[i]\right) .
$$


Hence the cohomologies $H^{i}\left(\mu\left(X^{\prime}\right)\right)$ and $H^{i}\left(\mu Q\left(X^{\prime}\right)\right)$ are trivial when $i<m$.

By the same reason the cohomologies $H^{j}\left(\mu\left(C_{k}\right)\right)$ and $H^{j}\left(\mu Q\left(C_{k}\right)\right)$ are trivial when $j \geq k$. We know that the functor $\mu$ is fully faithful. Since $k \ll m$ we obtain

$$
\begin{aligned}
\operatorname{Hom}\left(C_{k}, X^{\prime}\right) & =\operatorname{Hom}\left(\mu\left(C_{k}\right), \mu\left(X^{\prime}\right)\right)=0, \\
\operatorname{Hom}\left(C_{k}[-1], X^{\prime}\right) & =\operatorname{Hom}\left(\mu\left(C_{k}\right)[-1], \mu\left(X^{\prime}\right)\right)=0 .
\end{aligned}
$$

This implies that the canonical map

$$
\operatorname{Hom}\left(X, X^{\prime}\right) \longrightarrow \operatorname{Hom}\left(P_{k}, X^{\prime}\right)
$$

is an isomorphism. For $Q\left(C_{k}\right)$ and $Q\left(X^{\prime}\right)$ there is a similar vanishing as in (8.4) and we get an isomorphism

$$
\operatorname{Hom}\left(Q(X), Q\left(X^{\prime}\right)\right) \stackrel{\sim}{\longrightarrow} \operatorname{Hom}\left(Q\left(P_{k}\right), Q\left(X^{\prime}\right)\right) .
$$

Thus we have a commutative diagram

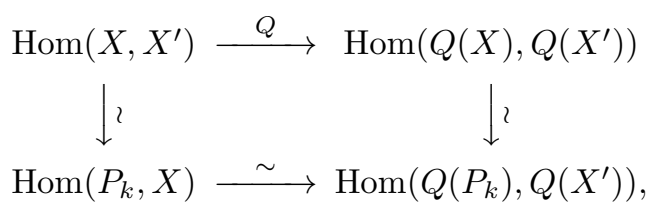

where three arrows are isomorphisms. Hence the upper arrow is also an isomorphism. This implies that the functor $Q$ is fully faithful.

Finally, we have to show that the corresponding functor $Q^{\prime}$ is essentially surjective.

Lemma 8.7. In the notation as above the functor $Q^{\prime}:(D(\mathcal{A}) / L)^{c a} \rightarrow(D(\mathcal{A}) / L)^{c a}$ is essentially surjective.

Proof. Let $X \in(D(\mathcal{A}) / L)^{c a}$ be a compactly approximated object. Suppose that it is bounded by $m \in \mathbb{Z}$ as in Definition 8.1 a) and that $X$ is isomorphic to hocolim $P_{k}$, where

$$
P_{0} \stackrel{u_{0}}{\longrightarrow} P_{-1} \stackrel{u_{1}}{\longrightarrow} \cdots \longrightarrow P_{-n} \stackrel{u_{n}}{\longrightarrow} P_{-n-1} \longrightarrow \cdots
$$

is a sequence of morphisms of compact objects such that for every $Y \in S$ the canonical map $\operatorname{Hom}\left(Y, P_{k}[i]\right) \stackrel{\sim}{\rightarrow} \operatorname{Hom}\left(Y, P_{s}[i]\right)$ is an isomorphism when $i \geq k \geq s$ as in Lemma 8.4

The functor $Q^{\prime}$ induces an autoequivalence on the subcategory of compact objects. Hence there are a sequence of compact objects $\left\{u_{i}^{\prime}: P_{-i}^{\prime} \rightarrow P_{-i-1}^{\prime}\right\}_{i \in \mathbb{N}}$ and isomorphisms $t_{i}: P_{-i} \stackrel{\sim}{\rightarrow} Q^{\prime}\left(P_{-i}^{\prime}\right)$ such that $Q^{\prime}\left(u_{i}^{\prime}\right) t_{i}=t_{i+1} u_{i}$. Consider an object $X^{\prime} \cong$ hocolim $P_{k}^{\prime}$ and denote by $\varphi_{k}^{\prime}: P_{k} \rightarrow X^{\prime}$ the respective morphisms. The object $X^{\prime}$ is bounded by the same $m \in \mathbb{Z}$ as $X$. By Lemma 8.4 it is compactly approximated. Now we have to prove that $Q^{\prime}\left(X^{\prime}\right) \cong X$.

Since $X \cong \underset{\text { hocolim }}{ } P_{k} \cong$ hocolim $Q^{\prime}\left(P_{k}^{\prime}\right)$ we have a morphism $t_{X}: X \rightarrow Q^{\prime}\left(X^{\prime}\right)$ which is induced by $Q^{\prime}\left(\varphi_{k}^{\prime}\right)$. For any $k \ll 0$ and $i \geq k$ there is a commutative diagram

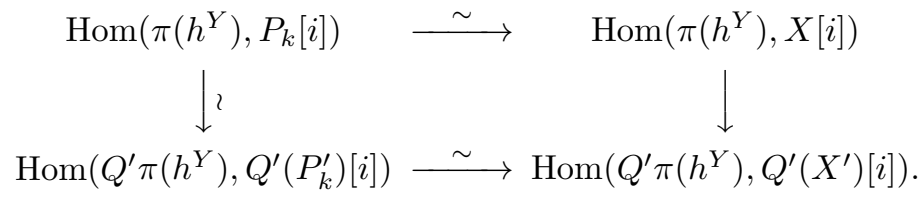


This implies that the right vertical arrow is an isomorphism for any $i$. Hence the morphism $t_{X}: X \rightarrow Q^{\prime}\left(X^{\prime}\right)$ is an isomorphism since the objects $\pi\left(h^{Y}\right)$ form a set of compact generators.

Thus we obtain the following theorem.

Theorem 8.8. Let $\mathcal{A}$ be a small category which we consider as a DG category and $L \subset D(\mathcal{A})$ be a localizing subcategory, which is generated by compact objects $L^{c}=D(\mathcal{A})^{c} \cap L$. Assume that for the quotient functor $\pi: D(\mathcal{A}) \rightarrow D(\mathcal{A}) / L$ the following condition holds:

$$
\text { for every } Y, Z \in \mathcal{A} \text { we have } \operatorname{Hom}\left(\pi\left(h^{Y}\right), \pi\left(h^{Z}\right)[i]\right)=0 \text { when } i<0 \text {. }
$$

Then the category of compactly approximated objects $(D(\mathcal{A}) / L)^{\text {ca }}$ has a unique enhancement.

Proof. Since the image of the functor $Q$ belongs to the subcategory of compactly approximated objects the quasi-functor $\phi: \mathcal{D} \rightarrow \mathcal{S F}(\mathcal{B})$ induces a quasi-functor $\phi^{c a}: \mathcal{D} \longrightarrow \mathcal{W}^{\prime}$, where $\mathcal{W}^{\prime} \subset \mathcal{S F}(\mathcal{B})$ as above is the DG subcategory which consists of all compactly approximated objects $D(\mathcal{B})^{c a}$. By Lemmas 8.6 and 8.7 the functor $Q^{\prime}$ is an equivalence. Hence the quasi-functor $\phi^{c a}$ is a quasi-equivalence as well. On the other hand, we showed above that there is a quasi-equivalence $\rho^{c a}: \mathcal{W} \rightarrow \mathcal{W}^{\prime}$, where $\mathcal{W} \subset \mathcal{S F}(\mathcal{A}) / \mathcal{L} \cap \mathcal{S F}(\mathcal{A})$ is the full DG subcategory, which consists of all compactly approximated objects in $D(\mathcal{A}) / L$. The composition

$$
\left(\phi^{c a}\right)^{-1} \cdot \rho^{c a}: \mathcal{W} \rightarrow \mathcal{D}
$$

gives a quasi-equivalence between these two different enhancements of $(D(\mathcal{A}) / L)^{c a}$.

Let $X$ be a Noetherian scheme. As above we say that $X$ has enough locally free sheaves if for any coherent sheaf $\mathcal{F}$ there is a locally free sheaf of finite type $\mathcal{E}$ and an epimorphism $\mathcal{E} \rightarrow \mathcal{F}$. For example, any quasi-projective scheme satisfies these conditions.

Proposition 8.9. Let $X$ be a Noetherian scheme that has enough locally free sheaves. Let $S=\left\{\mathcal{E}_{i}\right\}_{i \in I}$ be a set of locally free sheaves of finite type such that for any coherent sheaf $\mathcal{F}$ there is an epimorphism from a finite direct sum $\bigoplus_{j=1}^{n} \mathcal{E}_{i_{j}}$ to $\mathcal{F}$. Then the set $S$ is a set of compact generators of $D(\mathrm{Qcoh} X)$ and an object $M \in D(\mathrm{Qcoh} X)$ is compactly approximated (with respect to $S$ ) if and only if it is a cohomologically bounded complex with coherent cohomologies. Thus the triangulated subcategory of compactly approximated objects $D(\mathrm{Qcoh} X)^{\text {ca }}$ is equivalent to the bounded category of coherent sheaves $D^{b}(\operatorname{coh} X) \cong D^{b}(\mathrm{Q} \operatorname{coh} X)_{\operatorname{coh}}$.

First of all, it is known that for any Noetherian scheme $X$ the natural functor from the bounded derived category of coherent sheaves $D^{b}(\operatorname{coh} X)$ to $D(\mathrm{Q} \operatorname{coh} X)$ is fully faithful and establishes an equivalence of $D^{b}(\operatorname{coh} X)$ with the subcategory $D^{b}(\mathrm{Qcoh} X)_{\text {coh }}$ of cohomologically bounded complexes with coherent cohomologies. To prove Proposition 8.9 we will need the following lemmas.

Lemma 8.10 ([TT], B.11, B.8). Let $X$ be a Noetherian scheme. Then there is an integer $N$ such that for all $k \geq N$ and all quasi-coherent sheaves $\mathcal{F}$ we have $\operatorname{Ext}^{k}(\mathcal{E}, \mathcal{F})=0$, where $\mathcal{E}$ is a locally free sheaf of finite type. 
Lemma 8.11 ([TT], 2.3.1 e), 2.2.8). Let $X$ be a scheme as in Proposition 8.9, Then for any $M \in D^{b}(\mathrm{Q} c \mathrm{~h} X)_{\mathrm{coh}}$ there is a bounded above complex of locally free sheaves of finite type $P^{\cdot}$ with a quasi-isomorphism $P^{\cdot} \stackrel{\sim}{\rightarrow} M$.

Lemma 8.12. Let a scheme $X$ and a set of locally free sheaves of finite type $S=\left\{\mathcal{E}_{i}\right\}_{i \in I}$ be as in Proposition 8.9, Let $M \in D(\mathrm{Q} \operatorname{coh} X)$ be a complex of quasicoherent sheaves and $H^{j}(M) \neq 0$ for some $j \in \mathbb{Z}$. Then there is a sheaf $\mathcal{E}_{i} \in S$ such that $\operatorname{Hom}\left(\mathcal{E}_{i}, M[j]\right) \neq 0$.

Proof. Let us consider the stupid truncation $\sigma_{\geq j} M$. We have an epimorphism $H^{j}\left(\sigma_{\geq j} M\right) \rightarrow H^{j}(M)$. Any quasi-coherent sheaf on a Noetherian scheme is a direct limit of its coherent subsheaves. Therefore we can find a coherent subsheaf $\mathcal{F} \subset H^{j}\left(\sigma_{\geq j} M\right)$ such that the compositon map to $H^{j}(M)$ is nontrivial. By assumption any coherent sheaf can be covered by a direct sum of $\mathcal{E}_{i} \in S$. Hence, we can find a morphism $\mathcal{E}_{i} \rightarrow H^{j}\left(\sigma_{\geq j} M\right)$ such that the composition with the map to $H^{j}(M)$ is nontrivial. This map induces a map

$$
\mathcal{E}_{i} \rightarrow H^{j}\left(\sigma_{\geq j} M\right) \rightarrow \sigma_{\geq j} M[j] \rightarrow M[j],
$$

which is nontrivial, because it is nontrivial on the cohomologies.

Proof of Proposition 8.9. $\Leftarrow$ Any (cohomologically) bounded complex satisfies property a) of Definition 8.1. By Lemma 8.11 for any $M \in D^{b}(\mathrm{Q} \operatorname{coh} X)_{\operatorname{coh}}$ there is a bounded above resolution of locally free sheaves of finite type $P \stackrel{\sim}{\rightarrow} M$. To construct an approximation $\varphi_{k}: P_{k} \rightarrow M$ we can consider a stupid truncation $\sigma_{\geq l} P^{\cdot}$ for $l \ll(k-N)$, where $N$ is an integer from Lemma 8.10. The object $\sigma_{\geq l} P$. is a perfect complex and the canonical map $\varphi_{k}: \sigma_{\geq l} P^{\cdot} \rightarrow M$ satisfies the property b). Indeed, the cone $C_{k}$ of this map $\varphi_{k}$ is a cohomologically bounded complex such that $H^{j}\left(C_{k}\right)$ are trivial when $j>l$. Lemma 8.10 implies that $\operatorname{Hom}\left(\mathcal{E}_{i}, C_{k}[m]\right)=0$ for all $\mathcal{E}_{i} \in S$ when $m \geq k$. Therefore, any bounded complex of coherent sheaves is compactly approximated.

$\Rightarrow$ Let $M \in D($ Qcoh $X)$ be a compactly approximated object. By Lemma 8.12 , property a) implies that $M$ is cohomologically bounded below. On the other hand, property b) gives us that $M$ is cohomologically bounded above. Indeed, by Lemma 8.12, a cone of a map $\varphi_{k}: P_{k} \rightarrow M$ is bounded above and the object $P_{k}$ is cohomologically bounded as a perfect complex. Moreover, Lemma 8.12 implies that all cohomologies $H^{m}\left(C_{k}\right)$ of the cone $C_{k}$ of the map $\varphi_{k}$ are trivial when $m>k$. Hence, for sufficiently negative $k$, we have that nontrivial cohomologies of $M$ are isomorphic to the cohomologies of $P_{k}$. Therefore, they are coherent sheaves and $M$ is a cohomologically bounded complex with coherent cohomologies.

Theorem 8.13. The bounded derived category of coherent sheaves $D^{b}(\operatorname{coh} X)$ on a quasi-projective scheme $X$ has a unique enhancement.

Proof. Let $X$ be a quasi-projective scheme. Then it is an open subscheme of a projective scheme $\bar{X} \subset \mathbb{P}^{n}$. Denote by $A$ the following $\mathbb{Z}$-graded algebra:

$$
A=\bigoplus_{n} H^{0}\left(\bar{X}, \mathcal{O}_{\bar{X}}(n)\right) .
$$

With the graded algebra $A$ one can associate a $\mathbb{Z}$-category $\mathcal{A}$, objects of which are $\mathbb{Z}$ and $\operatorname{Hom}_{\mathcal{A}}(i, j)=A^{j-i}$ so that the composition in $\mathcal{A}$ comes from the multiplication in $A$. 
It was shown in the previous Section 7 that the category Qcoh $X$ is equivalent to a quotient of the category $\operatorname{Gr}(A)=\operatorname{Mod}-\mathcal{A}$ by the localizing subcategory of $I$-torsion sheaves $\operatorname{Tors}_{I}(\mathcal{A})$, where $I$ is a homogenous ideal such that the support of the subscheme $\mathbb{P} \operatorname{roj} A / I \subset \bar{X}$ is exactly $\bar{X} \backslash X$. Moreover, by Lemma 7.2, we also know that the derived category $D(\mathrm{Q} \operatorname{coh} X)$ is equivalent to the quotient of $D(\mathcal{A})$ by the localizing subcategoy $D_{I}(\mathcal{A}) \subset D(\mathcal{A})$ that consists of all objects, cohomologies of which belong to $\operatorname{Tors}_{I}(\mathcal{A})$. In addition, the free modules $h^{Y}$ map to the corresponding line bundles $\left.\mathcal{O}(i)\right|_{X}$ which are compact objects in $D(\mathrm{Qcoh} X)$.

By Lemma 7.10 the subcategory $D_{I}(\mathcal{A})$ is compactly generated. Hence, Theorem 8.8 implies that the bounded derived category of coherent sheaves $D^{b}(\operatorname{coh} X)$, which is equivalent to the subcategory of compactly approximated objects in $D(\mathrm{Qcoh} X)$, has a unique enhancement.

\section{Strong Uniqueness and Fully faithful FunCtors}

In this section we prove a strong uniqueness for bounded derived categories of coherent sheaves and categories of perfect complexes on projective schemes. We recall the notion of an ample sequence in an abelian category introduced in [01.

Definition 9.1. Let $\mathfrak{A}$ be a $k$-linear abelian category. Let $\left\{P_{i}\right\}_{i \in \mathbb{Z}}$ be a sequence of objects of $\mathfrak{A}$. We say that this sequence is ample if for every object $C \in \mathfrak{A}$ there exists $N$ such that for all $i<N$ the following conditions hold:

a) there is an epimorphism $P_{i}^{\oplus n_{i}} \rightarrow C$ for some $n_{i} \in \mathbb{N}$;

b) $\operatorname{Ext}^{j}\left(P_{i}, C\right)=0$ for any $j \neq 0$;

c) $\operatorname{Hom}\left(C, P_{i}\right)=0$.

Proposition 9.2. Let $X \subseteq \mathbb{P}^{N}$ be a projective scheme such that the maximal torsion subsheaf $T_{0}\left(\mathcal{O}_{X}\right) \subset \mathcal{O}_{X}$ of dimension 0 is trivial. Then the sequence $\left\{\mathcal{O}_{X}(i)\right\}_{i \in \mathbb{Z}}$ is ample in the abelian category of coherent sheaves coh $X$.

Proof. It is a classical result of Serre $\mathrm{Se}$ that for any coherent sheaf $\mathcal{G}$ on a projective scheme $X$ and for sufficiently large $m \gg 0$ the sheaf $\mathcal{G}(m)$ is generated by a finite number of global sections and $H^{j}(X, \mathcal{G}(m))=0$ for $j>0$. This implies a) and b) of Definition 9.1.

Since any coherent sheaf $\mathcal{G}$ on $X$ is covered by a $\mathcal{O}(k)^{\oplus n_{k}}$ for some $k \in \mathbb{Z}$, it is sufficient to show that $H^{0}\left(X, \mathcal{O}_{X}(m)\right)=$ for $m \ll 0$. But for $m \ll 0$,

$$
H^{0}\left(X, \mathcal{O}_{X}(m)\right)=H^{0}\left(\mathbb{P}^{N}, \mathcal{O}_{X}(m)\right)=H^{0}\left(\mathbb{P}^{N}, \mathcal{E} x t_{\mathbb{P}^{N}}^{N}\left(\mathcal{O}_{X}, \omega_{\mathbb{P}^{N}}\right)(-m)\right)=0,
$$

because by local duality the sheaf $\mathcal{E} x t_{\mathbb{P}^{N}}^{N}\left(\mathcal{O}_{X}, \omega_{\mathbb{P}^{N}}\right)$ is trivial when $T_{0}\left(\mathcal{O}_{X}\right)$ is trivial.

Let $\mathfrak{A}$ be an abelian category with an ample sequence $\left\{P_{i}\right\}$. Denote by $D^{b}(\mathfrak{A})$ the bounded derived category of $\mathfrak{A}$. Let us consider the full subcategory $j: \mathcal{P} \hookrightarrow D^{b}(\mathfrak{A})$ such that $\mathrm{Ob} \mathcal{P}:=\left\{P_{i} \mid i \in \mathbb{Z}\right\}$. The following proposition is proved in $\mathrm{O} 1, \mathrm{O} 2$.

Proposition 9.3. Let $F: D^{b}(\mathfrak{A}) \stackrel{\sim}{\rightarrow} D^{b}(\mathfrak{A})$ be an autoequivalence. Suppose there exists an isomorphism of functors $\theta_{\mathcal{P}}: j \stackrel{\sim}{\rightarrow} F \cdot j$, where $j$ is the natural embedding of $\mathcal{P}:=\left\{P_{i} \mid i \in \mathbb{Z}\right\}$ to $D^{b}(\mathfrak{A})$. Then it can be extended to an isomorphism id $\stackrel{\sim}{\rightarrow} F$ on the whole $D^{b}(\mathfrak{A})$.

We can extend this proposition to the case of exact categories. Let $\mathfrak{E}$ be an exact category. Assume that it is a full exact subcategory of an abelian category $\mathfrak{A}$, i.e., 
that $\mathfrak{E} \subset \mathfrak{A}$ is closed under extensions in $\mathfrak{A}$. We also assume that an additional property holds:

(EPI) a map $f$ in $\mathfrak{E}$ is an admissible epimorphism if and only if it is an epimorphism in $\mathfrak{A}$.

Now we define an ample sequence in $\mathfrak{E}$.

Definition 9.4. Let $\mathfrak{E}$ be a $k$-linear exact category. Let $\left\{P_{i}\right\}_{i \in \mathbb{Z}}$ be a sequence of objects of $\mathfrak{E}$. We say that the sequence $\left\{P_{i}\right\}_{i \in \mathbb{Z}}$ is ample in $\mathfrak{E}$ if there is an exact embedding $\mathfrak{E} \subset \mathfrak{A}$ in an abelian category $\mathfrak{A}$ such that the condition (EPI) holds and $\left\{P_{i}\right\}_{i \in \mathbb{Z}}$ is ample in $\mathfrak{A}$.

Starting with an exact category $\mathfrak{E}$ we can construct a derived category $D^{*}(\mathfrak{E})$. A complex $E^{\cdot}$ is called acyclic if for any $n$ the differential $d_{n}: E_{n} \rightarrow E_{n+1}$ factors as $E_{n} \stackrel{p_{n}}{\rightarrow} Z_{n} \stackrel{i_{n}}{\rightarrow} E_{n+1}$, where $p_{n}$ is a cokernel for $d_{n-1}$ and an admissible epimorphism and $i_{n}$ is a kernel for $d_{n+1}$ and an admissible monomorphism. We define a derived category $D^{*}(\mathfrak{E})$ as a quotient of the homotopy category $H^{*}(\mathfrak{E})$ by the triangulated subcategory of acyclic complexes.

Remark 9.5. If $\mathfrak{E} \subset \mathfrak{A}$ is an exact subcategory of an abelian category $\mathfrak{A}$ such that the condition (EPI) holds and for any $C \in \mathfrak{A}$ there is an epimorphism $E \rightarrow C$ from $E \in \mathfrak{E}$, then the canonical functor $D^{-}(\mathfrak{E}) \rightarrow D^{-}(\mathfrak{A})$ is an equivalence and hence the functor $D^{b}(\mathfrak{E}) \rightarrow D^{b}(\mathfrak{A})$ is fully faithful ([K2]). This follows from the fact that for any bounded above complex $C$ over $\mathfrak{A}$ with cohomologies from $\mathfrak{E}$ there is a quasi-isomorphism $E^{\cdot} \rightarrow C$, where $E^{\cdot}$ is a bounded above complex over $\mathfrak{E}$.

Proposition 9.3 can be generalized and extended to the case of exact categories.

Proposition 9.6. Let $\mathfrak{E}$ be an exact category possessing an ample sequence $\left\{P_{i}\right\}$. Let $F: D^{b}(\mathfrak{E}) \stackrel{\sim}{\rightarrow} D^{b}(\mathfrak{E})$ be an autoequivalence. Suppose there is an isomorphism of functors $\theta_{\mathcal{P}}: j \stackrel{\sim}{\rightarrow} F \cdot j$, where $\mathrm{Ob} \mathcal{P}:=\left\{P_{i} \mid i \in \mathbb{Z}\right\}$ and $j: \mathcal{P} \hookrightarrow D^{b}(\mathfrak{E})$ is a natural full embedding. Then it can be extended to an isomorphism id $\stackrel{\sim}{\rightarrow} F$ on the whole $D^{b}(\mathfrak{E})$.

Proof. A proof of this proposition is essentially the same as the proof of Proposition 9.3 and it is given in Appendix B.

Remark 9.7. Our main example of an exact category is the category of locally free sheaves of finite type $\operatorname{Loc} X$ on a projective scheme $X$. In this case the exact embedding Loc $X \subseteq \operatorname{coh} X$ satisfies condition (EPI) and the bounded derived category $D^{b}(\operatorname{Loc} X)$ is equivalent to the category of perfect complexes Perf $X \subseteq D^{b}(\operatorname{coh} X)$. If the maximal torsion subsheaf $T_{0}\left(\mathcal{O}_{X}\right) \subset \mathcal{O}_{X}$ of dimension 0 is trivial, then the sequence $\left\{\mathcal{O}_{X}(i)\right\}_{i \in \mathbb{Z}}$ is an ample sequence in Loc $X$ according to Definition 9.4

Theorem 9.8. Let $\mathfrak{E}$ be an exact category with an ample sequence $\left\{P_{i}\right\}_{i \in \mathbb{Z}}$ and $j: \mathcal{P} \hookrightarrow D^{b}(\mathfrak{E})$ be a full subcategory with $\mathrm{Ob} \mathcal{P}:=\left\{P_{i} \mid i \in \mathbb{Z}\right\}$. Assume that there is an equivalence $u: D^{b}(\mathfrak{E}) \stackrel{\sim}{\rightarrow}(D(\mathcal{P}) / L)^{c}$ (or with $\left.(D(\mathcal{P}) / L)^{c a}\right)$, where $L \subset D(\mathcal{P})$ is a localizing subcategory that is generated by compact objects $L^{c}=L \cap D(\mathcal{P})^{c}$, such that $u \cdot j \cong \pi \cdot h \bullet$ on $\mathcal{P}$. Then the category $D^{b}(\mathfrak{E})$ has a strongly unique enhancement.

Proof. (1) Let $\mathcal{E}$ be a pretriangulated DG category and $N:(D(\mathcal{P}) / L)^{c} \stackrel{\sim}{\rightarrow} H^{0}(\mathcal{E})$ be an equivalence. By Theorem 6.4 there is a quasi-equivalence $\mathcal{N}: \mathcal{V} \rightarrow \mathcal{E}$, where $\mathcal{V} \subset \mathcal{S F}(\mathcal{P}) / \mathcal{L} \cap \mathcal{S F}(\mathcal{P})$ is the full DG subcategory which consists of all compact 
objects $(D(\mathcal{P}) / L)^{c}$. Moreover, we know that there is an isomorphism of functors $\theta: H^{0}(\mathcal{N}) \cdot \pi \cdot h \stackrel{\sim}{\rightarrow} N \cdot \pi \cdot h \bullet$ from $\mathcal{P}$ to $H^{0}(\mathcal{E})$.

Consider the composition $F=u^{-1} H^{0}(\mathcal{N})^{-1} N u$ from $D^{b}(\mathfrak{E})$ to itself. There is an isomorphism $j \stackrel{\sim}{\rightarrow} F \cdot j$ on the subcategory $\mathcal{P}$. Hence we can apply Proposition 9.6 and obtain that the functor $F$ is isomorphic to the identity functor on the whole $D^{b}(\mathfrak{E})$. Therefore, the functors $H^{0}(\mathcal{N})$ and $N$ are isomorphic. Thus, any equivalence $N:(D(\mathcal{P}) / L)^{c} \stackrel{\sim}{\rightarrow} H^{0}(\mathcal{E})$ can be lifted to a quasi-equivalence $\mathcal{N}$, and the category $D^{b}(\mathfrak{E}) \cong(D(\mathcal{P}) / L)^{c}$ has strongly unique enhancement.

(2) Denote by $\mathcal{W} \subset \mathcal{S F}(\mathcal{P}) / \mathcal{L} \cap \mathcal{S F}(\mathcal{P})$ the full DG subcategory which consists of all objects that are compactly approximated in $D(\mathcal{P}) / L$, i.e., that belong to $(D(\mathcal{P}) / L)^{c a}$.

Let $\mathcal{D}$ be a pretriangulated DG category and $\bar{\epsilon}:(D(\mathcal{P}) / L)^{c a} \stackrel{\sim}{\rightarrow} H^{0}(\mathcal{D})$ be an equivalence. In the proof of Theorem 8.8 we constructed a quasi-equivalence $\left(\phi^{c a}\right)^{-1} \cdot \rho^{c a}: \mathcal{W} \rightarrow \mathcal{D}$. Moreover, by construction, the functors $H^{0}\left(\left(\phi^{c a}\right)^{-1} \cdot \rho^{c a}\right) \pi h^{\bullet}$ and $\bar{\epsilon} \pi h^{\bullet}$ from $\mathcal{P}$ to $H^{0}(\mathcal{D})$ are isomorphic. Thus, for the composition $F=$ $u^{-1} H^{0}\left(\left(\rho^{c a}\right)^{-1} \cdot \phi^{c a}\right) \cdot \bar{\epsilon} u$ we have an isomorphism $j \stackrel{\sim}{\rightarrow} F \cdot j$ on the subcategory $\mathcal{P}$. Applying Proposition 9.6 we obtain that the functor $F$ is isomorphic to the identity functor on the whole $D^{b}(\mathfrak{E})$. Therefore, the functors $H^{0}\left(\left(\phi^{c a}\right)^{-1} \cdot \rho^{c a}\right)$ and $\bar{\epsilon}$ are isomorphic. Thus any equivalence $\bar{\epsilon}:(D(\mathcal{P}) / L)^{c a} \stackrel{\sim}{\rightarrow} H^{0}(\mathcal{D})$ can be lifted to a quasi-equivalence.

This theorem immediately implies the following corollary.

Theorem 9.9. Let $X$ be a projective scheme over $k$ such that the maximal torsion subsheaf $T_{0}\left(\mathcal{O}_{X}\right) \subset \mathcal{O}_{X}$ of dimension 0 is trivial. Then the triangulated categories $\operatorname{Perf}(X)$ and $D^{b}(\operatorname{coh} X)$ have strongly unique enhancements.

Proof. Let $X$ be a projective scheme. Denote by $A$ the graded algebra $A=$ $\bigoplus_{n} H^{0}\left(X, \mathcal{O}_{X}(n)\right)$. To the graded algebra $A$ we can attach a $\mathbb{Z}$-category $\mathcal{A}$, objects of which are $\mathbb{Z}$ and $\operatorname{Hom}_{\mathcal{A}}(i, j)=A^{j-i}$ with a natural composition law.

It was explained in Section 7 that the category Qcoh $X$ is equivalent to a quotient of the category $\operatorname{Mod}-\mathcal{A}=\operatorname{Gr}(A)$ by the localizing subcategory of torsion sheaves $\operatorname{Tors}(\mathcal{A})$. Moreover, by Lemma 7.2, we also know that the derived category $D(\mathrm{Q} \operatorname{coh} X)$ is equivalent to the quotient $D(\mathcal{A}) / D_{\text {tors }}(\mathcal{A})$, where $D_{\text {tors }}(\mathcal{A}) \subset D(\mathcal{A})$ is a localizing subcategory that consists of all objects, cohomologies of which belong to Tors $(\mathcal{A})$. In addition, the free modules $h^{Y}$ map to the corresponding line bundles $\mathcal{O}_{X}(i)$ which are compact objects in $D($ Qcoh $X)$. Lemma 7.10 gives us that the subcategory $D_{\text {tors }}(\mathcal{A})$ is compactly generated. The category $\operatorname{Perf}(X)=D^{b}(\operatorname{Loc} X)$ (Remark 9.7) is equivalent to the category $\left(D(\mathcal{A}) / D_{\text {tors }}(\mathcal{A})\right)^{c}$ and by Proposition 8.9 the bounded derived category of coherent sheaves $D^{b}(\operatorname{coh} X)$ is equivalent to the triangulated category of compactly approximated objects $\left(D(\mathcal{A}) / D_{\text {tors }}(\mathcal{A})\right)^{c a} \cong$ $D(\mathrm{Q} \operatorname{coh} X)^{c a}$. These equivalences are isomorphic to the identity on the full subcategory $\mathcal{P}:=\left\{\mathcal{O}_{X}(i)\right\}_{i \in \mathbb{Z}}$. By Remark 9.7 (resp., Prop. 9.2) the sequence $\mathcal{P}:=$ $\left\{\mathcal{O}_{X}(i)\right\}_{i \in \mathbb{Z}}$ is ample in Loc $X$ (resp., $\left.\operatorname{coh} X\right)$ on a projective scheme with $T_{0}\left(\mathcal{O}_{X}\right)=$ 0 . Hence we can apply Theorem 9.8 and obtain that $\operatorname{Perf}(X)$ (resp., $D^{b}(\operatorname{coh} X)$ ) has strongly unique enhancement.

Let $X$ be a quasi-compact and quasi-separated scheme. Define a DG enhancement $\mathcal{D}_{d g}(\mathrm{Qcoh} X)$ of the derived category $D(\mathrm{Q} \operatorname{coh} X)$ as the quotient $\mathcal{C}_{d g}(\mathrm{Qcoh} X) /$ $\mathcal{A} c_{d g}(\mathrm{Q} \operatorname{coh} X)$, where $\mathcal{C}_{d g}(\mathrm{Q} \operatorname{coh} X)$ is the DG category of unbounded complexes 
over Qcoh $X$ and $\mathcal{A} c_{d g}(\mathrm{Qcoh} X)$ is the DG subcategory of unbounded acyclic complexes. Thus the DG category $\mathcal{D}_{d g}(\mathrm{Qcoh} X)$ is an enhancement of the derived category $D(Q \operatorname{coh} X)$. There is a theorem of Bertrand Toën which says that the functors between DG derived categories are represented by objects on the product.

Theorem 9.10 ([To], Th.8.9). Let $X$ and $Y$ be quasi-compact and separated schemes over a field $k$. Then we have a canonical quasi-equivalence

$$
\mathcal{D}_{d g}(\mathrm{Q} \operatorname{coh}(X \times Y)) \stackrel{\sim}{\longrightarrow} \mathcal{R} \mathcal{H} \operatorname{mom}_{c}\left(\mathcal{D}_{d g}(\mathrm{Q} \operatorname{coh} X), \mathcal{D}_{d g}(\mathrm{Q} \operatorname{coh} Y)\right),
$$

where $\mathcal{R} \mathcal{H o m}_{c}$ denotes the $D G$ category formed by the direct sums preserving quasifunctors (we say that a quasi-functor preserves direct sums if its homotopy functor does).

Remark 9.11. In other words this theorem tells us that for any quasi-functor $\mathcal{F}$ from $\mathcal{D}_{d q}($ Qcoh $X)$ to $\mathcal{D}_{d q}($ Qcoh $Y)$ that preserves direct sums, the functor $H^{0}(\mathcal{F})$ can be represented by an object on the product $X \times Y$; i.e., it is isomorphic to a functor of the form

$$
\Phi_{\mathcal{E}} \cdot(-):=\mathbf{R} p_{2 *}\left(\mathcal{E} \cdot \stackrel{\mathrm{L}}{\otimes} p_{1}^{*}(-)\right),
$$

where $\mathcal{E}$ is a complex of quasi-coherent sheaves on the product $X \times Y$, and this representation is unique up to isomorphism in $D(\mathrm{Q} \operatorname{coh}(X \times Y))$ (see [To, Cor.8.12).

Combining Theorem 9.10 with the uniqueness of enhancement for the derived category of quasi-coherent sheaves on a quasi-compact and quasi-separated scheme with enough locally free sheaves we obtain the following corollary.

Corollary 9.12. Let $X$ and $Y$ be quasi-compact separated schemes over a field $k$. Assume that $X$ has enough locally free sheaves. Let $F: D(Q \operatorname{coh} X) \rightarrow D(Q \operatorname{coh} Y)$ be a fully faithful functor that commutes with direct sums. Then there is an object $\mathcal{E} \cdot \in D(\mathrm{Q} \operatorname{coh}(X \times Y))$ such that the functor $\Phi_{\mathcal{E}}$. is fully faithful and $\Phi_{\mathcal{E}} \cdot\left(C^{\cdot}\right) \cong$ $F\left(C^{\cdot}\right)$ for any $C^{\cdot} \in D(\mathrm{Q} \operatorname{coh} X)$.

Proof. We know that by Corollary 7.6, $D(\mathrm{Q} \operatorname{coh} X)$ has a unique enhancement. Moreover, by Theorem 7.4 and Lemma 7.2 the category $D(Q \operatorname{coh} X)$ can be represented as a quotient $D(\mathcal{A}) / L$, where $\mathcal{A}$ is a small category formed by a set of locally free sheaves of finite type and $L$ is a localizing subcategory in $D(\mathcal{A})$. Thus, the enhancement $\mathcal{D}_{d g}(\mathrm{Q} \operatorname{coh} X)$ is equivalent to the enhancement $\mathcal{M o d}-\mathcal{A} / \mathcal{L}$. Under the quotient functor, all objects of the form $h^{Y}$ go to locally free sheaves of finite type and, hence, they are compact in $D(\mathrm{Q} \operatorname{coh} X)$. Now we can apply Theorem 5.4 and obtain a quasi-functor $\mathcal{F}$ from $\mathcal{D}_{d g}(\mathrm{Q} \operatorname{coh} X)$ to $\mathcal{D}_{d g}(\mathrm{Qcoh} Y)$ such that $H^{0}(\mathcal{F})$ is fully faithful and $H^{0}(\mathcal{F})\left(C^{\cdot}\right) \cong F\left(C^{\cdot}\right)$ for any $C^{\cdot} \in D($ Qcoh $X)$.

By construction, the functor $H^{0}(\mathcal{F})$ realizes an equivalence of $D($ Qcoh $X)$ with a subcategory in $D(\mathrm{Q} \operatorname{coh} Y)$ that is the essential image of the functor $F$. The inclusion of this subcategory in $D($ Qcoh $Y)$ commutes with direct sums by assumption. Therefore, the functor $H^{0}(\mathcal{F})$ commutes with direct sums and by Theorem 9.10 it has the form $\Phi_{\mathcal{E}}$. for some $\mathcal{E} \cdot D(\mathrm{Q} \operatorname{coh}(X \times Y))$.

Note that under the assumptions in the corollary above the functor $F$ has a right adjoint functor by the Brown representability theorem (see [N3], Thm. 8.4.4).

Corollary 9.13. Let $X$ be a quasi-projective scheme and $Y$ be a quasi-compact and separated scheme. Let $K: \operatorname{Perf}(X) \rightarrow D(\mathrm{Qcoh} Y)$ be a fully faithful functor. 
Then there is an object $\mathcal{E} \in D(\mathrm{Q} \operatorname{coh}(X \times Y))$ such that

(1) the functor $\left.\Phi_{\mathcal{E}} \cdot\right|_{\operatorname{Perf}(X)}: \operatorname{Perf}(X) \rightarrow D(\mathrm{Qcoh} Y)$ is fully faithful and $\Phi_{\mathcal{E}} \cdot\left(P^{\cdot}\right)$ $\cong K\left(P^{\cdot}\right)$ for any $P^{\cdot} \in \operatorname{Perf}(X)$;

(2) if $X$ is projective with $T_{0}\left(\mathcal{O}_{X}\right)=0$, then $\left.\Phi_{\mathcal{E}} \cdot\right|_{\operatorname{Perf}(X)} \cong K$;

(3) if $K$ sends $\operatorname{Perf}(X)$ to $\operatorname{Perf}(Y)$, then the functor $\Phi_{\mathcal{E}}: D(Q \operatorname{coh} X) \rightarrow$ $D(\mathrm{Qcoh} Y)$ is fully faithful and also sends $\operatorname{Perf}(X)$ to $\operatorname{Perf}(Y)$;

(4) if $Y$ is Noetherian and $K$ sends $\operatorname{Perf}(X)$ to $D^{b}(\mathrm{Q} \operatorname{coh} Y)_{\operatorname{coh}}$, then the object $\mathcal{E} \cdot$ is isomorphic to an object of $D^{b}(\operatorname{coh}(X \times Y))$.

Proof. Since $X$ is a quasi-projective scheme it is an open subscheme of a projective scheme $\bar{X} \subset \mathbb{P}^{n}$. We have a graded $A$ algebra $A=\bigoplus_{n} H^{0}\left(\bar{X}, \mathcal{O}_{\bar{X}}(n)\right)$ and a corresponding $\mathbb{Z}$-category $\mathcal{A}$, objects of which are $\mathbb{Z}$ and morphisms $\operatorname{Hom}_{\mathcal{A}}(i, j)=$ $A^{j-i}$.

As was explained in Section 7 the category $D($ Qcoh $X)$ is equivalent to the quotient $D(\mathcal{A}) / D_{I}(\mathcal{A})$, where $D_{I}(\mathcal{A})$ is a localizing subcategory of complexes with $I$-torsion cohomologies (here $I$ is an ideal such that the support of the subscheme $\mathbb{P} \operatorname{roj} A / I \subset \bar{X}$ is exactly $\bar{X} \backslash X)$. In addition, the free modules $h^{Y}$ map to the corresponding line bundles $\left.\mathcal{O}(i)\right|_{X}$ which are compact objects in $D($ Qcoh $X)$, and $\operatorname{Perf}(X) \cong\left(D(\mathcal{A}) / D_{I}(\mathcal{A})\right)^{c}$.

As we know (see Proposition 1.17) the DG categories $\mathcal{D}_{d g}(\mathrm{Qcoh} X)$ and $\mathcal{D}_{d g}($ Qcoh $X)$ are quasi-equivalent to the DG categories $\mathcal{S} \mathcal{F}(\mathcal{P} \operatorname{erf}(X))$ and $\mathcal{S F}(\mathcal{P} \operatorname{erf}(Y))$, respectively, and we denote by $\phi_{X}$ and $\phi_{Y}$ the corresponding quasifunctors. For shortness, denote by $\mathcal{C}$ the DG category $\operatorname{Per} f(Y)$ and by $\mathcal{C}^{\prime}$ the full DG subcategory in $\mathcal{S F}(\mathcal{C}) \cong \mathcal{D}_{d g}($ Qcoh $Y)$ which consists of all objects in the essential image of $H^{0}\left(\phi_{Y}\right) K$. The functor $K$ induces an equivalence $N: \operatorname{Perf}(X) \stackrel{\sim}{\rightarrow}$ $H^{0}\left(\mathcal{C}^{\prime}\right)$.

By Theorem 6.4 there is a quasi-functor $\mathcal{N}: \mathcal{P} \operatorname{erf}(X) \rightarrow \mathcal{C}^{\prime}$ which is a quasiequivalence. It induces a quasi-equivalence $\mathcal{N}^{*}: \mathcal{S F}(\mathcal{P} \operatorname{erf}(X)) \rightarrow \mathcal{S F}\left(\mathcal{C}^{\prime}\right)$.

Let $\mathcal{D}$ be a DG subcategory $\mathcal{D} \subset \mathcal{S F}(\mathcal{C})$ that contains $\mathcal{C}$ and $\mathcal{C}^{\prime}$. We denote by $\mathcal{J}: \mathcal{C}^{\prime} \hookrightarrow \mathcal{D} \subset \mathcal{S F}(\mathcal{C})$ and $\mathcal{I}: \mathcal{C} \hookrightarrow \mathcal{D} \subset \mathcal{S F}(\mathcal{C})$ the respective DG full embeddings. We have the extension DG functor $\mathcal{J}^{*}: \mathcal{S F}\left(\mathcal{C}^{\prime}\right) \rightarrow \mathcal{S F}(\mathcal{D})$ and the restriction DG functor $\mathcal{I}_{*}: \operatorname{Mod}-\mathcal{D} \rightarrow \operatorname{Mod}-\mathcal{C}$, which induces a quasi-functor $\iota_{*}: \mathcal{S F}(\mathcal{D}) \rightarrow$ $\mathcal{S F}(\mathcal{C})$. Consider the composition of quasi-functors $\iota_{*} \cdot \mathcal{J}^{*} \cdot \mathcal{N}^{*}: \mathcal{S F}(\mathcal{P} \operatorname{erf}(X)) \rightarrow$ $\mathcal{S F}(\mathcal{P} \operatorname{erf}(Y))$. The functors $H^{0}\left(\iota_{*}\right), H^{0}\left(\mathcal{J}^{*}\right)$, and $H^{0}\left(\mathcal{N}^{*}\right)$ evidently commute with direct sums. (Note that the right adjoint to the quasi-functor $\iota_{*} \mathcal{J}^{*}$ is the quasifunctor $\phi: \mathcal{S F}(\mathcal{C}) \rightarrow \mathcal{S F}\left(\mathcal{C}^{\prime}\right)$ which is induced by the DG functor $\Phi: \mathcal{S F}(\mathcal{C}) \rightarrow$ $\mathcal{M o d}-\mathcal{C}^{\prime}$ given by the standard formula $\Phi(M)\left(C^{\prime}\right)=\operatorname{Hom}_{\mathcal{S F}(\mathcal{C})}\left(C^{\prime}, M\right)$ for $C^{\prime} \in$ $\mathcal{C}^{\prime}, M \in \mathcal{S F}(\mathcal{C})$.)

Thus, considering the compositions of quasi-functors $\phi_{Y}^{-1} \cdot \iota_{*} \cdot \mathcal{J}^{*} \cdot \mathcal{N}^{*} \cdot \phi_{X}$ we obtain a quasi-functor $\mathcal{F}$ from $\mathcal{D}_{d g}(\mathrm{Qcoh} X)$ to $\mathcal{D}_{d g}(\mathrm{Qcoh} Y)$ that completes the following commutative diagram:

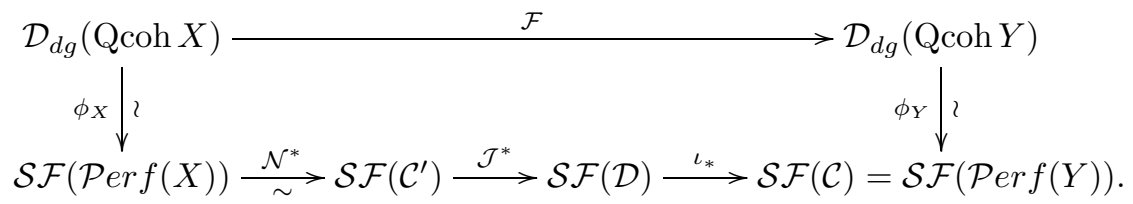

The quasi-functor $\mathcal{F}$ commutes with direct sums and, hence, by Theorem 9.10 the functor $H^{0}(\mathcal{F})$ is isomorphic to $\Phi_{\mathcal{E}}$. with $\mathcal{E} \in D(\mathrm{Q} \operatorname{coh}(X \times Y))$. 
(1) The restriction of the quasi-functor $\iota_{*} \mathcal{J}^{*}$ on $\mathcal{C}^{\prime}$ is isomorphic to the inclusion $\mathcal{C}^{\prime} \rightarrow \mathcal{S F}(\mathcal{C})$. This implies that the restriction $\left.\Phi_{\mathcal{E}} \cdot\right|_{\operatorname{Perf}(X)}$ is fully faithful. Moreover, by property (3) of Theorem 6.4 there is an isomorphism $H^{0}(\mathcal{N})\left(P^{\cdot}\right) \cong N\left(P^{\cdot}\right)$ for any $P^{\cdot} \in \operatorname{Perf}(X)$. Hence, $\Phi_{\mathcal{E}^{\cdot}}\left(P^{\cdot}\right) \cong K\left(P^{\cdot}\right)$.

(3) If $K$ sends $\operatorname{Perf}(X)$ to $\operatorname{Perf}(Y)$, then we can take $\mathcal{D}=\mathcal{C}$. In this case, $\iota_{*}$ is the identity, but $H^{0}\left(\mathcal{J}^{*}\right)$ is fully faithful by Proposition 1.15 . Therefore, $H^{0}(\mathcal{F})$ is fully faithful and sends $\operatorname{Perf}(X)$ to $\operatorname{Perf}(Y)$.

(2) Let $X$ be projective. We know by property (2) of Theorem 6.4 that there is an isomorphism of functors $\theta: H^{0}(\mathcal{N}) \cdot \pi \cdot h^{\bullet} \stackrel{\sim}{\rightarrow} N \cdot \pi \cdot h^{\bullet}$ from $\mathcal{A}$ to $H^{0}\left(\mathcal{C}^{\prime}\right)$.

Consider the composition $G=H^{0}(\mathcal{N})^{-1} \cdot N$ from $\operatorname{Perf}(X)$ to itself. We have a natural transformation from $j \stackrel{\sim}{\rightarrow} G \cdot j$ on the subcategory $h^{\bullet}(\mathcal{A})$ which coincides with the full subcategory $\mathcal{P}:=\left\{\mathcal{O}_{X}(i)\right\}_{i \in \mathbb{Z}}$. Since $X$ is projective with $T_{0}\left(\mathcal{O}_{X}\right)=0$ the sequence $\left\{\mathcal{O}_{X}(i)\right\}_{i \in \mathbb{Z}}$ is ample in $\operatorname{Loc} X$. Hence we can apply Proposition 9.6 to $\operatorname{Perf}(X)$ and obtain that the functor $G$ is isomorphic to the identity functor on the whole $\operatorname{Perf}(X)$. Therefore, the functors $H^{0}(\mathcal{N})$ and $N$ are isomorphic; i.e., the functor $K$ is isomorphic to the functor $\left.\Phi_{\mathcal{E}} \cdot\right|_{\operatorname{Perf}(X)}$.

(4) Finally, we have to argue that the object $\mathcal{E}$ is isomorphic to an object from $D^{b}(\operatorname{coh}(X \times Y))$ if $Y$ is Noetherian and the functor $\Phi_{\mathcal{E}}$ sends $\operatorname{Perf}(X)$ to $D^{b}(\mathrm{Q} \operatorname{coh} Y)_{\text {coh }}$. It is well known that the canonical functor $D^{b}(\operatorname{coh} Z) \rightarrow$ $D(\mathrm{Qcoh} Z)$ is fully faithful and establishes an equivalence with the subcategory $D^{b}(\text { Qcoh } Z)_{\text {coh }}$ of cohomologically bounded complexes with coherent cohomologies. Consider an inclusion $i: X \hookrightarrow \mathbb{P}^{N}$. The composition of the inverse image functor $\mathbf{L} i^{*}$ and $\Phi_{\mathcal{E}}$. gives a functor from $D\left(\mathrm{Qcoh} \mathbb{P}^{N}\right)$ to $D(\mathrm{Qcoh} Y)$ which is represented by the object $\mathbf{R}\left(i, \mathrm{id}_{Y}\right)_{*} \mathcal{E}$ and sends $\operatorname{Perf}\left(\mathbb{P}^{N}\right)$ to $D^{b}(\mathrm{Qcoh} Y)_{\text {coh }}$. It is sufficient to check that the object $\mathbf{R}\left(i, \mathrm{id}_{Y}\right)_{*} \mathcal{E}$ belongs to $D^{b}\left(\mathrm{Q} \operatorname{coh}\left(\mathbb{P}^{N} \times Y\right)\right)_{\text {coh }}$. The category $D\left(\mathrm{Q} \operatorname{coh}\left(\mathbb{P}^{N} \times Y\right)\right)$ has a semi-orthogonal decomposition of the form $\langle\mathcal{O}(-N) \otimes D(\mathrm{Q} \operatorname{coh} Y), \ldots, \mathcal{O} \otimes D(\mathrm{Q} \operatorname{coh} Y)\rangle$. The components of $\mathbf{R}\left(i, \mathrm{id}_{Y}\right)_{*} \mathcal{E}$ with respect to this decomposition are isomorphic to $\mathcal{O}(-p) \otimes \Phi_{\mathcal{E}} \cdot \mathbf{L} i^{*}\left(\Omega_{\mathbb{P} N}^{p}[p]\right)$.

There is a similar decomposition $\left\langle\mathcal{O}(-N) \otimes D^{b}(\operatorname{coh} Y), \ldots, \mathcal{O} \otimes D^{b}(\operatorname{coh} Y)\right\rangle$ for the bounded category of coherent sheaves $D^{b}\left(\operatorname{coh}\left(\mathbb{P}^{N} \times Y\right)\right)$. Since the objects $\Phi_{\mathcal{E}} \mathbf{L} i^{*}\left(\Omega_{\mathbb{P}^{N}}^{p}[p]\right)$ belong to $D^{b}(\mathrm{Q} \operatorname{coh} Y)_{\text {coh }}$, the object $\mathbf{R}\left(i, \mathrm{id}_{Y}\right)_{*} \mathcal{E}$ is isomorphic to an object of $D^{b}\left(\operatorname{coh}\left(\mathbb{P}^{N} \times Y\right)\right)$. Therefore, the object $\mathcal{E}$ is also isomorphic to an object of $D^{b}(\operatorname{coh}(X \times Y))$.

Let us consider the bounded derived category of coherent sheaves $D^{b}(\operatorname{coh} X)$ on a quasi-projective scheme $X$. Consider an exact functor $F$ from $D^{b}(\operatorname{coh} X)$ to a triangulated category $\mathcal{T}$ that admits arbitrary direct sums. We say that the functor $F$ commutes with homotopy colimits if for any $M \in D^{b}(\operatorname{coh} X)$, which is isomorphic in $D(\mathrm{Q} \operatorname{coh} X)$ to $\underline{\operatorname{hocolim}}_{i} P_{i}$ of perfect complexes $P_{i}^{*}$, there is an isomorphism $F(M) \stackrel{\sim}{\rightarrow} \operatorname{hocolim}_{i} F\left(P_{i}^{*}\right)$ in $\mathcal{T}$ that commutes with canonical morphisms from $F\left(P_{i}\right)$ for each $i$. Note that our functor $F$ is defined only on $D^{b}(\operatorname{coh} X) \cong D^{b}(\mathrm{Q} \operatorname{coh} X)_{\operatorname{coh}} \subset D(\mathrm{Q} \operatorname{coh} X)$.

Corollary 9.14. Let $X$ be a projective scheme with $T_{0}\left(\mathcal{O}_{X}\right)=0$ and $Y$ be a quasi-compact and separated scheme. Let $K: D^{b}(\operatorname{coh} X) \rightarrow D(\mathrm{Q} \operatorname{coh} Y)$ be a fully faithful functor that commutes with homotopy colimits. Then there is an object $\mathcal{E} \in D(\mathrm{Q} \operatorname{coh}(X \times Y))$ such that $\left.\Phi_{\mathcal{E}} \cdot\right|_{D^{b}(\operatorname{coh} X)} \cong K$. 
Proof. We can consider the restriction of the functor $K$ on the subcategory of perfect complexes $\operatorname{Perf}(X) \subset D^{b}(\mathrm{Q} \operatorname{coh} X)_{\mathrm{coh}} \cong D^{b}(\operatorname{coh} X)$. By Corollary 9.13 there is an object $\mathcal{E} \in D(\mathrm{Q} \operatorname{coh}(X \times Y))$ and an isomorphism of functors $\theta:\left.\Phi_{\mathcal{E}} \cdot\right|_{\operatorname{Perf}(X)} \cong$ $\left.K\right|_{\operatorname{Perf}(X)}$. For any object $M \in D^{b}(\operatorname{coh} X)$ there is a quasi-isomorphism $P^{\cdot} \stackrel{\sim}{\rightarrow}$ $M^{*}$, where $P^{\cdot}$ is a bounded above complex of locally free sheaves of finite type (see Lemma 8.11). Hence, the object $M^{\cdot}$ is isomorphic to hocolim ${ }_{k} \sigma_{\geq k} P^{*}$ in $D($ Qcoh $X)$. By assumption, there is an isomorphism ${\underset{\text { hocolim}}{\longrightarrow}}_{k} K\left(\sigma_{\geq k} P^{\cdot}\right) \stackrel{\sim}{\rightarrow} K\left(M^{\cdot}\right)$ in $D(\mathrm{Q} \operatorname{coh} Y)$. On the other hand, the functor $\Phi_{\mathcal{E}}$ commutes with direct sums and homotopy colimits. Therefore, there is an isomorphism $\theta_{M}: \Phi_{\mathcal{E}} \cdot\left(M^{*}\right) \stackrel{\sim}{\rightarrow} K\left(M^{*}\right)$ that makes the following square

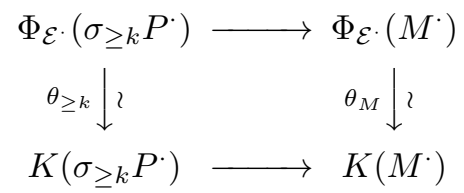

commutative for any $k$.

Now we have to check that the restriction of $\Phi_{\mathcal{E}}$. on $D^{b}(\operatorname{coh} X)$ is fully faithful. Let $Q$ belong to $\operatorname{Perf}(X)$. Any morphism $f: Q \rightarrow M$ factors through $\sigma_{\geq k} P^{\cdot}$ for $k \ll 0$. Since the right and left squares in the following diagram

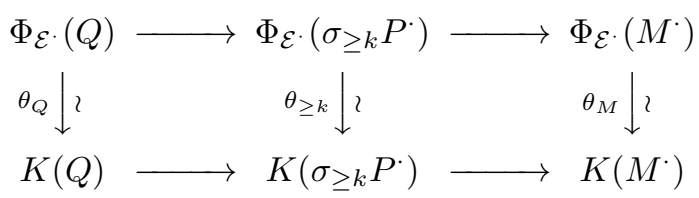

commute, the outside square also commutes. Therefore, $\theta_{M} \cdot \Phi_{\mathcal{E}} \cdot(f)=K(f) \cdot \theta_{Q}$ for any $f: Q \rightarrow M$. If now $\Phi_{\mathcal{E}}(f)=0$, then $K(f)=0$ and $f=0$, because $K$ is fully faithful. On the other hand, for any $g: \Phi_{\mathcal{E}}(Q) \rightarrow \Phi_{\mathcal{E}}\left(M^{\cdot}\right)$ we can consider $g^{\prime}=\theta_{M} \cdot g \cdot \theta_{Q}^{-1}$ and take $f: Q \rightarrow M \cdot$ such that $K(f)=g^{\prime}$. It is obvious now that $\Phi_{\mathcal{E}} \cdot(f)=g$. Thus, there are isomorphisms

$$
\operatorname{Hom}\left(Q, M^{\cdot}\right) \stackrel{\sim}{\longrightarrow} \operatorname{Hom}\left(\Phi_{\mathcal{E}} \cdot(Q), \Phi_{\mathcal{E}} \cdot\left(M^{\cdot}\right)\right)
$$

for any $Q \in \operatorname{Perf}(X)$ and every $M \cdot \in D^{b}(\operatorname{coh} X)$.

The objects $C^{\cdot} \in D(\mathrm{Q} \operatorname{coh} X)$ for which the natural map

$$
\operatorname{Hom}\left(C^{\cdot}, M^{\cdot}[m]\right) \stackrel{\sim}{\longrightarrow} \operatorname{Hom}\left(\Phi_{\mathcal{E}}\left(C^{\cdot}\right), \Phi_{\mathcal{E}}\left(M^{\cdot}\right)[m]\right)
$$

is bijective for all $m \in \mathbb{Z}$ form a triangulated subcategory in $D(\mathrm{Qcoh} X)$ which contains $\operatorname{Perf}(X)$ and is closed under direct sums (since $\Phi_{\mathcal{E}}$. commutes with direct sums). Therefore, this subcategory coincides with the whole $D(\mathrm{Qcoh} X)$. Thus, the functor $\left.\Phi_{\mathcal{E}}\right|_{D^{b}(\operatorname{coh} X)}$ is fully faithful and has the same essential image as $K$. Now we can apply Proposition 9.6 and extend the isomorphism $\theta:\left.\left.\Phi_{\mathcal{E}} \cdot\right|_{\operatorname{Perf}(X)} \cong K\right|_{\operatorname{Perf}(X)}$ to an isomorphism of the functors $\left.\Phi_{\mathcal{E}} \cdot\right|_{D^{b}(\operatorname{coh} X)} \cong K$.

Remark 9.15. Notice that in the proof of the corollary we used only the fact that $K$ commutes with homotopy colimits of a special form hocolim $\sigma_{\geq k} P$ and this implies that $\left.K \cong \Phi_{\mathcal{E}}\right|_{D^{b}(\operatorname{coh} X)}$. Thus, as a result, it commutes with all homotopy colimits.

Example 9.16. We say that the functor $K: D^{b}(\operatorname{coh} X) \rightarrow D(\mathrm{Q} \operatorname{coh} Y)$ is bounded above by $n \in \mathbb{Z}$ if $H^{i}(K(\mathcal{F}))=0$ for all coherent sheaves $\mathcal{F} \in \operatorname{coh} X$ when $i>n$. If the functor $K$ is bounded above, then it commutes with homotopy colimits. Indeed, 
for any $M^{\cdot} \in D^{b}(\operatorname{coh} X)$ and a locally free resolution $P^{\cdot} \rightarrow M^{\cdot}$ we have that the map $K\left(\sigma_{\geq k} P^{\cdot}\right) \rightarrow K\left(M^{\cdot}\right)$, when $k \ll 0$, is an isomorphism on cohomologies $H^{i}$ for all $i>k+n$. Therefore, hocolim$_{k} K\left(\sigma_{\geq k} P^{\cdot}\right) \cong K\left(M^{\cdot}\right)$. By the remark above, $K$ commutes with homotopy colimits.

Corollary 9.17. Let $X$ be a projective scheme with $T_{0}\left(\mathcal{O}_{X}\right)=0$ and $Y$ be a Noetherian scheme. Let $K: D^{b}(\operatorname{coh} X) \rightarrow D^{b}(\operatorname{coh} Y)$ be a fully faithful functor that has a right adjoint $K^{!}: D^{b}(\operatorname{coh} Y) \rightarrow D^{b}(\operatorname{coh} X)$. Then there is an object $\mathcal{E} \cdot \in D^{b}(\operatorname{coh}(X \times Y))$ such that $\left.\Phi_{\mathcal{E}}\right|_{D^{b}(\operatorname{coh} X)} \cong K$.

Proof. To apply Corollary 9.14 we need to check that the functor $K$ commutes with homotopy colimits. Following the example above it is sufficient to show that the functor $K$ is bounded above. First, we easily see that $K$ is bounded above on the sequence of line bundles $\{\mathcal{O}(j)\}_{j \in \mathbb{Z}}$, because by Beilinson's theorem for any $j>0$ (resp. $j<-N$ ) we have a left (resp. right) resolution of $\mathcal{O}_{X}(j)$ of the form

$$
V_{N} \otimes \mathcal{O}_{X}(-N) \longrightarrow \cdots \longrightarrow V_{1} \otimes \mathcal{O}_{X}(-1) \longrightarrow V_{0} \otimes \mathcal{O}_{X},
$$

where $X \hookrightarrow \mathbb{P}^{N}$ is a closed embedding and $V_{s}$ is $H^{0}\left(\mathbb{P}^{N}, \Omega^{s}(s+j)\right)\left(\right.$ resp. $H^{N}\left(\mathbb{P}^{N}\right.$, $\left.\Omega^{s}(s+j)\right)$ ) (see, for example, OSS, Th.3.1.4).

Denote by $n$ an integer such that $H^{i}(K(\mathcal{O}(j))=0$ for all $j$ when $i>n$. If $\mathcal{F} \in$ $\operatorname{coh} X$ is a coherent sheaf that has a highest nontrivial cohomology $H^{m}(K(\mathcal{F}))=$ $\mathcal{G} \neq 0$ with $m>n$, then we can construct a sequence of coherent sheaves $\left\{\mathcal{F}_{p} \in\right.$ $\operatorname{coh} X\}_{p \geq 0}$ such that $H^{m+p}\left(K\left(\mathcal{F}_{p}\right)\right)=\mathcal{G}$. The construction goes by induction with base $\mathcal{F}_{0}=\mathcal{F}$ and $\mathcal{F}_{p+1}$ is the kernel of some epimorphism from $\mathcal{O}\left(-k_{p}\right)^{\oplus n_{p}}$ to $\mathcal{F}_{p}$. It is evident that such an epimorphism exists when $k_{p} \ll 0$ and the highest cohomology of $K\left(\mathcal{F}_{p+1}\right)$ has a number $m+p+1$ and it is isomorphic to the $H^{m+p}\left(K\left(\mathcal{F}_{p}\right)\right) \cong \mathcal{G}$.

Assume that $K$ has a right adjoint functor $K^{!}: D^{b}(\operatorname{coh} Y) \rightarrow D^{b}(\operatorname{coh} X)$. Take the object $K^{!}(\mathcal{G})$. By construction above we have that

$$
\operatorname{Hom}\left(\mathcal{F}_{p}, K^{!}(\mathcal{G})[-m-p]\right) \cong \operatorname{Hom}\left(K\left(\mathcal{F}_{p}\right), \mathcal{G}[-m-p]\right) \neq 0
$$

for all $p \geq 0$. This gives a contradiction with the fact that $K^{!}(\mathcal{G})$ is a bounded complex. Therefore, $K$ is bounded above and by Example 9.16 and Corollary 9.14 it is isomorphic to the functor $\Phi_{\mathcal{E}}$. for some $\mathcal{E} \in D(\mathrm{Q} \operatorname{coh}(X \times Y))$. Since the functor $K$ sends $\operatorname{Perf}(X)$ to $D^{b}(\operatorname{coh} Y)$, Corollary 9.13 (4) implies that $\mathcal{E}$ is isomorphic to an object of $D^{b}(\operatorname{coh}(X \times Y))$.

Remark 9.18. The last statement also holds under the conditions that $Y$ is projective and $K$ has a partially left adjoint $K^{*}: \operatorname{Perf}(Y) \rightarrow \operatorname{Perf}(X)$. In this case, for sufficiently large $s \gg 0$, there is a nontrivial morphism from $\mathcal{O}_{Y}(-s)$ to $\mathcal{G}$ that can be lifted to a nontrivial morphism from $\mathcal{O}_{Y}(-s)$ to $K\left(\mathcal{F}_{p}[m+p]\right)$. Therefore,

$$
\operatorname{Hom}\left(K^{*}\left(\mathcal{O}_{Y}(-s)\right), \mathcal{F}_{p}[m+p]\right) \cong \operatorname{Hom}\left(\mathcal{O}_{Y}(-s), K\left(\mathcal{F}_{p}\right)[m+p]\right) \neq 0 .
$$

This gives a contradiction with the fact that $K^{*}\left(\mathcal{O}_{Y}(-s)\right)$ is a perfect complex, and, hence, $K$ is bounded above as well.

\section{Appendix A. Small U-cocomplete Categories}

Fix universes $\mathbb{U} \in \mathbb{V}$, so that $\mathbb{U}$ contains an infinite set [SGA4]. A set $X$ is called a $\mathbb{U}$-set (resp. $\mathbb{U}$-small) if $X \in \mathbb{U}$ (resp. $X$ is isomorphic to an element of $\mathbb{U}$ ). Similarly for groups, vector spaces, etc.

We call $\mathcal{C}$ a $\mathbb{U}$-category if for each $A, B \in \mathcal{C}$, the set $\operatorname{Hom}(A, B)$ is $\mathbb{U}$-small. We call a $\mathbb{U}$-category (or a DG $\mathbb{U}$-category) $\mathcal{C} \mathbb{U}$-small if the collection of objects of $\mathcal{C}$ 
is a $\mathbb{U}$-small set. Also a $\mathbb{U}$-category is called essentially $\mathbb{U}$-small if the isomorphism classes of its objects form a $\mathbb{U}$-small set. Denote by $\mathcal{A} b_{\mathbb{U}}$ the category of $\mathbb{U}$-small abelian groups.

A triangulated $\mathbb{U}$-category is called $\mathbb{U}$-cocomplete if it has all $\mathbb{U}$-small direct sums, i.e., if it has a direct sum of any collection of its objects which is indexed by a U-small set.

We would like to translate some well-known fact about cocomplete triangulated categories into the language of triangulated $\mathbb{U}$-categories which are $\mathbb{U}$-cocomplete.

Fix a triangulated $\mathbb{U}$-category $\mathcal{T}$ which is $\mathbb{U}$-cocomplete. Recall that a set $S \subset \mathcal{T}$ generates $\mathcal{T}$ if whenever $\operatorname{Hom}(A, X[n])=0$ for all $A \in S$ and all $n \in \mathbb{Z}$, then $X=0$. For a subset $S \subset \mathcal{T}$ denote by $\langle S\rangle^{\mathbb{U}}$ the smallest strictly full triangulated subcategory which contains $S$ and is $\mathbb{U}$-cocomplete.

Theorem A.1 (Brown representability for $\mathbb{U}$-categories). Let $\mathcal{T}$ be a triangulated $\mathbb{U}$-category $\mathcal{T}$ which is $\mathbb{U}$-cocomplete. Suppose that $\mathcal{T}$ is compactly generated by a $\mathbb{U}$-small set $S \subset \mathcal{T}^{c}$.

a) Let $H: \mathcal{T}^{\mathrm{op}} \rightarrow \mathcal{A} b_{\mathbb{U}}$ be a cohomological functor which takes $\mathbb{U}$-small coproducts to products. Then $H$ is representable.

b) $\mathcal{T}=\langle S\rangle^{\mathbb{U}}$.

Proof. The (simultaneous) proof is the same as in the book of A. Neeman [N3], Theorem 8.3.3.

Lemma A.2. Let $\mathcal{T}$ be a triangulated $\mathbb{U}$-category. Assume that $\mathcal{S} \subset \mathcal{T}$ is an essentially $\mathbb{U}$-small triangulated subcategory. Then the Verdier quotient $\mathcal{T} / \mathcal{S}$ is a $\mathbb{U}$-category.

Proof. The categories $\mathcal{T}$ and $\mathcal{T} / \mathcal{S}$ have the same objects. Fix objects $A$ and $B$. A morphism $f$ between $A$ and $B$ in $\mathcal{T} / \mathcal{S}$ is represented by a diagram $A \stackrel{s}{\leftarrow} C \stackrel{g}{\rightarrow} B$, where the cone $D$ of the morphism $s$ is in $\mathcal{S}$. Up to isomorphism we have a $\mathbb{U}$-small set of choices for a diagram $A \rightarrow D$, where $D \in \mathcal{C}$. Thus up to isomorphism we have a U-small set of choices for a diagram $A \stackrel{s}{\leftarrow} C$, such that the cone of $s$ is in $\mathcal{S}$. Thus up to isomorphism there is a U-small set of choices for a diagram $A \stackrel{s}{\leftarrow} C \stackrel{g}{\rightarrow} B$ as above.

Lemma A.3. Let $\mathcal{T}$ be a triangulated category which is $\mathbb{U}$-cocomplete. Assume that $\mathcal{S} \subset \mathcal{T}$ is a full triangulated subcategory which is closed under $\mathbb{U}$-small direct sums in $\mathcal{T}$. Then the quotient category $\mathcal{T} / \mathcal{S}$ is also $\mathbb{U}$-cocomplete and the functor $\pi: \mathcal{T} \rightarrow \mathcal{T} / \mathcal{S}$ preserves $\mathbb{U}$-small direct sums.

Proof. The proof is the same as the proof of Lemma 3.2.10 in [N3].

Remark A.4. Note that in the last lemma we do not know if the quotient $\mathcal{T} / \mathcal{S}$ is a $\mathbb{U}$-category, or even if morphisms between two objects in $\mathcal{T} / \mathcal{S}$ form a set.

Proposition A.5. Let $\mathcal{T}$ be a triangulated $\mathbb{U}$-category which is $\mathbb{U}$-cocomplete. Let $\mathcal{S} \subset \mathcal{T}$ be a full triangulated subcategory which is closed under $\mathbb{U}$-small direct sums in $\mathcal{T}$. Assume that $\mathcal{S}$ is generated by a $\mathbb{U}$-small set of objects in $\mathcal{S} \cap \mathcal{T}^{c}$. Then

a) the localization functor $\pi: \mathcal{T} \rightarrow \mathcal{T} / \mathcal{S}$ has a right adjoint functor $\mu$ which is full and faithful;

b) $\mu$ preserves arbitrary direct sums;

c) $\mathcal{T} / \mathcal{S}$ is a $\mathbb{U}$-category. 
Proof. c) is an immediate consequence of a). The proofs of a), b) are the same as in [N1, Lemma 1.7, Proposition 1.9] and [N3, Lemma 9.1.7].

Theorem A.6. Let $\mathcal{T}$ be a triangulated $\mathbb{U}$-category which is $\mathbb{U}$-cocomplete and such that the category $\mathcal{T}^{c}$ is essentially $\mathbb{U}$-small. Let $\mathcal{S} \subset \mathcal{T}$ be a full triangulated subcategory which is closed under $\mathbb{U}$-small direct sums in $\mathcal{T}$. Assume that $\mathcal{S}$ is generated by a $\mathbb{U}$-small set of objects in $\mathcal{S} \cap \mathcal{T}^{c}$. Let $\mathcal{C}=\mathcal{T} / \mathcal{S}$ be the quotient category. Then

(1) $\mathcal{C}$ is generated by a $\mathbb{U}$-small subset of its compact objects;

(2) $\mathcal{T}^{c}$ maps to $\mathcal{C}^{c}$ under the quotient functor;

(3) the induced functor $\mathcal{T}^{c} / \mathcal{S}^{c} \rightarrow \mathcal{C}^{c}$ is full and faithful;

(4) $\mathcal{C}^{c}$ is the idempotent completion of $\mathcal{T}^{c} / \mathcal{S}^{c}$.

Proof. The proof is the same as the proof of Theorem 2.1 in N1.

Let $\mathcal{A}$ be a $\mathbb{U}$-small DG $\mathbb{U}$-category. Denote by $\mathcal{M o d}_{\mathbb{U}}-\mathcal{A} \subset \mathcal{M}$ od $-\mathcal{A}$ the full DG subcategory consisting of all $\mathbb{U}$-small DG $\mathcal{A}$-modules, i.e., DG modules $M$ such that $M(Y)$ is a $\mathbb{U}$-small vector space for each $Y \in \mathcal{A}$. It is a pretriangulated category. Put

$$
\mathcal{S} \mathcal{F}_{\mathbb{U}}(\mathcal{A})=\mathcal{S F}(\mathcal{A}) \cap \mathcal{M} o d_{\mathbb{U}}-\mathcal{A}, \quad \mathcal{A} c_{\mathbb{U}}(\mathcal{A})=\mathcal{A} c(\mathcal{A}) \cap \mathcal{M} o d_{\mathbb{U}}-\mathcal{A}
$$

Let $D_{\mathbb{U}}(\mathcal{A})=H^{0}\left(\mathcal{M o d} d_{\mathbb{U}}-\mathcal{A}\right) / H^{0}\left(\mathcal{A} c_{\mathbb{U}}(\mathcal{A})\right)$ be the corresponding derived category. The natural functor $H^{0}\left(\mathcal{S} \mathcal{F}_{\mathbb{U}}(\mathcal{A})\right) \rightarrow D_{\mathbb{U}}(\mathcal{A})$ is an equivalence of triangulated $\mathbb{U}$ categories. (This is because every $\mathbb{U}$-small $\mathrm{DG} \mathcal{A}$-module is quasi-isomorphic to a $\mathbb{U}$-small semi-free DG module. The proof is the same as in [K1], Thm. $3.1 \mathrm{~b})$.)

Note that triangulated categories $H^{0}\left(\mathcal{M} o d_{\mathbb{U}}-\mathcal{A}\right), H^{0}\left(\mathcal{S} \mathcal{F}_{\mathbb{U}}(\mathcal{A})\right), H^{0}\left(\mathcal{A} c_{\mathbb{U}}(\mathcal{A})\right)$, and $D_{\mathbb{U}}(\mathcal{A})$ are $\mathbb{U}$-cocomplete and since $\mathcal{A}$ is a DG $\mathbb{U}$-category the image of the Yoneda DG functor $h^{\bullet}: \mathcal{A} \rightarrow \operatorname{Mod}-\mathcal{A}$ lies in $\operatorname{Mod}_{\mathbb{U}}-\mathcal{A}$.

Note also that the DG category $\mathcal{M o d}_{\mathbb{U}}-\mathcal{A}$ is DG equivalent to a DG category which is $\mathbb{V}$-small. Indeed, call $M \in \mathcal{M o d}_{\mathbb{U}}-\mathcal{A}$ strict if $M(Y)$ is a $\mathbb{U}$-set (and not just a $\mathbb{U}$-small set). Denote by $\mathcal{M} o d_{\mathbb{U}}^{\text {str }}-\mathcal{A}$ the full DG subcategory consisting of strict DG modules. Clearly every DG module is DG isomorphic to a strict one. The DG category $\mathcal{M} o d_{\mathbb{U}}^{\text {str }}-\mathcal{A}$ is obviously $\mathbb{V}$-small.

Denote $\mathcal{S} \mathcal{F}_{\mathbb{U}}^{\text {str }}(\mathcal{A})=\mathcal{S} \mathcal{F}_{\mathbb{U}}(\mathcal{A}) \cap \mathcal{M} o d_{\mathbb{U}}^{\text {str }}-\mathcal{A}$ and similarly for $\mathcal{A} c_{\mathbb{U}}^{\text {str }}(\mathcal{A})$. The inclusions

$$
\mathcal{M} o d_{\mathbb{U}}^{\mathrm{str}}-\mathcal{A} \subset \mathcal{M} o d_{\mathbb{U}}-\mathcal{A}, \quad \mathcal{S} \mathcal{F}_{\mathbb{U}}^{\mathrm{str}}(\mathcal{A}) \subset \mathcal{S} \mathcal{F}_{\mathbb{U}}(\mathcal{A}), \quad \mathcal{A} c_{\mathbb{U}}^{\mathrm{str}}(\mathcal{A}) \subset \mathcal{A} c_{\mathbb{U}}(\mathcal{A})
$$

are quasi-equivalences of pre-triangulated categories. Hence

$$
H^{0}\left(\mathcal{M o d} \mathbb{U}_{\mathbb{U}}^{\mathrm{str}}-\mathcal{A}\right) / H^{0}\left(\mathcal{A} c_{\mathbb{U}}^{\mathrm{str}}(\mathcal{A})\right) \simeq D_{\mathbb{U}}(\mathcal{A})
$$

and the natural functor $H^{0}\left(\mathcal{S} \mathcal{F}_{\mathbb{U}}^{\operatorname{str}}(\mathcal{A})\right) \rightarrow D_{\mathbb{U}}(\mathcal{A})$ is an equivalence. Thus $\mathcal{S F}_{\mathbb{U}}^{\operatorname{str}}(\mathcal{A})$ is a $\mathbb{V}$-small enhancement of $D_{\mathbb{U}}(\mathcal{A})$. In particular, the $\mathbb{U}$-category $D_{\mathbb{U}}(\mathcal{A})$ is essentially $\mathbb{V}$-small.

Let $L \subset D_{\mathbb{U}}(\mathcal{A})$ be a strictly full triangulated subcategory. Then $L$ is essentially $\mathbb{V}$-small. Hence the quotient $D_{\mathbb{U}}(\mathcal{A}) / L$ is a $\mathbb{V}$-category by Lemma A.2 above.

Let $\mathcal{L} \subset \mathcal{S F}_{\mathbb{U}}^{\operatorname{str}}(\mathcal{A})$ be the full DG subcategory of objects which map to $L$ under the equivalence $H^{0}\left(\mathcal{S} \mathcal{F}_{\mathbb{U}}^{\text {str }}(\mathcal{A})\right) \rightarrow D_{\mathbb{U}}(\mathcal{A})$. Then $\mathcal{L}$ is a pretriangulated category, so that $H^{0}(\mathcal{L}) \simeq L$. Hence also $D_{\mathbb{U}}(\mathcal{A}) / L \simeq H^{0}\left(\mathcal{S} \mathcal{F}_{\mathbb{U}}^{\text {str }}(\mathcal{A})\right) / H^{0}(\mathcal{L})$. Since the DG category $\mathcal{S} \mathcal{F}_{\mathbb{U}}^{\text {str }}(\mathcal{A})$ is $\mathbb{V}$-small the Drinfeld DG quotient $\mathcal{S} \mathcal{F}_{\mathbb{U}}^{\text {str }}(\mathcal{A}) / \mathcal{L}$ is defined, and by Theorem 1.3

$$
H^{0}\left(\mathcal{S} \mathcal{F}_{\mathbb{U}}^{\mathrm{str}}(\mathcal{A}) / \mathcal{L}\right) \simeq H^{0}\left(\mathcal{S} \mathcal{F}_{\mathbb{U}}^{\mathrm{str}}(\mathcal{A})\right) / H^{0}(\mathcal{L})
$$


Therefore $\mathcal{S} \mathcal{F}_{\mathbb{U}}^{\text {str }}(\mathcal{A}) / \mathcal{L}$ is a canonical enhancement of $D_{\mathbb{U}}(\mathcal{A}) / L$.

Lemma A.7. Let $\mathcal{A}$ be a $\mathbb{U}$-small $D G \mathbb{U}$-category. Then the derived category $D_{\mathbb{U}}(\mathcal{A})$ coincides with its full subcategory $\left\langle h^{\bullet}(\mathcal{A})\right\rangle^{\mathbb{U}}$.

Proof. Clearly the $\mathbb{U}$-small set $h^{\bullet}(\mathcal{A})$ of compact objects in $D_{\mathbb{U}}(\mathcal{A})$ generates the $\mathbb{U}$-cocomplete triangulated $\mathbb{U}$-category $D_{\mathbb{U}}(\mathcal{A})$. So it remains to apply part b) of Theorem A.1.

Proposition A.8. Let $\mathcal{C}$ be a pretriangulated $D G \mathbb{U}$-category. Assume that the triangulated $\mathbb{U}$-category $H^{0}(\mathcal{C})$ is $\mathbb{U}$-cocomplete and is generated by a $\mathbb{U}$-small set of compact objects $\mathcal{A} \subset H^{0}(\mathcal{C})^{c}$. Consider $\mathcal{A}$ as a full $D G$ subcategory in $\mathcal{C}$. The $D G$ functor $\Phi: \mathcal{C} \rightarrow \mathcal{M o d}_{\mathbb{U}}-\mathcal{A}$ defined as

$$
\Phi(X)(Y)=\operatorname{Hom}_{\mathcal{B}}(Y, X)
$$

for $X \in \mathcal{C}$ and $Y \in \mathcal{A}$ induces a quasi-functor $\phi: \mathcal{C} \rightarrow \mathcal{S F}(\mathcal{A})$ such that the functor $H^{0}(\phi): H^{0}(\mathcal{B}) \rightarrow D_{\mathbb{U}}(\mathcal{A})$ is an equivalence of categories.

Proof. First one shows (as in the proof of Proposition 1.19) that $H^{0}(\phi)$ preserves direct sums.

By the Brown representability theorem part b) one knows that $H^{0}(\mathcal{B})=\langle\mathcal{A}\rangle^{\mathbb{U}}$. Hence the same proof as in Proposition 1.17 shows that $H^{0}(\phi)$ is full and faithful.

On the other hand, the essential image of $H^{0}(\phi)$ is a full trianglated subcategory of $D_{\mathbb{U}}(\mathcal{A})$ closed under $\mathbb{U}$-small direct sums and containing the $\mathbb{U}$-small set $h^{\bullet}(\mathcal{A})$ of compact generators. Thus $H^{0}(\phi)$ is essentially surjective by Lemma A.7 above.

The following proposition shows that things don't change much when we pass to a larger universe or even consider the whole category of DG modules.

Proposition A.9. Let $\mathcal{A}$ be a $\mathbb{U}$-small $D G \mathbb{U}$-category. Then

(1) The category $D_{\mathbb{U}}(\mathcal{A})$ is a full subcategory of $D(\mathcal{A})$.

(2) Let $L \subset D(\mathcal{A})$ be a localizing subcategory generated by objects which are in $L_{\mathbb{U}}:=L \cap D_{\mathbb{U}}(\mathcal{A})$. (For example, $L$ may be compactly generated.) Then the natural functor $D_{\mathbb{U}}(\mathcal{A}) / L_{\mathbb{U}} \rightarrow D(\mathcal{A}) / L$ is full and faithful.

Proof. (1) Consider the diagram $M \stackrel{f}{\leftarrow} P \stackrel{s}{\rightarrow} N$ in $H^{0}(\mathcal{M}$ od $-\mathcal{A})$, where $M, N \in$ $H^{0}\left(\mathcal{M o d}_{\mathbb{U}}-\mathcal{A}\right)$ and $s$ is a quasi-isomorphism. It suffices to prove that there exists $Q \in H^{0}\left(\mathcal{M o d}_{\mathbb{U}}-\mathcal{A}\right)$ and a morphism $g: Q \rightarrow P$, such that $s \cdot g$ is a quasiisomorphism. Take $Q \in \mathcal{S F}_{\mathbb{U}}(\mathcal{A})$ and a quasi-isomorphism $t: Q \rightarrow N$. Then there exists $g: Q \rightarrow P$, such that $t=s \cdot g$. This proves $(1)$.

(2) Following [N3] we denote by $D(\mathcal{A})^{(\mathbb{U})} \subset D(\mathcal{A})$ the full triangulated subcategory of all objects $K$ such that for any collection $\left\{X_{\lambda}, \lambda \in \Lambda\right\}$ of objects of $D(\mathcal{A})$ any map $K \rightarrow \bigoplus_{\lambda \in \Lambda} X_{\lambda}$ factors through a direct subsum of cardinality strictly less than $\mathbb{U}$. (These are called $\mathbb{U}$-small in $\mathbb{N} 3$, but we already use this term in a different way.) Also denote by

$$
\left\{D(\mathcal{A})^{(\mathbb{U})}\right\}_{\mathbb{U}} \subset D(\mathcal{A})^{(\mathbb{U})}
$$

the full triangulated subcategory of $\mathbb{U}$-perfect objects (see Defs. 3.3.1 and 4.2.2 in N3]). This category $\left\{D(\mathcal{A})^{(\mathbb{U})}\right\}_{\mathbb{U}}$ is denoted by $D(\mathcal{A})^{\mathbb{U}}$ and its objects are called $\mathbb{U}$-compact objects of $D(\mathcal{A})$. (The categories $D(\mathcal{A})^{(\mathbb{U})}$ and $D(\mathcal{A})^{\mathbb{U}}$ are indeed triangulated, because $\mathbb{U}$ is an infinite cardinal (Lemmas 4.1.4 and Corollary 3.3.12 in [N3]).) Since $\mathbb{U}$ is a regular cardinal these categories are also $\mathbb{U}$-cocomplete (Lemma 
4.1.5 and Corollary 3.3.14 in [N3]). It follows that $D_{\mathbb{U}}(\mathcal{A}) \subseteq D(\mathcal{A})^{\mathbb{U}}$. Moreover, it is proved in Lemma 4.4.5 [N3] that in fact $D_{\mathbb{U}}(\mathcal{A})=D(\mathcal{A})^{\mathbb{U}}$.

Notice that $L_{\mathbb{U}}=\left\langle L_{\mathbb{U}}\right\rangle^{\mathbb{U}}$, since $L$ is cocomplete and $D_{\mathbb{U}}(\mathcal{A})$ is $\mathbb{U}$-cocomplete. Now Corollary 4.4.1 in [N3] asserts that the functor $D_{\mathbb{U}}(\mathcal{A}) / L_{\mathbb{U}} \rightarrow D(\mathcal{A}) / L$ is full and faithful.

\section{Appendix B. Proof of Proposition 9.6}

In this appendix we present a proof of Proposition 9.6. It is essentially the same as the proof of Proposition 9.3 that is given in $\mathrm{O} 1, \mathrm{O} 2$. We will directly follow O2, 3.4.6] and will use the notation of that proof.

Let $\mathfrak{E}$ be an exact category. Assume that it is a full exact subcategory of an abelian category $\mathfrak{A}$; i.e., $\mathfrak{E} \subset \mathfrak{A}$ is closed under extensions in $\mathfrak{A}$. We also assume that the additional property (EPI) holds:

(EPI) a map $f$ in $\mathfrak{E}$ is an admissible epimorphism if and only if it is an epimorphism in $\mathfrak{A}$.

As in Definition 9.4 we say that the sequence $\left\{P_{i} \mid i \in \mathbb{Z}\right\}$ of objects in $\mathfrak{E}$ is ample in $\mathfrak{E}$ if it is ample in $\mathfrak{A}$ as in Definition 9.1

Let us consider the derived categories $D^{*}(\mathfrak{E})$. Since $\mathfrak{E} \subset \mathfrak{A}$ is an exact subcategory of an abelian category $\mathfrak{A}$ such that the condition (EPI) holds and for any $C \in \mathfrak{A}$ there is an epimorphism $E \rightarrow C$ from $E \in \mathfrak{E}$, then the canonical functor $D^{-}(\mathfrak{E}) \rightarrow$ $D^{-}(\mathfrak{A})$ is an equivalence and the functor $D^{b}(\mathfrak{E}) \rightarrow D^{b}(\mathfrak{A})$ is fully faithful $\mathrm{K} 2$.

Proposition B.1. Let $\mathfrak{E}$ be an exact category with an ample sequence $\left\{P_{i} \mid i \in\right.$ $\left.\mathbb{Z}_{<0}\right\}$. Let $j: \mathcal{P} \hookrightarrow D^{b}(\mathfrak{E})$ be the natural embedding of the full subcategory $\mathcal{P}$ with objects $\mathrm{Ob} \mathcal{P}:=\left\{P_{i} \mid i \in \mathbb{Z}_{\leq 0}\right\}$. Let $F: D^{b}(\mathfrak{E}) \stackrel{\sim}{\rightarrow} D^{b}(\mathfrak{E})$ be an autoequivalence. Suppose that there is an isomorphism of functors $f:\left.j \stackrel{\sim}{\rightarrow} F\right|_{\mathcal{P}}$. Then $f$ can be extended to an isomorphism id $\stackrel{\sim}{\rightarrow} F$ on the whole category $D^{b}(\mathfrak{E})$.

Proof. A proof of this proposition is essentially the same as the proof of Proposition 9.3. We consider the canonical functor from $D^{b}(\mathfrak{E}) \rightarrow D^{b}(\mathfrak{A})$. It is fully faithful, because any object $C \in \mathfrak{A}$ can be covered by a direct sum of $P_{i}$ that belongs to $\mathfrak{E}$. This means that we can work with $D^{b}(\mathfrak{E})$ as a full triangulated subcategory of $D^{b}(\mathfrak{A})$ and, in particular, we can talk about cohomologies of a complex from $D^{b}(\mathfrak{E})$ as objects of $\mathfrak{A}$.

First, since $F$ commutes with finite direct sums, the transformation $f$ extends componentwise to all finite direct sums of objects of the category $\mathcal{P}$. Note that an object $X \in D^{b}(\mathfrak{E})$ is isomorphic to an object of $\mathfrak{A}$ if and only if $\operatorname{Hom}^{j}\left(P_{i}, X\right)=0$ for all $j \neq 0$ when $i \ll 0$. It follows that in this case the object $F(X)$ is also isomorphic to an object of $\mathcal{A}$, because

$$
\operatorname{Hom}^{j}\left(P_{i}, F(X)\right) \cong \operatorname{Hom}^{j}\left(F\left(P_{i}\right), F(X)\right) \cong \operatorname{Hom}^{j}\left(P_{i}, X\right)=0
$$

for $j \neq 0$ when $i \ll 0$.

Step 1. In Step 1 we construct an isomorphism $f_{X}: X \stackrel{\sim}{\rightarrow} F(X)$ for all $X \in D^{b}(\mathfrak{E})$ that are isomorphic to an object of $\mathfrak{A}$. Let $X$ be such an object. Then $X \cong H^{0}(X)$. We fix a morphism $v: P_{i}^{\oplus k} \rightarrow X$ such that the canonical map $P_{i}^{\oplus k} \rightarrow H^{0}(X)$ is surjective in the abelian category $\mathfrak{A}$. After that the proof of Step 1 is the same as the proof of Step 1 in $[\mathrm{O} 2,3.4 .6]$. 
Step 2. Now we show that $f_{X}$ does not depend on the choice of the morphism $v: P_{i}^{\oplus k} \rightarrow X$. The proof of Step 2 is the same as the proof of Step 2 in [O2, 3.4.6].

Step 3. In Step 3 we check that the morphisms $f_{X}$ define a natural transformation of functors on the subcategory of $D^{b}(\mathfrak{E})$ consisting of all objects that are isomorphic to objects of the abelian category $\mathfrak{A}$. That is, for any morphism of such objects $\varphi: X \rightarrow Y$, we have to prove that $f_{Y} \cdot \varphi=F(\varphi) \cdot f_{X}$. The proof of this step is word for word the same as the proof of Step 3 in [O2, 3.4.6].

Step 4. We constructed transformations $f_{X}: X \rightarrow F(X)$ for all $X \in D^{b}(\mathfrak{E})$ that are isomorphic to objects of the abelian category $\mathfrak{A}$. Now we define $f_{X[n]}: X[n] \rightarrow$ $F(X[n]) \cong F(X)[n]$ for any such $X$ by the evident formula $f_{X[n]}=f_{X}[n]$. We need to show that these transformations commute with any $u \in \operatorname{Hom}(X, Y[k])$ for all $k>0$. For an abelian category we used the fact that any element $u \in$ $\operatorname{Hom}(X, Y[k])$ can be represented as a composition $u=u_{k} \cdots u_{1}$ of some elements $u_{i} \in \operatorname{Hom}\left(Z_{i-1}, Z_{i}[1]\right)$ with $Z_{0}=X, Z_{k}=Y$. That allowed us to reduce the problem to the case $u \in \operatorname{Hom}(X, Y[1])$. In the case of $D^{b}(\mathfrak{E})$ we should correct the argument.

Let $X$ and $Y$ be objects of $D^{b}(\mathfrak{E})$ that are isomorphic to objects of the abelian category $\mathfrak{A}$. A fiber of a morphism $u \in \operatorname{Hom}(X, Y[k])$ when $k \geq 2$ can be represented by a complex over $\mathfrak{A}$ of the form $C^{\cdot}: C^{-k+1} \rightarrow \cdots \rightarrow C^{0}$ which has only two nontrivial comomologies $H^{0}\left(C^{\cdot}\right) \cong X$ and $H^{-k+1}\left(C^{\cdot}\right) \cong Y$. By Remark 9.5 there are a complex $E^{\cdot}:\left\{\cdots \stackrel{d^{-2}}{\longrightarrow} E^{-1} \stackrel{d^{-1}}{\longrightarrow} E^{0}\right\}$ over $\mathfrak{E}$ and a quasi-isomorphism $E^{\cdot} \rightarrow C^{\cdot}$. Consider the usual truncation of the form $\tau_{\geq-k+1} E^{\cdot}:\left\{\right.$ Coker $d^{-k} \rightarrow E^{-k+2} \rightarrow$ $\left.\cdots \rightarrow E^{0}\right\}$ and the induced quasi-isomorphism $\tau_{\geq-k+1} E^{\cdot} \rightarrow C$. It is easy to see that the object $\operatorname{Im} d^{-1}$ as a fiber of the map $E^{0} \rightarrow X$ is isomorphic to an object of $D^{b}(\mathfrak{E})$. Similarly, each $\operatorname{Im} d^{-i}=\operatorname{Ker} d^{-i+1}$ for $2 \leq i \leq k-1$ is also isomorphic to an object of $D^{b}(\mathfrak{E})$. Finally, Coker $d^{-k}$ as an extension of $\operatorname{Im} d^{-k+1}$ and $Y$ is also isomorphic to an object of $D^{b}(\mathfrak{E})$. Now we put $Z_{0}=X, Z_{k}=Y$ and $Z_{i}=\operatorname{Im} d^{-i}$ for $i=1, \ldots, k-1$ with corresponding $u_{i} \in \operatorname{Hom}\left(Z_{i-1}, Z_{i}[1]\right)$. Thus, the morphism $u$ can be represented as a composition $u=u_{k} \cdots u_{1}$. Therefore, it is sufficient to verify that $f_{X[n]}$ commutes with elements $u \in \operatorname{Hom}(X, Y[1])$.

The rest of the proof is the same as the corresponding part of the proof of Step 4 in $\mathrm{O} 2,3.4 .6$.$] .$

Step 5. We carry out the final part of the proof by induction on the length of the interval to which the nontrivial cohomology of the object belongs. For this consider the full subcategory $j_{n}: \mathcal{D}_{n} \hookrightarrow D^{b}(\mathfrak{E})$ consisting of objects with nontrivial cohomologies in some interval of length $n$ (the interval is not fixed). We now prove that there is a unique extension of the natural transformation $f$ to a natural functorial isomorphism $f_{n}:\left.j_{n} \rightarrow F\right|_{\mathcal{D}_{n}}$. We have already proved this above for $n=1$, as the basis of the induction.

Now to prove the induction step, suppose that the assertion is already proved for some $n=a \geq 1$. Let $X$ be an object of $\mathcal{D}_{a+1}$. In Step 5 we construct an isomorphism $f_{X}: X \rightarrow F(X)$ for all $X \in \mathcal{D}_{a+1}$. The proof is the same as the proof of Step 5 in $[\mathrm{O} 2,3.4 .6]$.

Step 6. Now we have to prove that the isomorphism $f_{X}$ does not depend on the choices made in the construction in Step 5. The proof is word for word as the proof of Step 6 in [O2, 3.4.6]. 
Step 7. We constructed isomorphisms $f_{X}: X \rightarrow F(X)$ for all $X \in \mathcal{D}_{a+1}$. It remains to show that this extension of $f_{a}$ is a natural transformation from $j_{a+1}$ to $\left.F\right|_{\mathcal{D}_{a+1}}$. Thus, we have to check that for any $\varphi: X \rightarrow Y$ with $X$ and $Y$ in $\mathcal{D}_{a+1}$ we obtain a commutative diagram

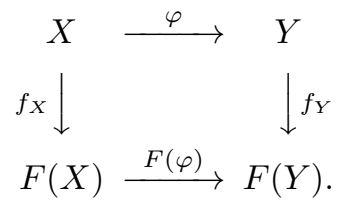

We reduce this problem to the case in which both objects $X$ and $Y$ belong to $\mathcal{D}_{a}$. The proof is the same as the proof of Step 7 in [02, 3.4.6].

\section{ACKNOWLEDGMENTS}

The main results of this paper were reported by the first author in December 2008 at the conference on triangulated categories at Swansea University. Recently some results on representability of fully faithful functors between categories of perfect complexes were independently obtained in $\mathrm{Ba}$.

The second author is grateful to C. Lazaroiu and A. Kuznetsov for very useful discussions. We thank the anonymous referee for a careful reading of the text, making several useful suggestions and for finding a few minor errors in the original version.

\section{REFERENCES}

[SGA4] M. Artin, A. Grothendieck, J. L. Verdier, Théorie des topos et cohomologie étale des schémas. Tome 1: Théorie des topos. (French) Séminaire de Géométrie Algébrique du Bois-Marie 1963-1964 (SGA 4). Lect. Notes in Math., 269 (1972). MR0354652 (50:7130)

[Ba] M. R. Ballard, Equivalences of derived categories of sheaves on quasi-projective schemes, arXiv:0905.3148.

[BN] M. Bökstedt, A. Neeman, Homotopy limits in triangulated categories, Comp. Math., 86 (1993), 2, 209-234. MR.1214458 (94f:18008)

[BvB] A. Bondal, M. Van den Bergh, Generators and representability of functors in commutative and noncommutative geometry, Mosc. Math. J., 3 (2003), 1-36. MR.1996800 (2004h:18009)

[BK] A. Bondal, M. Kapranov, Enhanced triangulated categories, Math. USSR-Sbornik, 70 (1991), 1, 93-107. MR1055981(91g:18010)

[BLL] A. Bondal, M. Larsen, V. Lunts, Grothendieck ring of pretriangulated categories, Int. Math. Res. Not., 29 (2004), 1461-1495. MR2051435 (2005d:18014)

[Dr] V. Drinfeld, DG quotients of DG categories, J. of Algebra, 272 (2004), 5, 643-691. MR 2028075 (2006e:18018)

[ELO1] A. Efimov, V. Lunts, D. Orlov, Deformation theory of objects in homotopy and derived categories I: General theory, Adv. Math., 222 (2009), 2, 359-401. MR2538013

[Ga] P. Gabriel, Des catégories abéliennes, Bull. de la Soc. Math. de France, 90 (1962), 323448. MR0232821 (38:1144)

[EGA1] A. Grothendieck, J. Dieudonné, Éléments de géométrie algébrique, Grundleheren, 166 (1971), Press Univ. France, Springer-Verlag. MR0432634 (55:5621)

[EGA2] A. Grothendieck, Éléments de géométrie algébrique (rédigés avec la collaboration de Jean Dieudonné): II. Étude globale élémentaire de quelques classes de morphismes, Publ. Math. de l'IHÉS, 8 (1961), 5-222. MR0217084(36:177b)

[Ha] R. Hartshorne, Residues and Duality, Lect. Notes Math., 20 (1966). MR0222093 $(36: 5145)$

[Hi] V. Hinich, Homological algebra of homotopy algebras, Comm. Algebra, 25 (1997), 10, 3291-3323. MR1465117 (99b:18017) 
[IENT] F. Castaño Iglesias, P. Enache, C. Năstăsescu, B. Torrecillas, Gabriel-Popescu type theorems and applications, Bull. Sci. Math., 128 (2004), n.4, 323-332. MR 2052174 (2005e:18015)

[KS] M. Kashiwara, P. Shapira, Categories and sheaves, Springer-Verlag (2006). MR2182076 (2006k:18001)

[K1] B. Keller, Deriving DG categories, Ann. Sci. de l'École Norm. Sup., Sér. 4, 27 (1994), 63-102. MR.1258406 (95e:18010)

[K2] B. Keller, Derived categories and their uses, Handbook of algebra, 1, North-Holland, Amsterdam, (1996), 671-701. MR1421815 (98h:18013)

[Li] J. Lipman, Lectures on local cohomology and duality, In "Local Cohomology and Its Applications," Lect. Notes in Pure and Applied Math., 226, Marcel Dekker, NY, (2001), 39-89. MR.1888195 (2003b:13027)

[Me] C. Menini, Gabriel-Popescu type theorems and graded modules, Perspectives in ring theory (Antwerp, 1987), 239-251, NATO Adv. Sci. Inst. Ser. C, Math. Phys. Sci., 233, Kluwer Acad. Publ., Dordrecht, (1988). MR1048412 (91d:18011)

[N1] A. Neeman, The connection between the K-theory localization theorem of Thomason, Trobaugh and Yao and the smashing subcategories of Bousfield and Ravenel, Ann. Sci. de l'École Norm. Sup., Sér. 4, 25 (1992), 5, 547-566. MR1191736 (93k:18015)

[N2] A. Neeman, The Grothendieck duality theorem via Bousfield's techniques and Brown representability, J. Amer. Math. Soc., 9 (1996), 205-236. MR.1308405 (96c:18006)

[N3] A. Neeman, Triangulated categories, Ann. of Math. Studies, 148, Princeton Univ. Press, (2001). MR.1812507 (2001k:18010)

[OSS] C. Okonek, M. Schneider, H. Spindler, Vector bundles on complex projective spaces, Progress in Math., 3. Birkhäuser, Boston, Mass., (1980). MR561910 (81b:14001)

[O1] D. Orlov, Equivalences of derived categories and K3 surfaces, J. Math. Sci., 84 (1997), 5, 1361-1381. MR 1465519 (99a:14054)

[O2] D. Orlov, Derived categories of coherent sheaves and equivalences between them, Russian Math. Surveys, 58 (2003), 3, 89-172. MR1998775 (2004g:14021)

[Po] N. Popescu, Abelian categories with Applications to Rings and Modules, Academic Press, L. M. S. Monograph No.3, London, (1973). MR0340375 (49:5130)

[Se] J.-P. Serre, Faisceaux algébriques cohérents, Ann. of Math., 2nd Ser., 61 (1955), 2, 197278. MR0068874 (16:953c)

[Sp] N. Spaltenstein, Resolution of unbounded complexes, Comp. Math., 65 (1988), 2, 121-154. MR.932640 (89m:18013)

[Ta] G. Tabuada, Théorie homotopique des DG-catégories, Thèse de l'Univ. Paris 7 (2007).

[To] B. Toën, The homotopy theory of dg-categories and derived Morita theory, Invent. Math., 167 (2007), 3, 615-667. MR2276263 (2008a:18006)

[TT] R. W. Thomason, T. Trobaugh, Higher Algebraic K-Theory of Schemes and of Derived Categories, The Grothendieck Festschrift III, Birhäuser, Boston, Basel, Berlin, (1990), 247-436. MR1106918 (92f:19001)

[Th] R. W. Thomason, The classification of triangulated subcategories, Compositio Math., 105 (1997), 1, 1-27. MR.1436741 (98b:18017)

Department of Mathematics, Indiana University, Bloomington, Indiana 47405

E-mail address: vlunts@indiana.edu

Steklov Mathematical Institute, 8 Gubkina St., Moscow, Russia

E-mail address: orlov@mi.ras.ru 